\title{
Quantum theory in quaternionic Hilbert space: How Poincaré symmetry reduces the theory to the standard complex one.
}

\author{
Valter Moretti ${ }^{a}$, Marco Oppio ${ }^{b}$ \\ ${ }^{a}$ Department of Mathematics University of Trento, and INFN-TIFPA \\ via Sommarive 15, I-38123 Povo (Trento), Italy. \\ valter.moretti@unitn.it \\ ${ }^{b}$ Faculty of Mathematics, University of Regensburg, \\ Universitätstrasse 31, 93053 Regensburg, Germany \\ marco.oppio@ur.de
}

November 06, 2018

\begin{abstract}
As earlier conjectured by several authors and much later established by Solèr, from the lattice-theory point of view Quantum Mechanics may be formulated in real, complex or quaternionic Hilbert spaces only. On the other hand no quantum systems seem to exist that are naturally described in a real or quaternionic Hilbert space. In a previous paper [23, we showed that any quantum system which is elementary from the viewpoint of the Poincare symmetry group and it is initially described in a real Hilbert space, it can also be described within the standard complex-Hilbert space framework. This complex description is unique and more precise than the real one as for instance, in the complex description, all self-adjoint operators represent observables defined by the symmetry group. The complex picture fulfils the thesis of Solér theorem and permits the standard formulation of quantum Noether's theorem. The present work is devoted to investigate the remaining case, namely the possibility of a description of a relativistic elementary quantum system in a quaternionic Hilbert space. Everything is done exploiting recent results of quaternionic spectral theory independently developed. In the initial part of this work, we extend some results of group representation theory and von Neumann algebra theory from the real and complex case to the quaternionic Hilbert space case. We prove the double commutant theorem also for quaternionic von Neumann algebras (whose proof requires a different procedure with respect to the real and complex cases) and we extend to the quaternionic case a result established in the previous paper concerning the classification of irreducible von Neumann algebras into three categories. In the second part of the paper, we consider an elementary relativistic system within Wigner's approach defined as a locally-faithful irreducible strongly-continuous unitary representation of the Poincaré group in a quaternionic Hilbert space. We prove that, if the squared-mass operator is non-negative, the system admits a natural, Poincaré invariant and unique up to sign, complex structure which commutes with the whole algebra of observables generated by the representation itself. This complex structure leads to a physically equivalent reformulation of the theory in a complex Hilbert space. Within this complex formulation, differently from what happens in the quaternionic one, all selfadjoint operators represent observables in agreement with Solèr's thesis, the standard quantum version of Noether theorem may be formulated and the notion of composite system may be given in terms of tensor product of elementary systems. In the third part of the paper, we focus on the
\end{abstract}


physical hypotheses adopted to define a quantum elementary relativistic system relaxing them on the one hand, and making our model physically more general on the other hand. We use a physically more accurate notion of irreducibility regarding the algebra of observables only, we describe the symmetries in terms of automorphisms of the restricted lattice of elementary propositions of the quantum system and we adopt a notion of continuity referred to the states viewed as probability measures on the elementary propositions. Also in this case, the final result proves that there exist a unique (up to sign) Poincaré invariant complex structure making the theory complex and completely fitting into Solèr's picture. The overall conclusion is that relativistic elementary systems are naturally and better described in complex Hilbert spaces even if starting from a real or quaternionic Hilbert space formulation and this complex description is uniquely fixed by physics. 


\section{Contents}

1 Introduction 4

1.1 Quaternionic Hilbert spaces . . . . . . . . . . . . . . . . . 4

1.2 The lattice of elementary propositions of a quantum system . . . . . . . 5

1.3 The coordinatisation problem ................ 6

1.4 Theoretical notions in common with the three types of Hilbert spaces formulations . . . . . . . . . . . . . . . . 8

1.5 Main results of this work . . . . . . . . . . . . . . . . 11

2 Quaternionic operators and their natural structures $\quad 12$

2.1 von Neumann algebras in quaternionic Hilbert spaces . . . . . . . . . . . . 12

2.2 The lattice of orthogonal projectors of quaternionic von Neumann algebras 17

2.3 Stone theorem . . . . . . . . . . . . . . . . . . . 21

2.4 Polar decomposition of unbounded operators in quaternionic Hilbert spaces 22

3 Restriction to subspaces induced by complex structures $\quad 23$

3.1 Restriction to a complex Hilbert subspace induced by a complex structure 24

3.2 Restriction to a real Hilbert subspace induced by two anticommuting complex structures .................... . . . . 26

4 Lie-Group representations in quaternionic Hilbert spaces $\quad 29$

4.1 Gårding domain and Lie algebra representation . . . . . . . . . . . . . . . 29

4.2 Analytic vectors of unitary representations in quaternionic Hilbert spaces . 33

4.3 Group representations restricted to a complex Hilbert subspace induced by a complex structure . . . . . . . . . . . . . . . . 34

4.4 Group representations restricted to a real Hilbert subspace induced by two anti commuting complex structures . . . . . . . . . . . . . 35

5 Irreducibility and Schur's Lemma on Quaternionic Hilbert spaces $\quad 36$

5.1 Schur's lemma in quaternionic Hilbert spaces . . . . . . . . . . . . . . . . . 36

5.2 Application to Lie-group representations . . . . . . . . . . . . . . . . . 38

6 Irreducible quaternionic von Neumann algebras $\quad 40$

6.1 The commutant of an irreducible quaternionic von Neumann algebra . . . 40

6.2 The structure of irreducible von Neumann algebras in quaternionc Hilbert spaces. . . . . . . . . . . . . . . . . . 41

7 Quaternionic Wigner relativistic elementary systems (WRES) 43

7.1 Faithfulness issues . . . . . . . . . . . . . . . . . . 43

7.2 Wigner's approach extend to the quaternionic case . . . . . . . . . . . . 44

7.3 Structure of quaternionic a WRES and equivalence with a complex WRES 50

8 Quaternionic relativistic elementary systems (qRES) 53

8.1 Elementary systems . . . . . . . . . . . . . . . . . . 53

8.2 Relativistic elementary systems in quaternionic Hilbert spaces . . . . . . . 57

8.3 Reduction to the complex Hilbert space case . . . . . . . . . . . . . . . . . 60

9 Conclusions $\quad 62$

\begin{tabular}{l|l|} 
A Proof of some propositions & 63 \\
\hline
\end{tabular} 


\section{Introduction}

It is known that quantum theories can be formulated in real, complex and quanternionic Hilbert spaces as we summarize below. A brief account of basic real Hilbert space spectral theory appears in [23] and a summary on basic results on quaternionic Hilbert space theory is included in the next section, whereas for the general spectral theory of unbounded normal operators we address to [11, 13].

\subsection{Quaternionic Hilbert spaces}

$\mathbb{H}:=\{a 1+b i+c j+d k \mid a, b, c, d \in \mathbb{R}\}$ henceforth denotes the real unital associative algebra of quaternions. $i, j, k$ are the standard imaginary units satisfying $i^{2}=j^{2}=k^{2}=-1$ and $i j=-j i=k, j k=-k j=i, k i=-i k=j$ which give rise to the notion of associative, distributive and non-commutative product in $\mathbb{H}$ with 1 as neutral element. $\mathbb{H}$ is a division algebra, i.e., every non zero element admits a multiplicative inverse. The centre of $\mathbb{H}$ is $\mathbb{R}$. $\mathbb{H}$ is assumed to be equipped with the quaternionic conjugation $\overline{a 1+b i+c j+d k}=a 1-b i-c j-d k$. Notice that the conjugation satisfies $\overline{q q^{\prime}}=\overline{q^{\prime}} \bar{q}$ and $\overline{\bar{q}}=q$ for all $q, q^{\prime} \in \mathbb{H}$. If $q \in \mathbb{H}$, its real part is defined as $\operatorname{Re} q:=\frac{1}{2}(q+\bar{q}) \in \mathbb{R}$. The quaternionic conjugation together with the Euclidean norm $|q|:=\sqrt{q \bar{q}}$ for $q \in \mathbb{H}$, makes $\mathbb{H}$ a real unital $C^{*}$-algebra which also satisfies the composition algebra property $\left|q q^{\prime}\right|=|q|\left|q^{\prime}\right|$.

Definition 1.1 A quaternionic vector space is an additive Abelian group $(\mathrm{H},+)$ denoting the sum operation, equipped with a right-multiplication $\mathrm{H} \times \mathbb{H} \ni(x, q) \mapsto x q \in \mathrm{H}$ such that (a) the right-multiplication is distributive with respect to,+ , (b) the sum of quaternions is distributive with respect to the right-multiplication, (c) $(x q) q^{\prime}=x\left(q q^{\prime}\right)$ and (d) $x 1=x$ for all $x \in \mathrm{H}$ and $q, q^{\prime} \in \mathbb{H}$.

Definition 1.2 A quaternionic Hilbert space is a quaternionic vector space $\mathrm{H}$ equipped with a Hermitian quaternionic scalar product, i.e., a map $\mathrm{H} \times \mathrm{H} \ni(x, y) \mapsto$ $\langle x \mid y\rangle \in \mathbb{H}$ such that (a) $\langle x \mid y q+z\rangle=\langle x \mid y\rangle q+\langle x \mid z\rangle$ for every $x, y, z \in \mathbb{H}$ and $q \in \mathbb{H}$, (b) $\langle x \mid y\rangle=\overline{\langle y \mid x\rangle}$ for every $x, y \in \mathrm{H}$ and (c) $\langle x \mid x\rangle \in[0,+\infty)$ where (d) $\langle x \mid x\rangle=0$ implies $x=0$, and $\mathrm{H}$ is complete with respect to the norm $\|x\|=\sqrt{\langle x \mid x\rangle}$.

The standard Cauchy-Schwartz inequality holds, $|\langle x \mid y\rangle| \leq\|x\|\|y\|$ for every $x, y \in \mathrm{H}$ for the above defined quaternionic Hermitian scalar product [11. The notion of Hilbert basis is the same as for real and complex Hilbert spaces and properties are the same with obvious changes. A quaternionic Hilbert space turns out to be separable as a metrical space if and only if it admits a finite or countable Hilbert basis. The notion of orthogonal subspace $S^{\perp}$ of a set $S \subset \mathrm{H}$ is defined with respect to $\langle\cdot \cdot \cdot\rangle$ and enjoys the same standard properties as for the analogue in real and complex Hilbert spaces. The notion of operator norm and bounded operator are the same as for real and complex Hilbert spaces. Since the Riesz lemma [11] holds true also for quaternionic Hilbert spaces, the adjoint operator $A^{*}: \mathrm{H} \rightarrow \mathrm{H}$ of a bounded quaternionic linear operator $A: \mathrm{H} \rightarrow \mathrm{H}$ can be defined as the 
unique quaternionic linear operator such that $\left\langle A^{*} y \mid x\right\rangle=\langle y \mid A x\rangle$ for every pair $x, y \in \mathrm{H}$. Notice that if $A: \mathrm{H} \rightarrow \mathrm{H}$ is quaternionic linear and $r \in \mathbb{R}$, we can define the quaternionic linear operator $r A: \mathrm{H} \rightarrow \mathrm{H}$ such that $r A x:=(A x) r$ for all $x \in \mathrm{H}$. Replacing $r$ for $q \in \mathbb{H}$ produces a non-linear map in view of non-commutativity of $\mathbb{H}$. Therefore only real linear combinations of quaternionic linear operators are well defined. $\mathfrak{B}(\mathrm{H})$ denotes the real unital $C^{*}$-algebra of bounded operators over $\mathrm{H}$. The notion of orthogonal projector $P: \mathrm{H} \rightarrow \mathrm{H}$ is defined exactly as in the real or complex Hilbert space case, $P$ is bounded, $P P=P$ and $P^{*}=P$. Orthogonal projectors $P$ are one-to-one with the class of closed subspaces $P(\mathrm{H})$ of $\mathrm{H} . \mathcal{L}(\mathrm{H})$ denotes the orthocomplemented complete lattice (see below) of orthogonal projectors of $\mathrm{H}$. Another important notion is that of square root of positive bounded operators. As for the real and complex case, also for quaternionic Hilbert spaces, if $A$ is bounded and positive, then there exists a unique bounded positive operator $\sqrt{A}$ such that $\sqrt{A} \sqrt{A}=A[11$, 13]. In particular, if $A: \mathrm{H} \rightarrow \mathrm{H}$ is a bounded quaternioniclinear operator $|A|:=\sqrt{A^{*} A}$ is well defined positive and self-adjoint. For the proofs of the afore-mentioned properties and for more advanced issues, especially concerning spectral theory, we address the reader to [11] and [13].

Remark 1.3 In [40] and [8] the Quaternionic Hilbert space is defined assuming a leftmultiplication $\mathbb{H} \times \mathrm{H} \ni(q, u) \mapsto q u \in \mathrm{H}$ and a Hermitian quaternionic scalar product $\mathrm{H} \times \mathrm{H} \ni(u, v) \mapsto\langle u \mid v\rangle \in \mathbb{H}$ whose only difference resides in point (a): $\langle q x \mid y\rangle=q\langle x \mid y\rangle$ for all $x, y \in \mathrm{H}$ and $q \in \mathbb{H}$. To define a left-multiplication on a space with right-multiplication it suffices to define $q u:=u \bar{q}$ for all $q \in \mathbb{H}$ and $u \in \mathrm{H}$, while the scalar product does not need to be changed. A map $A: \mathrm{H} \rightarrow \mathrm{H}$ is linear, bounded, self-adjoint, idempotent and unitary with respect to the right-multiplication if and only if it has the same properties with respect to the left-multiplication. This permits us to exploit indifferently the results in [11, 13] and [40, [8].

\subsection{The lattice of elementary propositions of a quantum system}

Quantum theory can basically be formulated as a non-Boolean probability theory over the partially ordered set of elementary propositions $\mathcal{L}$ about the given physical quantum system [4, 40, 32]. Let us review some elementary ideas on this subject restating the discussion already present in the introduction of [23]. Elementary propositions, also called elementary observables, are the experimentally testable propositions with possible outcomes 0 and 1 . The partial order relation $\leq$ in $\mathcal{L}$ corresponds to the logical implication (see [19, 4, 40, 7] for the various interpretations). It is generally supposed that the partially ordered set $\mathcal{L}$ is a lattice (with some noticeable exception as [19]): A pair of elements $a, b \in \mathcal{L}$ always admits $\inf \{a, b\} \in \mathcal{L}=: a \wedge b$ called meet, and $\sup \{a, b\} \in=: a \vee b$ called join. It is easy to prove that $a \leq b$ if and only if $a=a \wedge b$ and that $\vee$ and $\wedge$ are separately symmetric and associative in every lattice. The $\mathcal{L}$ is also requested to be bounded: A minimal element $\mathbf{0}$, the always false proposition, and a maximal element $\mathbf{1}$, the always true proposition, exist in $\mathcal{L}$. $\mathcal{L}$ is also assumed to be $(\sigma$-)complete, i.e., $\sup A$ and $\inf A$ exist for every (countable) set $A \subset \mathcal{L}$. $\mathcal{L}$ is finally 
demanded to be orthocomplemented: If $a \in \mathcal{L}$, an orthogonal complement $a^{\perp} \in \mathcal{L}$ exists interpreted as the logical negation of $a$. By definition the orthocomplement satisfy $a \vee a^{\perp}=\mathbf{1}, a \wedge a^{\perp}=\mathbf{0},\left(a^{\perp}\right)^{\perp}=a$, and $a \leq b$ implies $b^{\perp} \leq a^{\perp}$ for any $a, b \in \mathcal{L}$. Now $a, b \in \mathcal{L}$ are orthogonal, written $a \perp b$, if $a \leq b^{\perp}$ (equivalently $b \leq a^{\perp}$ ).

Pairs of mutually compatible elementary propositions on a quantum system (those which are simultaneously testable by means of experiment) are described by pairs of commuting elements $p, q \in \mathcal{L}$ in the sense of abstract orthocomplemented lattices [4]: the sublattice generated by $\{p, q\}$, namely the intersection of all orthocomplemented sublattices of $\mathcal{L}$ which include $\{p, q\}$ is Boolean, i.e., $\vee$ and $\wedge$ are mutually distributive. A maximal set of pairwise compatible propositions is a complete Boolean sublattice and an interpretation in terms of classical logic is appropriate. Compatibility is not transitive and so the structure of maximal Boolean sublattices of $\mathcal{L}$ is very complicated. The whole lattice $\mathcal{L}$ of elementary propositions of a quantum system is however non-Boolean because $\wedge$ and $\vee$ are not mutually distributive. Physically speaking this is due to the existence of incompatible elementary propositions (e.g., see [4, 22]). The quantum lattice $\mathcal{L}$ enjoys a set of peculiar properties that can be phenomenologically motivated (e.g., see [4]) even if some non-trivial interpretative problems remain [7]: (i) orthomodularity, (ii) $\sigma$-completeness, (iii) atomicity, (iii)' atomisticity, (iv) covering property, (v) separability, (vi) irreducibility (see the appendix of [23] for a brief illustration of these definitions).

\subsection{The coordinatisation problem}

As it will be useful below and in the rest of the paper, we remind the reader that if $\mathcal{L}_{1}$, $\mathcal{L}_{2}$ are orthocomplemented lattices, a map $h: \mathcal{L}_{1} \rightarrow \mathcal{L}_{2}$ is a lattice homomorphism if $h\left(a \vee_{1} b\right)=h(a) \vee_{2} h(b), h\left(a \wedge_{1} b\right)=h(a) \wedge_{2} h(b), h(a)^{\perp_{2}}=h\left(a^{\perp_{1}}\right)$ if $a, b \in \mathcal{L}_{1}, h\left(\mathbf{0}_{1}\right)=\mathbf{0}_{2}$, $h\left(\mathbf{1}_{1}\right)=\mathbf{1}_{2}$. When the lattices are complete, resp. $\sigma$-complete, the first pair of conditions are made stronger to $h\left(\sup _{a \in A} a\right)=\sup _{a \in A} h(a)$ and $h\left(\inf _{a \in A} a\right)=\inf _{a \in A} h(a)$ for every infinite, resp. countably infinite, subset $A \subset \mathcal{L}_{1}$. A straightforward calculation shows that $a \leq_{1} b$ implies $h(a) \leq_{2} h(b)$. A bijective lattice homomorphism is a lattice isomorphism. The inverse map of a lattice isomorphism is a lattice isomorphism as well. Lattice automorphisms are isomorphisms with $\mathcal{L}_{1}=\mathcal{L}_{2}$; they form a group, denoted by $\operatorname{Aut}\left(\mathcal{L}_{1}\right)$.

The long standing coordinatisation problem [4] consisted of proving (or disproving) that an abstract bounded orthocomplemented lattice $\mathcal{L}$ satisfying (i)-(vi) and possibly some added technical requirements, is necessarily isomorphic to the lattice $\mathcal{L}(\mathrm{H})$ of the orthogonal projectors/closed subspaces of a complex Hilbert space $\mathrm{H}$. Here the partial order relation is the inclusion of the projection closed subaspace of the considered orthogonal projectors. This should provide a justification of the standard Hilbert-space formulation of Quantum Theory. Some intermediate and fundamental results by Piron [29] and Maeda-Maeda [20] established that such $\mathcal{L}$, if contains four orthogonal atoms at least, is necessarily isomorphic to the lattice of the orthoclosed subspaces $\left(K=K^{\perp \perp}\right)$ of a struc- 
ture generalizing a vector space over a division ring $\mathbb{D}$ equipped with a suitable involution operation, and admitting a generalized non-singular $\mathbb{D}$-valued Hermitian scalar product (giving rise to the above mentioned notion of orthogonal ${ }^{\perp}$ ). The order relation of this concrete lattice is the standard inclusion of orthoclosed subspaces. In 1995 Solèr [37] achieved the perhaps conclusive result (for alternative equivalent statements see [14] and $[2])$.

[Solér's Theorem]. Consider an orthocomplemented lattice $\mathcal{L}$ satisfying (i)-(vi), such that (vii) it contains at least four orthogonal atoms and (viii) $\mathcal{L}$ includes an infinite orthogonal sets of atoms with unit (generalized) norm. Then $\mathcal{L}$ is isomorphic to the lattice $\mathcal{L}(\mathrm{H})$ of (topologically) closed subspaces of a separable Hilbert space $\mathrm{H}$ with set of scalars given by either the fields $\mathbb{R}, \mathbb{C}$ or the real division algebra of quaternions $\mathbb{H}$.

In all three cases, the partial order relation of the lattice is again the standard inclusion of closed subspaces and $\mathrm{M} \vee \mathrm{N}$ corresponds to the closed span of the union of the closed subspaces $\mathrm{M}$ and $\mathrm{N}$, whereas $\mathrm{M} \wedge \mathrm{N}:=\mathrm{M} \cap \mathrm{N}$. The minimal element is the trivial subspace $\{0\}$ and the maximal element is $H$ itself. Finally, the orthocomplement of $M \in \mathcal{L}(H)$ is described by the standard orthogonal $\mathrm{M}^{\perp}$ in $\mathrm{H}$. All the structure can equivalently be rephrased in terms of orthogonal projectors $P$ in $\mathrm{H}$, since they are one-to-one associated with the closed subspaces of $\mathrm{H}$ identified with their images $P(\mathrm{H})$. In particular $P \leq Q$ corresponds to $P(\mathrm{H}) \subset Q(\mathrm{H})$ for $P, Q \in \mathcal{L}(\mathrm{H})$.

Dropping irreducibility requirement of $\mathcal{L}$ in Solèr's theorem, physically corresponding to absence of superselection rules, an orthogonal direct sum of many such Hilbert spaces (even over different set of scalars) replaces the single Hilbert space $\mathrm{H}$.

Solèr's theorem assumes a list of rigid requirements on the structure of $\mathcal{L}$ and the thesis represents an equally rigid picture. An evident physical lack in the hypotheses of the theorem is the absence of any fundamental physical symmetry requirement, according to Galileo or Poincaré groups. In complex Hilbert spaces, only type- $I$ factors are permitted by the thesis of Soler's theorem to represent the algebra of observables $\mathfrak{R}$ and no gauge group may enter the game therefore excluding systems of quarks where internal symmetries (colour $S U(3)$ ) play a crucial rôle. Solèr's picture is evidently not appropriate also to describe non-elementary quantum systems like pure phases of extended quantum thermodynamic systems. There the algebra of observables is still a factor, but the type- $I$ is not admitted in general due to the presence of a non-trivial commutant $\mathfrak{R}$. Also localized algebras of observables in QFT are not encompassed by Solèr's framework. However, elementary relativistic systems like elementary particles in Wigner's view are in agreement with Solèr's picture in complex Hilbert spaces. Indeed, these systems are described as irreducible unitary representations of Poincaré group and, supposing that the von Neumann algebra of observables is that generated by the representation, Schur's lemma demonstrates that the algebra of observables is the entire $\mathfrak{B}(H)$. Therefore the lattice of elementary propositions is the entire $\mathcal{L}(\mathrm{H})$ in agreement with the thesis of Solèr's theorem. What happens when changing the set of scalars of the Hilbert space, passing 
from $\mathbb{C}$ to $\mathbb{R}$ or $\mathbb{H}$ is not obvious.

\subsection{Theoretical notions in common with the three types of Hilbert spaces formulations}

The following theoretical notions used to axiomatise quantum theories are defined in a separable Hilbert space $\mathrm{H}$, with scalar product $(\cdot \mid \cdot)$, over $\mathbb{R}, \mathbb{C}$ or $\mathbb{H}$ respectively and referring to the $\sigma$-complete orthocomplemented lattice $\mathcal{L}(\mathrm{H})$ of orthogonal projectors in $\mathrm{H}$. However these notions are defined also replacing $\mathcal{L}(\mathrm{H})$ for a smaller lattice $\mathcal{L}_{1}(\mathrm{H}) \subset \mathcal{L}(\mathrm{H})$, provided it is still orthocomplemented and $\sigma$-complete (and therefore also orthomodular and separable). For future convenience, we shall list these notions below in this generalized case. This list is more or less identical to that appearing in the introduction of [23].

(1) Elementary observables are represented by the orthogonal projectors in $\mathcal{L}_{1}(H)$. Two such projectors are said to be compatible if they commute as operators. Indeed the abstract commutativity notion of elementary observables turns out to be equivalent to the standard commutativity of associated orthogonal projectors [4, 22,

(2) Observables are the Projector-Valued Measures (PVMs) over the real Borel sets (see [23] for the real and complex case and [13] for the quaternionic case), taking values in $\mathcal{L}_{1}(\mathrm{H})$

$$
\mathcal{B}(\mathbb{R}) \ni E \mapsto P^{(A)}(E) \in \mathcal{L}_{1}(\mathrm{H}) .
$$

Equivalently, [40] an observable is a selfadjoint operator $A: D(A) \rightarrow \mathrm{H}$ with $D(A) \subset \mathrm{H}$ a dense subspace such that the associated projector-valued measure is made by elements of $\mathcal{L}_{1}(\mathrm{H})$. The link with the previous notion is the statement of the spectral theorem for selfadjoint operators $A=\int_{\sigma(A)} \lambda d P^{(A)}(\lambda)$ (23] for the real and complex case, for the quaternionic case see [13]).

Obviously the meaning of each elementary proposition $P^{(A)}(E)$ is the outcome of the measurement of $A$ belongs to the real Borel set $E$.

Evidently, $\mathcal{L}_{1}(\mathrm{H})=\mathcal{L}(\mathrm{H})$ if and only if every selfadjoint operator in $\mathrm{H}$ represents an observable. A selfadjoint operator, in particular an observables, $A$ is said to be compatible with another selfadjoint operator, in particular an observables, $B$ when the respective PVMs are made of pairwise commuting projectors.

(3) Quantum states are defined as $\sigma$-additive probability measures over $\mathcal{L}_{1}(\mathrm{H})$, that is maps $\mu: \mathcal{L}_{1}(\mathrm{H}) \rightarrow[0,1]$ such that $\mu(I)=1$ and

$$
\mu\left(s-\sum_{k} P_{k}\right)=\sum_{k} \mu\left(P_{k}\right) \quad \text { if }\left\{P_{k}\right\}_{k \in \mathbb{N}} \subset \mathcal{L}_{1}(\mathrm{H}) \text { with } P_{k} P_{h}=0 \text { for } h \neq k,
$$

$s-\sum_{k}$ denoting the series in the strong operator topology. $\mu(P)$ has the meaning of the probability that the outcome of $P$ is 1 if the proposition is tested when the state is $\mu$.

If $\mathcal{L}_{1}(\mathrm{H})=\mathcal{L}(\mathrm{H})$ for $\mathrm{H}$ separable with $+\infty \geq \operatorname{dim}(\mathrm{H}) \neq 2$ (always assumed henceforth), these measures are in one-to-one correspondence with all of the selfadjoint positive, unittrace, trace-class operators $T_{\mu}: \mathrm{H} \rightarrow \mathrm{H}$ according to

$$
\mu(P)=\operatorname{tr}\left(P T_{\mu} P\right) \quad \forall P \in \mathcal{L}(H) .
$$


$T \in \mathfrak{B}(\mathrm{H})$ is trace-class [24] if

$$
\sum_{u \in N}\langle u|| T \mid u\rangle<+\infty \quad \text { for some Hibert basis } N \subset \mathrm{H}
$$

where $\mathrm{H}$ is a real, complex or quaternionic Hilbert space. The set of trace-class operators turns out to be a closed two-sided *-ideal of $\mathfrak{B}(\mathrm{H})$ (the unital real $C^{*}$-algebra of bounded operators $A: \mathrm{H} \rightarrow \mathrm{H}$ ) in the three considered cases [24].

This correspondence between $\mu$ and $T_{\mu}$ exists for the three cases as demonstrated by the celebrated Gleason's theorem valid for $\mathbb{R}$ and $\mathbb{C}[10$. The quaternionic case is more complicated and the extension proposed by Varadarajan in [40] was partially incorrect. A correct statement has been recently obtained by the authors of this paper [24]. The problem is that the notion of trace in quaternionic Hilbert space is necessarily basis dependent unless the argument of the trace is selfadjoint. Above $P T_{\mu} P$ is explicitely selfadjoint and the cyclic property of the trace together with $P P=P$ proves that $\operatorname{tr}\left(P T_{\mu} P\right)=\operatorname{tr}\left(P T_{\mu}\right)$ in the complex and real cases, finding the standard statement of Gleason theorem in those cases. Cyclicity of the trace does not hold in the quanternionic case [24]. An alternative, equivalent, and much more effective approach [24] is to state Gleason's identity in the three cases as

$$
\mu(P)=\operatorname{Re}\left(\operatorname{tr}\left(P T_{\mu}\right)\right) \quad \forall P \in \mathcal{L}(H),
$$

where, for a quaternionic Hilbert space, $\operatorname{tr}\left(P T_{\mu}\right)$ is computed on a Hilbert basis fixed arbitrarily. In fact, it turns out that the real part of the trace is basis independent in quaternionic Hilbert space (and also in the remaining two cases). Finally (1) is equivalent to (2) because $\operatorname{Re}\left(\operatorname{tr}\left(P T_{\mu}\right)\right)=\operatorname{tr}\left(P T_{\mu} P\right)$ by elementary properties of the trace operation in the three considered cases (see [24] for details).

Gleason's result is valid (but the correspondence of measures and positive unit-trace thrace-class operators ceases to be one-to-one) for separable complex Hilbert spaces when $\mathcal{L}_{1}(\mathrm{H}) \subsetneq \mathcal{L}(\mathrm{H})$ and $\mathcal{L}_{1}(\mathrm{H})$ is the projector lattice of a von Neumann algebra whose canonical decomposition into definite-type von Neumann algebras does not contain type- $I_{2}$ algebras [6].

(4) Pure states are extremal elements of the convex body of the afore-mentioned probability measures. If $\mathcal{L}_{1}(\mathrm{H})=\mathcal{L}(\mathrm{H})$ pure states are one-to-one with unit vectors of $\mathrm{H}$ up to (generalized) phases $\eta$, i.e., numbers of $\mathbb{R}, \mathbb{C}, \mathbb{H}$ respectively, with $|\eta|=1$. In this case, the notion of probability transition $|\langle\psi \mid \phi\rangle|^{2}$ of a pair of pure states defined by unit vectors $\psi, \phi$ can be introduced. $|\langle\psi \mid \phi\rangle|^{2}=\mu_{\psi}\left(P_{\phi}\right)$ is the probability that $P_{\phi}$ is true when the state is $\mu_{\psi}$, where $P_{\phi}=\langle\phi \mid \cdot\rangle \phi$ and $\mu_{\psi}:=\langle\psi \mid \cdot \psi\rangle$.

(5) Lüders-von Neumann's post measurement axiom can be formulated in the standard way in the three cases: If the outcome of the ideal measurement of $P \in \mathcal{L}_{1}(\mathrm{H})$ in the state $\mu$ is 1 , the post measurement state is

$$
\mu_{P}(\cdot):=\frac{\mu(P \cdot P)}{\mu(P)}
$$


If $\mathcal{L}_{1}(\mathrm{H})=\mathcal{L}(\mathrm{H})$, we may define states in terms of trace class operators and, with obvious notation, $T_{P}=\frac{1}{\operatorname{tr}(P T P)} P T P$. In terms of probability measures over $\mathcal{L}(\mathrm{H})$, this is equivalent to say that the post measurement measure $\mu_{P}$, when the state before the measurement of $P$ is $\mu$, is the unique probability-measure over $\mathcal{L}(\mathrm{H})$ satisfying the natural requirement of conditional probability $\mu_{P}(Q)=\frac{\mu(Q)}{\mu(P)}$, for every $Q \in \mathcal{L}(\mathrm{H})$ with $Q \leq P$.

(6) Symmetries are naturally defined as automorphisms $h: \mathcal{L}_{1}(\mathrm{H}) \rightarrow \mathcal{L}_{1}(\mathrm{H})$ of the lattice of elementary propositions. A subclass of symmetries $h_{U}$ are those induced by unitary (or also anti unitary in the complex case) operators $U \in \mathfrak{B}(\mathrm{H})$ by means of $h_{U}(P):=U P U^{-1}$ for every $P \in \mathcal{L}_{i}(\mathrm{H})$. Alternatively, another definition of symmetry is as automorphism of the Jordan algebra of observables constructed out of $\mathcal{L}_{1}(\mathrm{H})$. If $\mathcal{L}_{1}(\mathrm{H})=\mathcal{L}(\mathrm{H})$, following Wigner, symmetries can be defined as bijective probabilitytransition preserving transformations of pure states to pure states.

With the maximality hypothesis on the lattice, the three notions of symmetry coincide. In this case, all symmetries turn out to be described by unitary (or anti unitary in the complex case) operators, up to constant phases of $\mathbb{R}, \mathbb{C}, \mathbb{H}$, respectively due to well known theorems by Kadison, Wigner and Varadarajan [36, 40].

(7) Continuous symmetries are one-parameter groups of lattice automorphisms $\mathbb{R} \ni s \mapsto h_{s}$, such that $\mathbb{R} \ni s \mapsto \mu\left(h_{s}(P)\right)$ is continuous for every $P \in \mathcal{L}_{1}(\mathrm{H})$ and every quantum state $\mu$ ( $\mathbb{R}$ may be replaced for a topological group but we stick here to the simplest case). The time evolution of the system $\mathbb{R} \ni s \mapsto \tau_{s}$ is a preferred continuous symmetry parametrized over $\mathbb{R}$.

(8) A dynamical symmetry is a continuous symmetry $h$ which commutes with the time evolution, $h_{s} \circ \tau_{t}=\tau_{t} \circ h_{s}$ for $s, t \in \mathbb{R}$.

If $\mathcal{L}_{1}(\mathrm{H})=\mathcal{L}(\mathrm{H})$, every continuous symmetry $\mathbb{R} \ni s \mapsto h_{s}$ is represented by a strongly continuous one-parameter group of unitary operators $\mathbb{R} \ni s \mapsto U_{s}$ such that $h_{s}(P)=$ $U_{s} P U_{s}^{-1}$ for all $P \in \mathcal{L}(\mathrm{H})$ [40]. Versions of Stone theorem hold in the three considered cases $\mathbb{R}, \mathbb{C}$ and $\mathbb{H}$ (the validity in the quaternionic case easily arises form the theory developed in [13. and we will present a proof in Sect. 2.3), proving that $U_{s}=e^{s A}$ for some anti-selfadjoint operator $A$, uniquely determined by $U$. In the complex case, if $\mathbb{R} \ni s \mapsto e^{s A}$ is also a dynamical symmetry, the selfadjoint operator $-i A$, which is an observable the lattice being maximal, is invariant under the natural adjoint action of time evolution $\tau$ unitarily represented by $\mathbb{R} \ni t \mapsto V_{t}$, and thus $-i A$ is a constant of motion, $V_{t}^{-1}(-i A) V_{t}=-i A$ for every $t \in \mathbb{R}$. This is the celebrated quantum version of Noether theorem. In the real Hilbert space case, no such simple result exists, since we have no general way to construct a selfadjoint operator out of an anti selfadjoint operator $A$ in absence of $i$. There is no unitary operator $J$ corresponding to the imaginary unit $i I$ which commutes with the anti selfadjoint generator $A$ of every possible continuous symmetry (the time evolution in particular), thus producing an associated observable $J A$ which is a constant of motion. Such an operator however may exist for one or groups of observables. In the quaternionic case, contrarily, there are many, pairwise non-commuting, imaginary unities as recently established [13]. An interesting physical discussion on these partially open issues for the quaternionic formulation appears in [1]. 
(9) [Real and complex formulations only] Composite systems in real and complex Hilbert space formulations are simply described by taking the (Hilbert) tensor product of the Hilbert spaces of subsystems and constructing the corresponding tensor product structures (e.g., see [22]). Yet a fundamental obstruction arises with the quaternionic formulation, where a standard notion of tensor product is forbidden due to noncommutativity of quaternions. As a matter of fact, basic properties of the notion of the tensor product lead to the indentities $((u q) \otimes(v p))=(u \otimes(v p)) q=(u \otimes v) p q$ and $((u q) \otimes(v p))=((u q) \otimes v) p=(u \otimes v) q p$ producing the contradiction $p q=p q$. This is a long standing problem with some inconclusive attempt of solutions.

\subsection{Main results of this work}

As soon as one assumes the apparently quite natural hypotheses on the structure of the lattice of elementary propositions of a quantum system as stated in Solér's theorem, the above three Hilbert-space formulations are the only possibilities. Actually, as already remarked, Solér hypotheses appear to be quite rigid since they force the theory to be formulated in terms of type-I factors when looking at the von Neumann algebra of observables in the complex case. Though it is the standard picture in quantum mechanics (in a single superselection sector), it rules out quantum field theory and statistical mechanics of extend systems. In [23] we focused on a real-Hilbert space formulation without assuming all Solér hypotheses but supposing some restrictions concerning symmetries. As a matter of fact, we considered a weaker and more general notion of Wigner elementary relativistic systems and we proved that a natural relativistic-invariant complex structure with a very precise physical meaning exists in the real Hilbert space formulation, making complex the theory. This complex formulation also removes some redundancies present in the real formulation. In particular all complex-linear self-adjoint operators are observables, whereas many real-linear self-adjoint operators cannot be interpreted as observables in the real formulation and the correspondence between pure-states and rays is one-toone in the complex formulation but not in the real one. The passage from the real to the complex theory also permits one to recover the standard relation between continuous symmetries and conserved observables along the evolution of the system. Here we focus on elementary relativistic systems initially described in quaternionic Hilbert spaces.

The main goal and result of this work is the proof that, also in the case of a quaternionic formulation with Poincaré symmetry, the theory can be re-formulated into a standard complex Hilbert space picture, where all self-adjoint operators represent observables, the standard version of Noether relation between continuous symmetries and conserved observables is restored and the notion of composite system can be implemented by the standard tensor product. The complex structure is, as in [23], uniquely imposed by Poicaré symmetry and is Poincaré invariant.

We shall establish this result into a pair of distinct theorems. In Theorem 7.10 we prove the thesis referring to a notion of relativistic elementary system very close to Wigner's one: an irreducible strongly continuous representation of Poincaré group with non neg- 
ative squared mass, whose observables are determined by the representation itself. A second version of the result is proved in Theorem 8.9. Here a much more sophisticated notion of relativistic elementary system is adopted defined in terms of an irreducible von Neumann algebra of observables whose lattice of orthogonal projector admits a continuous (with respect to a physically meaningful topology) irreducible representation of Poincaré group and such that the algebra itself is determined by the representation in view of a generalised version of Wigner's theorem.

Differently from the real Hilbert space case, here we need an intermediate non-obvious technical result concerning the notion of von Neumann algebra in quanternionic Hilbert space. Indeed, in section 2.1, relying upon the spectral theory developed in [11] and [13], we state and prove the quaternionic version of the celebrated double commutant theorem. A new proof is necessary because the standard argument (e.g., see [15]) does not work due the non-commutativity of the algebra of quaternions. With the help of the von Neumann algebra machinery and extending some well-known results of the theory or representations of Lie groups we will achieve our final result after having introduced a (weaker) extension of Wigner's notion of elementary relativistic system 1 .

\section{Quaternionic operators and their natural structures}

\section{1 von Neumann algebras in quaternionic Hilbert spaces}

This section is devoted to extending the notion of von Neumann algebra to quaternionic Hilbert spaces. The overall idea is to extend classical von Neumann's result known as double commutant theorem to algebras of quaternionic right-linear operators and to define the notion of von Neumann algebra exploiting that result. The way to do it essentially is a reduction procedure from the quaternionic to the real and complex cases where the theory of von Neumann algebras is well established. This goal needs a few preliminary elementary results and constructions we shall employ also in other parts of this work.

As a first step we associate a quaternionic Hilbert space $\mathrm{H}$ with a corresponding real Hilbert space $\mathrm{H}_{\mathbb{R}}$ and a corresponding complex Hilbert space $\mathrm{H}_{\mathbb{C}_{j}}$ and we study the interplay of these structures and of the corresponding operator algebras. We remind the reader that, as previously stressed, only real linear combinations of quaternionic-linear operators can be performed so that for instance $\mathfrak{B}(\mathrm{H})$ is a real $C^{*}$-algebra and not a quaternionic one.

Proposition 2.1 Let $(\mathrm{H},\langle\cdot \mid \cdot\rangle)$ be a quaternionic Hilbert space and consider the associated real vector space on the set of vectors $\mathrm{H}_{\mathbb{R}}:=\mathrm{H}$, whose real linear combinations are the quaternionic ones with real coefficients, and define the real scalar product $(\cdot \mid \cdot):=\operatorname{Re}\langle\cdot \mid \cdot\rangle$ on $\mathrm{H}_{\mathbb{R}}$. The following facts are true.

(a) The norms of $\mathrm{H}$ and $\mathrm{H}_{\mathbb{R}}$ coincide and $\left(\mathrm{H}_{\mathbb{R}},(\cdot \mid \cdot)\right)$ is a real Hilbert space.

\footnotetext{
${ }^{1}$ The idea of the proof that is very similar to that exploited in the real Hilbert space case [23] appears in www.mi.infn.it/ vacchini/talks_bell17/Oppio.pdf after a talk of one of the authors given at the University of Milan (Department of Physics) on June 16th 2016.
} 
(b) Referring to the $\mathbb{R}$-linear operators $\mathcal{J}, \mathcal{K} \in \mathfrak{B}\left(\mathrm{H}_{\mathbb{R}}\right)$ defined by

$$
\mathcal{J} u:=u j, \quad \mathcal{K} u:=u k \quad \forall u \in \mathrm{H}_{\mathbb{R}}
$$

satisfying $\mathcal{J} \mathcal{J}=\mathcal{K K}=-I$ and $\mathcal{J K}=-\mathcal{K} \mathcal{J}$, we have

$$
\langle x \mid y\rangle=(x \mid y)-(x \mid \mathcal{J} y) j-(x \mid \mathcal{K} y) k-(x \mid \mathcal{J} \mathcal{K} y) j k \quad \forall x, y \in \mathrm{H}
$$

(c) Every quaternionic-linear operator is real-linear and in particular $\mathfrak{B}(\mathrm{H}) \subset \mathfrak{B}\left(\mathrm{H}_{\mathbb{R}}\right)$, where $\mathfrak{B}(\mathrm{H})$ is the unital real $C^{*}$-algebra of bounded operators on $\mathrm{H}$ and the inclusion of algebras preserves the norms of the operators.

(d) $A \in \mathfrak{B}\left(\mathrm{H}_{\mathbb{R}}\right)$ belongs to $\mathfrak{B}(\mathrm{H})$ if and only if it commutes with both $\mathcal{J}$ and $\mathcal{K}$.

(e) Let $A: D(A) \rightarrow \mathrm{H}$ be a quaternionic-linear operator with $D(A) \subset \mathrm{H}$ a (quaternionic) subspace and consider $A$ as a real-linear operator on $\mathrm{H}_{\mathbb{R}}$ with domain $D(A)$ viewed as real subspace of $\mathrm{H}_{\mathbb{R}}$. Then

(i) $\mathcal{J} A \subset A \mathcal{J}$ and $\mathcal{K} A \subset A \mathcal{K}$,

(ii) $D(A)$ is a dense real-linear subspace of $\mathrm{H}_{\mathbb{R}}$ if it is dense in $\mathrm{H}$,

(iii) the adjoint of $A$ with respect to $\mathrm{H}$ and with respect to $\mathrm{H}_{\mathbb{R}}$ coincide,

(iv) A is symmetric, antisymmetric, essentially selfadjoint, essentially antiselfadjont, selfadjoint, antiselfadjoint, isometric, unitary, idempotent in $\mathrm{H}_{\mathbb{R}}$ if it is respectively such in $\mathrm{H}$.

(v) $A$ is closable in $\mathrm{H}_{\mathbb{R}}$ if it is such in $\mathrm{H}$. In this case the closures defined in $\mathrm{H}$ and $\mathrm{H}_{\mathbb{R}}$, respectively coincide,

(vi) $A$ is positive on $\mathrm{H}_{\mathbb{R}}$ if it is such on $\mathrm{H}$.

(f) Let $A: D(A) \rightarrow \mathrm{H}_{\mathbb{R}}$ be a $\mathbb{R}$-linear operator with $D(A) \subset \mathrm{H}_{\mathbb{R}}$ a (real) subspace and suppose that $\mathcal{J} A \subset A \mathcal{J}$ and $\mathcal{K} A \subset A \mathcal{K}$. Then

(i) $D(A)$ is a quaternionic-linear subspace of $\mathrm{H}$ which is dense if it is dense in $\mathrm{H}_{\mathbb{R}}$ and $A$ is a quaternionic linear operator.

(ii) the adjoint of $A$ with respect to $\mathrm{H}_{\mathbb{R}}$ and with respect to $\mathrm{H}$ coincide,

(iii) A is symmetric, antisymmetric, essentially selfadjoint, essentially antiselfadjont, selfadjoint, antiselfadjoint, isometric, unitary, idempotent in $\mathrm{H}$ if it is respecively such in $\mathrm{H}_{\mathbb{R}}$,

(iv) $A$ is closable in $\mathrm{H}$ if it is such in $\mathrm{H}_{\mathbb{R}}$. In this case the closure defined on $\mathrm{H}$ and $\mathrm{H}_{\mathbb{R}}$, respectively coincide,

(v) $A$ is positive in $\mathrm{H}$ if it is positive and symmetric in $\mathrm{H}_{\mathbb{R}}$.

Proof. All statements immediately follow from the corresponding definitions. 
We are now in a position to focus on the properties of the commutant of sets of quaternionic linear operators in $\mathfrak{B}(\mathrm{H})$, viewed as a unital real $C^{*}$-algebra, with the final aim to prove the quaternionic version of the double commutant theorem.

As in the real and complex Hilbert space cases, if $\mathfrak{S} \subset \mathfrak{B}(\mathrm{H})$ with $\mathrm{H}$ a quaternionic Hilbert space, that the commutant $\mathfrak{S}^{\prime}$ of $\mathfrak{S}$ is

$$
\mathfrak{S}^{\prime}:=\{A \in \mathfrak{B}(\mathrm{H}) \mid A S=S A \quad \forall S \in \mathfrak{S}\} .
$$

If $\mathfrak{S} \subset \mathfrak{T} \subset \mathfrak{B}(\mathrm{H})$, it is easy to see that $\mathfrak{T}^{\prime} \subset \mathfrak{S}^{\prime}$ and $\mathfrak{S} \subset \mathfrak{S}^{\prime \prime}$. This immediately leads to $\mathfrak{S}^{\prime \prime \prime}=\mathfrak{S}^{\prime}$. Moreover, if $\mathfrak{S}$ is closed under the ${ }^{*}$-operation, then its commutant $\mathfrak{S}^{\prime}$ turns out to be a unital ${ }^{*}$-subalgebra of the (real) unital $C^{*}$-algebra $\mathfrak{B}(\mathrm{H})$. All that is identical to the known results in real and complex Hilbert spaces. In view of the definition of $H_{\mathbb{R}}$, however, $\mathfrak{S} \subset \mathfrak{B}(\mathrm{H})$ can also be interpreted as a subset of $\mathfrak{B}\left(\mathrm{H}_{\mathbb{R}}\right)$ and thus a corresponding notion of commutant $\mathfrak{S}^{\prime \mathbb{R}} \subset \mathfrak{B}\left(\mathrm{H}_{\mathbb{R}}\right)$,

$$
\mathfrak{S}^{\prime \mathbb{R}}:=\left\{A \in \mathfrak{B}\left(\mathrm{H}_{\mathbb{R}}\right) \mid A S=S A \quad \forall S \in \mathfrak{S}\right\}
$$

can be defined. Notice that $\mathcal{J}, \mathcal{K}, \mathcal{J K} \in \mathfrak{S}^{\prime \mathbb{R}}$ whatever $\mathfrak{S} \subset \mathfrak{B}(\mathrm{H})$ we choose, it being made of quaternionic-linear operators due to (e) in Prop 2.1. An elementary result relating the two notions of commutant is the following lemma.

Lemma 2.2 For every $\mathfrak{S} \subset \mathfrak{B}(\mathrm{H})$, it holds

$$
\mathfrak{S}^{\prime} \subset \mathfrak{S}^{\prime \mathbb{R}} \subset \mathfrak{S}^{\prime}+\mathcal{J} \mathfrak{S}^{\prime}+\mathcal{K} \mathfrak{S}^{\prime}+\mathcal{J} \mathcal{K} \mathfrak{S}^{\prime} \quad\left(\subset \mathfrak{B}\left(\mathrm{H}_{\mathbb{R}}\right)\right) .
$$

Proof. The first inclusion is trivial since all $\mathbb{H}$-linear operators commuting with the elements of $\mathfrak{S}$ are $\mathbb{R}$-linear. Let us pass to the second inclusion. For $A \in \mathfrak{S}^{\text {/ }}$ define the operator $B:=A-\mathcal{J} A \mathcal{J}-\mathcal{K} A \mathcal{K}-\mathcal{J} \mathcal{K} A \mathcal{J K}$. This clearly belongs to $\mathfrak{S}^{\prime \mathbb{R}}$. By direct inspection one immediately sees that $B$ also commutes with both $\mathcal{J}$ and $\mathcal{K}$ and thus it is quaternionic linear ((f) Prop 2.1) and belongs to $\mathfrak{S}^{\prime}$. Similarly, if defining $B_{\mathcal{J}}:=A-\mathcal{J} A \mathcal{J}+\mathcal{K} A \mathcal{K}+\mathcal{J} \mathcal{K} A \mathcal{J K}$, then $\mathcal{J} B_{\mathcal{J}}$ turns out to belong to $\mathfrak{S}^{\prime}$. We can repeat a similar argument twice eventually establishing that

$$
\begin{cases}B & :=A-\mathcal{J} A \mathcal{J}-\mathcal{K} A \mathcal{K}-\mathcal{J K} A \mathcal{J K} \in \mathfrak{S}^{\prime} \\ B_{\mathcal{J}} & :=A-\mathcal{J} A \mathcal{J}+\mathcal{K} A \mathcal{K}+\mathcal{J} \mathcal{K} A \mathcal{J} \mathcal{J} \in \mathcal{J} \mathfrak{S}^{\prime} \\ B_{\mathcal{K}} & :=A-\mathcal{K} A \mathcal{K}+\mathcal{J} A \mathcal{J}+\mathcal{J} \mathcal{K} A \mathcal{J} \mathcal{K} \in \mathfrak{K S}^{\prime} \\ B_{\mathcal{J K}} & :=A-\mathcal{J K} A \mathcal{J K}+\mathcal{J} A \mathcal{J}+\mathcal{K} A \mathcal{K} \in \mathcal{J} \mathcal{K} \mathfrak{S}^{\prime}\end{cases}
$$

This concludes the proof because $4 A=B+B_{\mathcal{J}}+B_{\mathcal{K}}+B_{\mathcal{J K}} \in \mathfrak{S}^{\prime}+\mathcal{J} \mathfrak{S}^{\prime}+\mathcal{K} \mathfrak{S}^{\prime}+\mathcal{J} \mathcal{K} \mathfrak{S}^{\prime}$.

We can now state and prove the quaternionic version of the double commutant theorem. The proof of this key tool is different to the known one of real and complex *-algebras [15] in view of the non-commutativity of quaternions. A crucial intermediate result in the proof of Theorem $5.31\left[15\right.$ to get $\mathfrak{R}^{\prime \prime} \subset \overline{\mathfrak{R}}^{s}$ uses the fact that $\mathbf{H}_{\psi}:=\overline{\{A \psi \mid A \in \mathfrak{R}\}}$ 
for $\psi \in \mathrm{H}$ is a closed complex subspace of $\mathrm{H}$. If $\mathrm{H}$ is quaternionic the corresponding property is false since $\mathrm{H}_{\psi}$ is only a real subspace instead of quaternionic in view of noncommutativity of quaternions. This is the reason why we followed an alternative and a bit elaborate route.

To do it, observe that a unital sub ${ }^{*}$-algebra $\mathfrak{R} \subset \mathfrak{B}(\mathrm{H})$ is also a unital sub ${ }^{*}$-algebra $\mathfrak{R} \subset \mathfrak{B}\left(\mathrm{H}_{\mathbb{R}}\right)$.

Theorem 2.3 (Double commutant theorem) Let $\mathrm{H}$ be a quaternionic Hilbert space and $\mathfrak{R} \subset \mathfrak{B}(\mathrm{H})$ a unital sub ${ }^{*}$-algebra, then

$$
\mathfrak{R}^{\prime \prime}=\overline{\mathfrak{R}}^{s}=\overline{\mathfrak{R}}^{w}=\mathfrak{R}^{\prime \mathbb{R}^{\prime} \mathbb{R}},
$$

where the strong and weak closure above can indifferently be taken in $\mathfrak{B}(\mathrm{H})$ or $\mathfrak{B}\left(\mathrm{H}_{\mathbb{R}}\right)$.

Proof. The idea of the proof is to reduce to the analogous result for algebras of operators in real Hilbert spaces (e.g., Thm 2.26 in [23]). As a first step, we prove that the weak and strong closures of a set $\mathfrak{S} \subset \mathfrak{B}(\mathrm{H}) \subset \mathfrak{B}\left(\mathrm{H}_{\mathbb{R}}\right)$ do not depend on the choice of either the real or the quaternionic Hilbert space structure. Let us prove it for the strong closures first. If an operator $A \in \mathfrak{B}\left(\mathrm{H}_{\mathbb{R}}\right)$ is a strong-limit of elements of $\mathfrak{S}$ then it commutes with $\mathcal{J}, \mathcal{K}$ because every element of the sequence does and so it belongs also to $\mathfrak{B}(\mathrm{H})$. To conclude, since the norms of $\mathrm{H}$ and $\mathrm{H}_{\mathbb{R}}$ coincide, we easily see that $A$ is also a strong limit of elements of $\mathfrak{S}$ within $\mathfrak{B}(\mathrm{H})$. The opposite inclusion is similar, just remember that $\mathfrak{B}(\mathrm{H}) \subset \mathfrak{B}\left(\mathrm{H}_{\mathbb{R}}\right)$. Let us pass to the weak closures. Take $\left\{A_{n}\right\}_{n \in \mathbb{N}} \subset \mathfrak{S}$ and first suppose that it weakly converges to some $A \in \mathfrak{B}(\mathrm{H})$. This means that $\left\langle x \mid A_{n} y\right\rangle \rightarrow\langle x \mid A y\rangle$ for every $x, y \in \mathrm{H}$ and thus $\left(x \mid A_{n} y\right)=\operatorname{Re}\left(\left\langle x \mid A_{n} y\right\rangle\right) \rightarrow \operatorname{Re}(\langle x \mid A y\rangle)=(x \mid A y)$. $\mathbb{R}$-linearity of $A$ finally implies that $A_{n}$ weakly converges to $A \in \mathfrak{B}\left(\mathrm{H}_{\mathbb{R}}\right)$, too. Suppose conversely that $A_{n}$ weakly converges to $A \in \mathfrak{B}\left(\mathrm{H}_{\mathbb{R}}\right)$, that is $\left(x \mid A_{n} y\right) \rightarrow(x \mid A y)$ for every $x, y \in \mathrm{H}_{\mathbb{R}}$. $A$ is quaternionic-linear as every $A_{n}$ is. Indeed,

$$
\begin{aligned}
(x \mid \mathcal{J} A y) & =\left(\mathcal{J}^{*} x \mid A y\right)=\lim _{n \rightarrow \infty}\left(\mathcal{J}^{*} x \mid A_{n} y\right)=\lim _{n \rightarrow \infty}\left(x \mid \mathcal{J} A_{n} y\right)= \\
& =\lim _{n \rightarrow \infty}\left(x \mid A_{n} \mathcal{J} y\right)=(x \mid A \mathcal{J} y)
\end{aligned}
$$

Arbitrainess of $x, y$ yields $\mathcal{J} A=A \mathcal{J}$. The same arguments works for $\mathcal{K}$ and thus $A$ is $\mathbb{H}$-linear in view of (f) Prop 2.1. Finally $A_{n}$ converges weakly to $A$ also on $\mathrm{H}$ because (3) gives

$$
\begin{aligned}
\left\langle x \mid A_{n} y\right\rangle & =\left(x \mid A_{n} y\right)-\left(x \mid \mathcal{J} A_{n} y\right) j-\left(x \mid \mathcal{K} A_{n} y\right) k-\left(x \mid \mathcal{J} \mathcal{K} A_{n} y\right) j k= \\
& =\left(x \mid A_{n} y\right)-\left(x \mid A_{n} \mathcal{J} y\right) j-\left(x \mid A_{n} \mathcal{K} y\right) k-\left(x \mid A_{n} \mathcal{J} \mathcal{K} y\right) j k \\
& \rightarrow(x \mid A y)-(x \mid A \mathcal{J} y) j-(x \mid A \mathcal{K} y) k-(x \mid A \mathcal{J} \mathcal{K} y) j k=\langle x \mid A y\rangle
\end{aligned}
$$

We have in particular established that the weak and strong closures of a unital *-algebra $\mathfrak{R} \subset \mathfrak{B}(\mathrm{H}) \subset \mathfrak{B}\left(\mathrm{H}_{\mathbb{R}}\right)$ do not depend on the real or quaternionic Hilbert space structure. The double commutant theorem for real unital *-algebras guarantees that

$$
\left(\mathfrak{R}^{\prime \mathbb{R}}\right)^{\prime / \mathbb{R}}=\overline{\mathfrak{R}}^{s}=\overline{\mathfrak{R}}^{w}
$$


where now the closures can indifferently be intepreted in $\mathfrak{B}(\mathrm{H})$ or in $\mathfrak{B}\left(\mathrm{H}_{\mathbb{R}}\right)$.

To conclude the proof, take a $\mathbb{H}$-linear operator $A \in\left(\mathfrak{R}^{\prime}\right)^{\prime}$. $A$ is also $\mathbb{R}$-linear and thus $A \in\left(\mathfrak{R}^{\prime}\right)^{\prime \mathbb{R}}$ (where $\mathfrak{R}^{\prime}$ is first defined within $\mathfrak{B}(\mathrm{H})$ and next is viewed as a subset of $\left.\mathfrak{B}\left(\mathrm{H}_{\mathbb{R}}\right)\right)$. So $A$ is a $\mathbb{R}$-linear operator which commutes with $\mathcal{J}, \mathcal{K}$ and the elements of $\mathfrak{R}^{\prime}$. The second inclusion in the statement of Lemma 2.2, implies that $A$ also commutes with the elements of $\mathfrak{R}^{\prime \mathbb{R}}$. Summing up, $A \in\left(\mathfrak{R}^{\prime \mathbb{R}}\right)^{\prime \mathbb{R}}$. This proves that $\left(\mathfrak{R}^{\prime}\right)^{\prime} \subset\left(\mathfrak{R}^{\prime \mathbb{R}}\right)^{\prime \mathbb{R}}=\overline{\mathfrak{R}}^{s}$, the identity arising from (44). Since $\mathfrak{R} \subset\left(\mathfrak{R}^{\prime}\right)^{\prime}$, we also have that $\mathfrak{R} \subset\left(\mathfrak{R}^{\prime}\right)^{\prime} \subset\left(\mathfrak{R}^{\prime \mathbb{R}}\right)^{\prime \mathbb{R}}=\overline{\mathfrak{R}}^{s}$. Taking the strong closure of every space and noticing that $\mathfrak{S}^{\prime}$ and $\mathfrak{S}^{\prime \mathbb{R}}$ are always strongly closed, we have $\overline{\mathfrak{R}}^{s} \subset\left(\mathfrak{R}^{\prime}\right)^{\prime} \subset\left(\mathfrak{R}^{\prime \mathbb{R}}\right)^{\prime \mathbb{R}}=\overline{\mathfrak{R}}^{s}$ so that $\overline{\mathfrak{R}}^{s}=\left(\mathfrak{R}^{\prime}\right)^{\prime}=\left(\mathfrak{R}^{\prime \mathbb{R}}\right)^{\prime \mathbb{R}}$ and (41) concludes the proof of $\mathfrak{R}^{\prime \prime}=\overline{\mathfrak{R}}^{s}=\overline{\mathfrak{R}}^{w}=\mathfrak{R}^{\prime \mathbb{R}^{\prime} \mathbb{R}}$.

Corollary 2.4 Let $\mathrm{H}$ be a quaternionic Hilbert space and $\mathfrak{R} \subset \mathfrak{B}(\mathrm{H})$ a unital sub *algebra. The following statements are equivalent:

(a) $\mathfrak{R}=\mathfrak{R}^{\prime \prime}$,

(b) $\mathfrak{R}$ is weakly closed,

(c) $\mathfrak{R}$ is strongly closed.

Proof. (a) implies (b) from Thm 2.3, (b) implies (c) trivially. If (c) holds true then $\mathfrak{R}=\overline{\mathfrak{R}}^{s}=\mathfrak{R}^{\prime \prime}$ from Thm 2.3 again.

This result suggests to extend the usual definition of von Neumann algebra to the quaternionic case.

Definition 2.5 If $\mathrm{H}$ is a quaternionic Hilbert space, a von Neumann algebra in $\mathfrak{B}(\mathrm{H})$ is a unital ${ }^{*}$-subalgebra of $\mathfrak{B}(\mathrm{H})$ satisfying the three equivalent statements of Corollary 2.4.

\section{Remark 2.6}

(a) In view of $T h m, 2.3$, if $H$ is a quaternionic Hilbert space and $\mathfrak{R} \subset \mathfrak{B}(\mathrm{H})$ a unital *-algebra, then $\mathfrak{R}$ is a von Neumann algebra on $\boldsymbol{H}$ if and only if it is a von Neumann algebra on $\mathrm{H}_{\mathbb{R}}$.

(b) Exactly as in the real Hilbert space case, in quaternionic Hilbert spaces, a von Neumann algebra is a unital sub $C^{*}$-algebra of $\mathfrak{B}(\mathrm{H})\left(\right.$ and $\left.\mathfrak{B}\left(\mathrm{H}_{\mathbb{R}}\right)\right)$ since strong closure implies uniform closure and $\left\|A^{*} A\right\|=\|A\|^{2}$ for $A \in \mathfrak{B}(\mathrm{H})$ is valid also in quaternionic Hilbert spaces [11].

If $\mathfrak{S} \subset \mathfrak{B}(\mathbf{H})$ is *-closed, $\mathfrak{S}^{\prime}$ is a unital *-algebra which is von Neumann because $\mathfrak{S}^{\prime}=\left(\mathfrak{S}^{\prime}\right)^{\prime \prime}$, in particular, $\mathfrak{S}^{\prime \prime}=\left(\mathfrak{S}^{\prime}\right)^{\prime}$ is also a von Neumann algebra. If $\mathfrak{T} \supset \mathfrak{S}$ is another von Neumann algebra, we have $\mathfrak{T}^{\prime} \subset \mathfrak{S}^{\prime}$ and so $\mathfrak{T}^{\prime \prime} \supset \mathfrak{S}^{\prime \prime}$. Therefore $\mathfrak{S}^{\prime \prime}$ is the smallest von Neumann algebra including $\mathfrak{S}$.

Definition 2.7 Let $\mathfrak{S} \subset \mathfrak{B}(\mathrm{H})$ be closed under the ${ }^{*}$-operation, then $\mathfrak{S}^{\prime \prime}$ is called the von Neumann generated by $\mathfrak{S}$. 


\subsection{The lattice of orthogonal projectors of quaternionic von Neumann algebras}

An important structure associated with a von Neumann algebra $\mathfrak{R}$, in a quaternionic Hilbert space $\mathrm{H}$ in particular, is the lattice of orthogonal projectors in $\mathfrak{R}$, denoted by $\mathcal{L}_{\mathfrak{R}}(\mathrm{H})$ (where $\mathcal{L}(\mathrm{H}):=\mathcal{L}_{\mathfrak{B}(\mathrm{H})}(\mathrm{H})$ ). As in the real case [23] and differently from the complex case it does not contain all the information about the algebra $\mathfrak{R}$. Indeed differently from the case of a complex Hilbert space, it may hold $\mathcal{L}_{\mathfrak{R}}(\mathrm{H})^{\prime \prime} \subsetneq \mathfrak{R}$, as the following elementary example shows.

Example 2.8 Let $\mathrm{H}$ be any quaternionic Hilbert space and $J \in \mathfrak{B}(\mathrm{H})$ such that $J^{*}=-J$ and $J J=-I$. Consider the unital sub ${ }^{*}$-algebra $\mathfrak{R}:=\{a I+b J \mid a, b \in \mathbb{R}\} \subset \mathfrak{B}(\mathrm{H})$. If we manage to prove that $\mathfrak{R}$ is weakly closed, Corollary 2.4 guarantees that it is a von Neumann algebra. So, suppose there exists some $A \in \mathfrak{B}(\mathrm{H})$ such that $a_{n} I+b_{n} J \rightarrow A$ weakly. It is clear that $a_{n} I-b_{n} J=\left(a_{n} I+b_{n} J\right)^{*}$ weakly converges to $A^{*}$. So that $a_{n} \rightarrow a$ and $b_{n} \rightarrow b \in \mathbb{R}$ for some $a, b \in \mathbb{R}$. This gives $A=a I+b J$ so that $\mathfrak{R}$ is weakly closed and therefore is a von Neumann algebra. On the other hand, $\mathcal{L}_{\mathfrak{R}}(\mathrm{H})=\{0, I\}$, since every self-adjoint element of $\mathfrak{R}$ is necessarily of the form $a I$ for some $a \in \mathbb{R}$. The smallest algebra containing it is obviously $\mathcal{L}_{\mathfrak{R}}(\mathrm{H})^{\prime \prime}=\{a I \mid a \in \mathbb{R}\} \subsetneq \mathfrak{R}$.

To investigate the elementary properties of $\mathcal{L}_{\mathfrak{R}}(\mathrm{H})$ we need some further preliminary technical results. Similarly to Prop 2.1, given a quaternionic Hilbert space $\mathbf{H}$, and using the real Hilbert space $\mathrm{H}_{\mathbb{R}}$, we can define an associated complex Hilbert space $\mathrm{H}_{\mathbb{C}}$ depending on the choice of a preferred imaginary unit. As usual $\mathbb{C}_{j}=\{a+j b \mid a, b \in \mathbb{R}\}$ is a quaternionic realization of the field of complex numbers relying on the choice of the imaginary unit $j \in \mathbb{H}$.

Proposition 2.9 Let $(\mathrm{H},\langle\cdot \mid \cdot\rangle)$ be a quaternionic Hilbert space, fix the imaginary unit $j \in \mathbb{H}$ and a corresponding $\mathbb{R}$-linear operator $\mathcal{J}$ in $\mathrm{H}_{\mathbb{R}}$ as in (b) of Prop 2.1 and consider the associated complex vector space on the set of vectors $\mathrm{H}_{\mathbb{C}_{j}}:=\mathrm{H}$, whose complex linear combinations are the quaternionic ones with coefficients in $\mathbb{C}_{j}$, and define the Hermitian scalar product

$$
(x \mid y)_{j}:=(x \mid y)-(x \mid \mathcal{J} y) j \quad \forall x, y \in \mathrm{H},
$$

where $x, y$ are viewed as elements of $\mathrm{H}_{\mathbb{R}}$ and the corresponding real scalar product takes place in the right-hand side. The following facts are true.

(a) $(x \mid y)=\operatorname{Re}(x \mid y)_{j}$ for every $x, y \in \mathrm{H}$ viewed as elements of $\mathrm{H}_{\mathbb{R}}$.

(b) $\langle x \mid y\rangle=(x \mid y)_{j}-k(x \mid K y)_{j}$ for every $x, y \in \mathrm{H}$

(c) The norms of $\mathrm{H}, \mathrm{H}_{\mathbb{R}}$ and $\mathrm{H}_{\mathbb{C}_{j}}$ coincide and $\left(\mathrm{H}_{\mathbb{C}_{j}},(\cdot \mid \cdot)_{j}\right)$ is a complex Hilbert space. $\left(\mathrm{H}_{\mathbb{C}_{j}}=\left(\mathrm{H}_{\mathbb{R}}\right)_{\mathcal{J}}\right.$ where the right-hand side is the internal complexification of $\mathrm{H}_{\mathbb{R}}$ referred to the complex structure $\mathcal{J}$ as in Sect.2.5 in [23]).

(d) Every quaternionic-linear operator is complex-linear and in particular $\mathfrak{B}(\mathrm{H}) \subset \mathfrak{B}\left(\mathrm{H}_{\mathbb{C}_{j}}\right)$ and the inclusion of algebras preserves the norms of the operators. 
(e) $A \in \mathfrak{B}\left(\mathrm{H}_{\mathbb{C}_{j}}\right)$ belongs to $\mathfrak{B}(\mathrm{H})$ if and only if it commutes with $\mathcal{K}$.

(f) Let $A: D(A) \rightarrow \mathrm{H}$ be a quaternionic-linear operator with $D(A) \subset \mathrm{H}$ a (quaternionic) subspace and consider $A$ as a complex-linear operator on $\mathrm{H}_{\mathbb{C}_{j}}$ with domain $D(A)$ viewed as complex subspace of $\mathrm{H}_{\mathbb{C}_{j}}$. Then

(i) $\mathcal{K} A \subset A \mathcal{K}$,

(ii) $D(A)$ is a dense real-linear subspace of $\mathrm{H}_{\mathbb{C}_{j}}$ if it is dense in $\mathrm{H}$,

(iii) the adjoint of $A$ with respect to $\mathrm{H}$ and with respect to $\mathrm{H}_{\mathbb{C}_{j}}$ coincide,

(iv) A is symmetric, antisymmetric, essentially selfadjoint, essentially antiselfadjont, selfadjoint, antiselfadjoint, isometric, unitary, idempotent in $\mathrm{H}_{\mathbb{C}_{j}}$ if it is respectively such in $\mathrm{H}$,

(v) $A$ is closable in $\mathbf{H}_{\mathbb{C}_{j}}$ if it is such in $\mathrm{H}$. In this case the closures defined in $\mathrm{H}$ and $\mathrm{H}_{\mathbb{C}_{j}}$, respectively coincide

(vi) $A$ is positive in $\mathrm{H}_{\mathbb{C}_{j}}$ if it is such in $\mathrm{H}$

(g) Let $A: D(A) \rightarrow \mathrm{H}_{\mathbb{C}_{j}}$ be a $\mathbb{C}$-linear operator with $D(A) \subset \mathrm{H}_{\mathbb{C}_{j}}$ a (complex) subspace and suppose that $\mathcal{K} A \subset A \mathcal{K}$. Then

(i) $D(A)$ is a quaternionic-linear subspace of $\mathrm{H}$ which is dense if it is dense in $\mathrm{H}_{\mathbb{C}_{j}}$ and $A$ is a quaternionic linear operator.

(ii) the adjoint of $A$ with respect to $\mathrm{H}_{\mathbb{C}_{j}}$ and with respect to $\mathrm{H}$ coincide,

(iii) A is symmetric, antisymmetric, essentially selfadjoint, essentially antiselfadjont, selfadjoint, antiselfadjoint, isometric, unitary, idempotent in $\mathrm{H}$ if it is respecively such in $\mathrm{H}_{\mathbb{C}_{j}}$,

(iv) $A$ is closable on $\mathrm{H}$ if it is such in $\mathrm{H}_{\mathbb{C}_{j}}$. In this case the closures defined in $\mathrm{H}$ and $\mathrm{H}_{\mathbb{C}_{j}}$, respectively coincide

(v) $A$ is positive in $\mathrm{H}$ if it is positive and symmetric on $\mathrm{H}_{\mathbb{C}_{j}}$

Proof. All statements immediately follows from the corresponding definitions.

Let $(\mathrm{K},(\cdot \cdot \cdot))$ be a real, complex or quaternionic Hilbert space. Consider a, generally unbounded, selfadjoint operator $A$. Thanks to the Spectral Theorem (see, e.g., [23] for the real case and [13] for the quaternionic cast2) it can be spectrally decomposed as

$$
A=\int_{\mathbb{R}} \lambda d P, \quad D(A)=\left\{\left.x \in \mathbf{H}\left|\int_{\mathbb{R}}\right| \lambda\right|^{2} d \mu_{x}^{P}<\infty\right\}
$$

where $P: \mathcal{B}(\mathbb{R}) \rightarrow \mathcal{L}(\mathrm{H})$ is the projection-valued measure (PVM) uniquely associated to $A$ and $\mu_{x}^{P}(E)=\left(x \mid P_{E} x\right)$ is the positive valued finite measure associated with $P$. The spectral theorem for self-adjoint operators can be restated as follows.

\footnotetext{
${ }^{2}$ Concerning self-adjoint operators, we use the notation $d P$ instead of $d \mathcal{P}$ preferred in [13] in the integral spectral formula.
} 
Lemma 2.10 Let $(\mathrm{K},(\cdot \mid \cdot))$ be a real, complex or quaternionic Hilbert space and consider a, generally unbounded, selfadjoint operator $A: D(A) \rightarrow \mathrm{K}$ with $D(A)$ dense in $\mathrm{K}$. There exists a unique $P V M P$ on $\mathbb{R}$ such that, if $\mu_{x}^{P}(E):=\left(x \mid P_{E} x\right)$, then $i d \in L^{2}\left(\mathbb{R}, \mu_{x}^{P}\right)$ and $(x \mid A x)=\int_{\mathbb{R}} \lambda d \mu_{x}^{P}$ for all $x \in D(A)$

Proof. The PVM $P$ associated to $A$ by the spectral theorem satisfies the thesis. Suppose $Q$ is another PVM satisfying the above requirements and take $x \in D(A)$. In particular this means that $x \in D\left(A^{\prime}\right)$ where $A^{\prime}$ is the selfadjoint operator $\int_{\mathbb{R}} \lambda d Q$ and $(x \mid A x)=$ $\left(x \mid A^{\prime} x\right)$, i.e. $\left(x \mid\left(A-A^{\prime}\right) x\right)=0$. Since $A-A^{\prime}$ is symmetric on the dense domain $D(A)$, a straightforward calculation shows that $A-A^{\prime}=0$ on $D(A)$, i.e. $A \subset A^{\prime}$. Since $A$ is selfadjoint it does not admit proper selfadjoint extensions (see [11, 13] for the quaternionic case and [23] for the real case), that is $A=A^{\prime}$. Finally, the spectral Theorem guarantees that $Q=P$.

Once the PVM associated with $A$ is available, if $f: \mathbb{R} \rightarrow \mathbb{R}$ is Borel measurable, the following operator can be defined

$$
f(A):=\int_{\mathbb{R}} f(\lambda) d P, \quad D(f(A)):=\left\{\left.x \in \mathrm{H}\left|\int_{\mathbb{R}}\right| \lambda\right|^{2} d \mu_{x}^{P}<\infty\right\}
$$

In particular it satisfies $(x \mid f(A) x)=\int_{\mathbb{R}} f(\lambda) d \mu_{x}^{P}$ for all $x \in D(f(A))$.

Another technical lemma is in order.

Lemma 2.11 Let $A$ be a, generally unbounded, self-adjoint operator on a quaternionic Hilbert space $\mathrm{H}$ with $P V M P^{(A)}$ and $U \in \mathfrak{B}(\mathrm{H})$. The following facts hold:

(a) $P^{(A)}$ equals the PVMs associated with $A$ on $\mathrm{H}_{\mathbb{R}}$ and $\mathrm{H}_{\mathbb{C}_{j}}$ via spectral theorem.

(b) $U A \subset A U$ if and only if $U P_{E}^{(A)}=P_{E}^{(A)} U$ for every $E \in \mathcal{B}(\mathbb{R})$.

(c) If $f: \mathbb{R} \rightarrow \mathbb{R}$ is Borel measurable, then the definitions of $f(A)$ referred to $\mathrm{H}, \mathrm{H}_{\mathbb{R}}$ or $\mathrm{H}_{\mathbb{C}_{j}}$ coincide.

Proof. Consider the unique real PVM $Q$ associated with $A$ on the real Hilbert space $\left(\mathrm{H}_{\mathbb{R}},(\cdot \mid \cdot)\right)$, while $P$ denotes the quaternionic analogue on $\mathrm{H}$. Take $u \in D(A)$. Since $A$ selfadjoint, it holds $\langle u \mid A u\rangle \in \mathbb{R}$, hence

$$
\int_{\mathbb{R}} \lambda d \mu_{u}^{Q}=(u \mid A u)=\langle u \mid A u\rangle=\int_{\mathbb{R}} \lambda d \mu_{u}^{P}
$$

It is easy to see that $P$ is still a PVM if understood on $\mathrm{H}_{\mathbb{R}}$. Moreover $\mu_{u}^{P}(E)=\left\langle u \mid P_{E} u\right\rangle=$ $\left(u \mid P_{E} u\right)$, again from selfadjointness of $P_{E}$. Lemma 2.10 for the real Hilbert space case proves that $P=Q$. We have established (a) for $\mathrm{H}_{\mathbb{R}}$. Next (b) follows immediately, since the statement is a well-known property in real Hilbert spaces [23]. Analogously, property (c) for $\mathrm{H}_{\mathbb{R}}$ follows easily from (a) using the fact that the integral operator in (5) can be computed as a strong limit (even uniform if $A$ is bounded) of bounded operators of the 
form $\sum_{l=1}^{n} c_{l} P\left(F_{l}\right)$ where $c_{l} \in \mathbb{R}$ and $F_{l} \in \mathcal{B}(\mathbb{R})$ depend only on the chosen function $f$ and not on the scalar field of the Hilbert space. Finally, the proofs for the case of ${H_{\mathbb{C}_{j}}}_{\text {is }}$ immediate by noticing that $\mathrm{H}_{\mathbb{C}_{j}}=\left(\mathrm{H}_{\mathbb{R}}\right)_{\mathcal{J}}$ and using the theory of [23].

We are eventually in a position to prove an elementary though relevant proposition concerning the interplay of $\mathfrak{R}$ and $\mathcal{L}_{\mathfrak{R}}(\mathrm{H})$. This result extends Thm 2.29 we established in [23] to quaternionic Hilbert spaces.

Proposition 2.12 Let $\mathfrak{R}$ a von Neumann algebra, define the set

$$
\mathcal{J}_{\mathfrak{R}}:=\left\{J \in \mathfrak{R} \mid J^{*}=-J,-J^{2} \in \mathcal{L}_{\mathfrak{R}}(\mathrm{H})\right\} .
$$

The following facts are valid.

(a) $A^{*}=A \in \Re$ iff the orthogonal projectors of its PVM belong to $\mathcal{L}_{\mathfrak{R}}(\mathrm{H})$.

(b) $\mathcal{L}_{\mathfrak{R}}(\mathrm{H})$ is a complete orthomodular bounded sublattice of $\mathcal{L}(\mathrm{H})$.

(c) $\mathcal{L}_{\mathfrak{R}}(\mathrm{H})^{\prime \prime}$ contains all the selfadjoint operators of $\mathfrak{R}$.

(d) $\mathcal{L}_{\mathfrak{R}}(\mathrm{H})^{\prime \prime}+\mathcal{J}_{\mathfrak{R}} \mathcal{L}_{\mathfrak{R}}(\mathrm{H})^{\prime \prime}=\mathfrak{R}$.

(e) $\mathcal{L}_{\mathfrak{R}}(\mathrm{H})^{\prime \prime} \subsetneq \mathfrak{R}$ iff there exists $J \in \mathcal{J}_{\mathfrak{R}} \backslash \mathcal{L}_{\mathfrak{R}}(\mathrm{H})^{\prime \prime}$.

Proof. (a) and (c). Suppose $A^{*}=A \in \mathfrak{R}$ and $B \in \mathfrak{R}^{\prime}$. In particular $B$ commutes with $A$ and Lemma 2.11 guarantees that $B$ commutes with the PVM $P^{(A)}$ of $A$. Arbitrariness of $B$ implies $P_{E}^{(A)} \in \mathfrak{R}^{\prime \prime}=\mathfrak{R}$ for every Borel set $E$. Suppose conversely that $A^{*}=A \in \mathfrak{B}(\mathrm{H})$ is such that $P_{E}^{(A)} \in \mathcal{L}_{\mathfrak{R}}(\mathrm{H})$ for every Borel set $E$, we want to prove that $A \in \mathfrak{R}$. If $B \in \mathcal{L}_{\mathfrak{R}}(\mathrm{H})^{\prime}$, in particular $B$ commutes with the PVM of $A$ and so, thanks again to Lemma 2.11, it commutes also with $A$ and $A \in \mathcal{L}_{\mathfrak{R}}(\mathrm{H})^{\prime \prime} \subset \Re$. This concludes the proof of (a). The used argument proves also (c). Indeed, if $A^{*}=A \in \mathfrak{R}$, then, thanks to the first implication of (a) its PVM belongs to $\mathcal{L}_{\mathfrak{R}}(\mathrm{H})$ and so the argument above applies.

(b) The properties of $\mathcal{L}_{\mathfrak{R}}(\mathrm{H})$ listed in (b) are inherited from the same properties of $\mathcal{L}(\mathrm{H})$. The proof can be obtained by mimicking the one developed in [32] for real and complex von Neumann algebras.

(d) First suppose $A \in \mathfrak{R}$ is anti selfadjoint. The polar decomposition theorem for bounded operators in quaternionic Hilbert spaces (see [11]) guarantees that $A=W|A|$ where $W$ is an antiselfadjoint partial isometry and $|A|:=\sqrt{A^{*} A}$. Moreover $W$ and $|A|$ commute with each other and with every operator commuting with $A$. This guarantees that $W,|A| \in$ $\mathfrak{R}^{\prime \prime}=\mathfrak{R}$. As $|A|$ is self-adjoint, $|A| \in \mathcal{L}_{\mathfrak{R}}(\mathrm{H})^{\prime \prime}$ for (c). Since $W$ is a partial isometry, $W^{2}$ is an orthogonal projector which clearly belongs to $\mathfrak{R}$, hence $W \in \mathcal{J}_{\mathfrak{R}}$. To conclude the proof of (d), observe that a generic operator $A \in \mathfrak{R}$ can always be decomposed as $\frac{1}{2}\left(A+A^{*}\right)+\frac{1}{2} W_{0}\left|A-A^{*}\right|$ where $\left(W_{0},\left|A-A^{*}\right|\right)$ is the polar decomposition of $A-A^{*}$. (c) and the previous discussion prove that $A+A^{*},\left|A-A^{*}\right| \in \mathcal{L}_{\mathfrak{R}}(\mathrm{H})^{\prime \prime}$ and $W_{0} \in \mathcal{J}_{\mathfrak{R}}$ concluding the proof of (d).

(e) If there exits $J \in \mathcal{J}_{\mathfrak{R}} \backslash \mathcal{L}_{\mathfrak{R}}(\mathrm{H})^{\prime \prime}$ then $\mathcal{L}_{\mathfrak{R}}(\mathrm{H})^{\prime \prime} \subsetneq \mathfrak{R}$ evidently. So, suppose $A \in \mathfrak{R} \backslash \mathcal{L}_{\mathfrak{R}}(\mathrm{H})^{\prime \prime}$, then $A-A^{*} \in \mathfrak{R} \backslash \mathcal{L}_{\mathfrak{R}}(\mathrm{H})^{\prime \prime}$, otherwise $A \in \mathcal{L}_{\mathfrak{R}}(\mathrm{H})^{\prime \prime}$ since $A+A^{*}$ is self-adjoint and thus belongs to $\mathcal{L}_{\mathfrak{R}}(\mathrm{H})^{\prime \prime}$ thanks to (c). Referring to the polar decomposition $A-A^{*}=$ $W_{0}\left|A-A^{*}\right|$, the above discussion guarantees that $W_{0} \in \mathcal{J}_{\mathfrak{R}}$. Since $A-A^{*} \notin \mathcal{L}_{\mathfrak{R}}(\mathrm{H})^{\prime \prime}$ and $\left|A-A^{*}\right| \in \mathcal{L}_{\mathfrak{R}}(\mathrm{H})^{\prime \prime}, W_{0}$ cannot belong to $\mathcal{L}_{\mathfrak{R}}(\mathrm{H})^{\prime \prime}$. 


\subsection{Stone theorem}

We need to extend to quaternionic Hilbert spaces the crucial technical result known as Stone Theorem. Consider an antiselfadjoint operator $A$ on $\mathrm{H}$. As $A$ is closed and normal [13], thanks to the spectral theorem for closed normal (generally unbounded) operators in quanternionic Hilbert spaces Theorem 6.6 in [13], fixed an imaginary unit $i$, there exists a unique PVM $P$ on $\mathbb{C}_{i}^{+}$and a (non-unique) left-multiplication $\mathcal{L}$ commuting with $P$, encapsulated in $\mathcal{P}:=(\mathcal{P}, \mathcal{L})$, such that

$$
A=\int_{\mathbb{C}_{i}^{+}} i d d \mathcal{P}(z), \quad D(A):=\left\{\left.x \in \mathrm{H}\left|\int_{\mathbb{C}_{i}}\right| z\right|^{2} d \mu_{x}^{(A)}<\infty\right\} .
$$

Notice that $\operatorname{supp} P \subset i \mathbb{R}^{+} \cup\{0\}$ since $A=-A^{*}$ (from (b) Thm 4.1 in [13] and (c) Thm 4.8 in [1]]). If $f: \mathbb{C}_{i}^{+} \rightarrow \mathbb{C}_{i}$ is Borel measurable we can define the operator

$$
f(A):=\int_{\mathbb{C}_{i}^{+}} f(z) d \mathcal{P}(z), \quad D(f(A)):=\left\{\left.x \in \mathrm{H}\left|\int_{\mathbb{C}_{i}^{+}}\right| f(z)\right|^{2} d \mu_{x}^{(A)}<\infty\right\}
$$

In particular, consider the operator

$$
e^{t A}:=\int_{\mathbb{C}_{i}^{+}} e^{t z} d \mathcal{P}(z) .
$$

The map $\mathbb{R} \ni t \mapsto e^{t A}$ is a one-parameter group of unitary operators as straightforward consequence of Thm 3.13 in [13]. Following essentially the same proof as in the complex Hilbert space case, one easily establishes that that map is strongly continuous and

$$
D(A)=\left\{x \in \mathrm{H}\left|\exists \frac{d}{d t}\right|_{0} U_{t} x \in \mathrm{H}\right\}, \quad A x=\left.\frac{d}{d t}\right|_{t=0} U_{t} x
$$

Exactly as in the complex and real cases, these one-parameter groups are the only strongly-continuous one-parameter unitary groups on $\mathrm{H}$. It is the content of the wellknown Stone's theorem now extended to the quaternionic Hilbert space case.

Theorem 2.13 (Stone's Theorem) Let $\mathrm{H}$ be a quaternionic Hilbert space and $\mathbb{R} \ni t \mapsto$ $U_{t} \in \mathfrak{B}(\mathrm{H})$ be a strongly-continuous one-parameter unitary group, then there exists a unique anti-selfadjoint operator $A$ called the (anti-selfadjoint) generator of $U$ such that

$$
U_{t}=e^{t A} \quad \text { for all } t \in \mathbb{R} .
$$

A turns out to be defined as in (10) where $D(A)$ is dense and invariant under the action of $U$.

Finally $A$ coincides with the unique anti-selfadjoint operator in $\mathrm{H}_{\mathbb{R}}\left(\mathrm{H}_{\mathbb{C}_{j}}\right)$ that generates $U$ when understood as strongly-continuous unitary group of operators in $\mathrm{H}_{\mathbb{R}}$ (resp. $\mathrm{H}_{\mathbb{C}_{j}}$ ).

Proof. See Appendix A. 


\subsection{Polar decomposition of unbounded operators in quaternionic Hilbert spaces}

Another technical tool is the so-called polar decomposition of closed generally unbounded operators. We state the version in quaternionic Hilbert spaces together with some direct application.

Theorem 2.14 (Polar decomposition theorem) Let $\mathrm{H}$ be a quaternionic Hilbert space and $A: D(A) \rightarrow \mathrm{H}$ a $\mathbb{H}$-linear closed operator with $D(A)$ dense $\mathrm{H}$. The following facts hold.

(a) $A^{*} A$ is densely defined, positive and selfadjoint.

(b) There exists a unique pair of operators $U, P$ in $\mathrm{H}$ such that,

(i) $A=U P$ where in particular $D(P)=D(A)$

(ii) $P$ is selfadjoint and $P \geq 0$

(iii) $U \in \mathfrak{B}(\mathrm{H})$ is isometric on $\operatorname{Ran}(P)$ (and thus on $\overline{\operatorname{Ran}(P)}$ by continuity),

(iv) $\operatorname{Ker}(U) \supset \operatorname{Ker}(P)$.

The right-hand side of (i) is called the polar decomposition of $A$. It turns out that, in particular,

(1) $P=|A|:=\sqrt{A^{*} A}$ (defined by means of (6) with $\sqrt{z}:=0$ if $z \notin[0,+\infty)$.)

(2) $\operatorname{Ker}(U)=\operatorname{Ker}(A)=\operatorname{Ker}(P)$,

(3) $\operatorname{Ran}(U)=\overline{\operatorname{Ran}(U)}$

(c) The polar decompositions of $A$ carried out on $\mathrm{H}, \mathrm{H}_{\mathbb{R}}, \mathrm{H}_{\mathbb{C}_{j}}$ are made of the same pair of operators $U, P$ arising in (i)(a) above.

Proof. See Appendix A.

A pair of related results, in part involving one-parameter groups of unitaries, are stated below.

Proposition 2.15 Let $\mathrm{H}$ be quaternionic Hilbert space. Consider an, either selfadjoint or anti selfadjoint, operator $A: D(A) \rightarrow \mathrm{H}$ with polar decomposition $A=U P$. The following facts hold.

(a) If $A^{*}=-A$, then $B \in \mathfrak{B}(\mathrm{H})$ satisfies $B e^{t A}=e^{t A} B$ if and only if $B A \subset A B$

(b) If $B \in \mathfrak{B}(\mathrm{H})$ satisfies $B A \subset A B$, then $B U=U B$ and $B P \subset U P$.

(c) The commutation relations are true

$$
U A \subset A U \text { and } U^{*} A \subset A U^{*} .
$$

Moreover, for every measurable function $f:[0,+\infty) \rightarrow \mathbb{R}$ :

$$
U f(P) \subset f(P) U \quad \text { and } \quad U^{*} f(P) \subset f(P) U^{*} .
$$


(d) $U$ is respectively selfadjoint or anti selfadjoint.

(e) If $A$ is injective (equivalently if either $P$ or $U$ is injective), then $U$ and $U^{*}$ are unitary. In this case all the inclusions in (c) are identies.

Proof. The thesis easily follows by working in $\mathrm{H}_{\mathbb{R}}$ and exploiting the analogous result for real Hilbert spaces, Thm 2.19 in [23]. Just notice that the definitions of $e^{t A}$ on $\mathrm{H}$ and $\mathbf{H}_{\mathbb{R}}$ coincide thanks to Theorem 2.13,

Proposition 2.16 Let $\mathrm{H}$ be a quaternionic Hilbert space and $A$ and $B$ anti-selfadjoint operators in $\mathrm{H}$ with polar decompositions $A=U|A|$ and $B=V|B|$. If the stronglycontinuous one-parameter groups generated by $A$ and $B$ commute, i.e.,

$$
e^{t A} e^{s B}=e^{s B} e^{t A} \quad \text { for every } s, t \in \mathbb{R}
$$

then the following facts hold

(a) $U B \subset B U$ and $U^{*} B \subset B U^{*}$;

(b) $U f(|B|) \subset f(|B|) U$ and $U^{*} f(|B|) \subset f(|B|) U^{*}$ for $f:[0,+\infty) \rightarrow \mathbb{R}$ every measurable function

(c) (iii) $U V=V U$ and $U^{*} V=V U^{*}$.

If any of $A,|A|, U$ is injective, then the inclusions in (i) and (ii) can be replaced by identities.

Proof. Again the thesis easily arises by working in $\mathrm{H}_{\mathbb{R}}$ and using the analogous result for real Hilbert spaces, Thm 2.20 in 23. Again notice that the definitions of $e^{t A}$ on $\mathrm{H}$ and $\mathrm{H}_{\mathbb{R}}$ coincide thanks to Theorem 2.13,

\section{Restriction to subspaces induced by complex structures}

To go on, we need to introduce some fundamental technical tools of quaternionic Hilbert spaces theory established in [11]. A preliminary definition is necessary.

Definition 3.1 If $\mathrm{H}$ is a quaternionic Hilbert space, an operator $S \in \mathfrak{B}(\mathrm{H})$ such that $S^{2}=-I$ and $S^{*}=-S$ is called complex structure on $\mathrm{H}$.

Remark 3.2 Notice that the previously introduced operators $\mathcal{J}$ and $\mathcal{K}$ are not complex structures because they are only $\mathbb{R}$-linear while complex structures $S$ are required to be $\mathbb{H}$-linear. 


\subsection{Restriction to a complex Hilbert subspace induced by a complex structure}

If $\mathrm{H}$ is a quaternionic Hilbert space with scalar product $\langle\cdot \mid \cdot\rangle, j, k$ any couple of anticommuting imaginary units and $J \in \mathfrak{B}(\mathrm{H})$ a complex structure, define the subsets (which should be indicated by $\mathrm{H}_{+}^{J j}$ and $\mathrm{H}_{-}^{J j}$ according to notation of [11])

$$
\mathrm{H}_{J}:=\{u \in \mathrm{H} \mid J u=u j\}, \quad \mathrm{H}_{J}^{(-)}:=\{u \in \mathrm{H} \mid J u=-u j\}
$$

$\mathrm{H}_{J}$ and $\mathrm{H}_{J}^{(-)}$are evidently closed by (right) scalar multiplication with quaternions $a+b j \in$ $\mathbb{C}_{j}$ and thus are complex vector spaces. It is easily proved that the restriction of $\langle\cdot \mid \cdot\rangle$ to $\mathrm{H}_{J}$, resp., $\mathrm{H}_{J}^{(-)}$is a Hermitian complex scalar product. Since these sets are evidently closed because $J$ is unitary, $\mathrm{H}_{J}$ and $\mathrm{H}_{J}^{(-)}$equipped with the relevant restrictions of $\langle\cdot \mid \cdot\rangle$ are complex Hilbert spaces. Since $j k=-k j$ the following identity holds

$$
\mathrm{H}_{J}^{(-)}=\mathrm{H}_{J} k:=\left\{v k \in \mathrm{H} \mid v \in \mathrm{H}_{J}\right\} .
$$

Due to (12), we will refer to $\mathrm{H}_{J}$ only in the rest of this paper.

Notice that the elements of $\mathrm{H}_{J}$ can be interpreted as complex vectors of $\mathrm{H}$, as they commute with every element of $\mathbb{C}_{j}$.

The following proposition collects some results that can be found in Prop.3.8, Lemmata 3.9-3.10 and the proof of (a) Prop. 3.11 of [11]. In particular

Proposition 3.3 Let $\mathrm{H}$ be a quaternionic Hilbert space and $J \in \mathfrak{B}(\mathrm{H})$ a complex structure. The complex vector space $\mathrm{H}_{J}$ in Def.(11) equipped with the complex-linear structure $\mathbb{C}_{j}$ and the restriction $\langle\cdot \mid \cdot\rangle_{J}$ of the scalar product of $\mathrm{H}$ to $\mathrm{H}_{J}$ satisfies the following properties.

(a) $\mathrm{H}_{J}$ is a $\mathbb{C}_{j}$-complex Hilbert space, non-trivial unless $\mathrm{H}=\{0\}$.

(b) The direct (generally not orthogonal) decomposition is valid $\mathrm{H}=\mathrm{H}_{J} \oplus \mathrm{H}_{J} k$ such that

$$
\|x\|^{2}=\left\|x_{1}\right\|^{2}+\left\|x_{2}\right\|^{2} \quad \text { if } \mathrm{H} \ni x=x_{1}+x_{2} k \text { with } x_{1}, x_{2} \in \mathrm{H}_{J} .
$$

(c) The map $\mathrm{H}_{J} \ni u \mapsto u k \in \mathrm{H}_{J} k$ is $\mathbb{C}_{j}$-antilinear, isometric and bijective.

(d) If $N \subset \mathrm{H}_{J}$ is a Hilbert basis of $\mathrm{H}_{J}$, then $N$ is also a Hilbert basis of the whole $\mathrm{H}$.

The complex space $\mathrm{H}_{J}$ has an important interplay with operators as established in Prop.3.11 in [11] we summarize below into a form adapted to our work. The statements (c)-(iii), (c)-(ix) and (d) do not appear in [11, however their proofs are trivial. In particular the proof of (c)-(iii) is analogous to the one of (c)-(iv).

Proposition 3.4 With the same hypotheses as for Prop 3.3 the following facts hold.

(a) If $A: D(A) \rightarrow \mathrm{H}$, with $D(A) \subset \mathrm{H}$, is $\mathbb{H}$-linear and $J A \subset A J$, then $A_{J}:=\left.A\right|_{\mathrm{H}_{J} \cap D(A)}$ is a well-defined $\mathbb{C}_{j}$-linear operator on $\mathrm{H}_{J}$.

(b) If $X: D(X) \rightarrow \mathrm{H}_{J}$, with $D(X) \subset \mathrm{H}_{J}$, is $\mathbb{C}_{j}$-linear, there exists a unique $\mathbb{H}$ linear operator $\tilde{X}: D(\widetilde{X}) \rightarrow \mathrm{H}$ such that $J \widetilde{X} \subset \widetilde{X} J$ and $\widetilde{X}_{J}=X$ where in particular 
$D(X)=D(\widetilde{X}) \cap \mathrm{H}_{J}$.

(c) If $X$ is as in (b) and $S: D(S) \rightarrow \mathrm{H}_{J}$, with $D(S) \subset \mathrm{H}_{J}$, is another $\mathbb{C}_{j}$-linear operator, the following facts are true on the relevant natural domains.

(i) $\widetilde{X} J=J \widetilde{X}$.

(ii) $\widetilde{a X}=a \widetilde{X}$ for every $a \in \mathbb{R}$.

(iii) $\widetilde{S+T}=\widetilde{S}+\widetilde{T}$.

(iv) $\widetilde{S T}=\widetilde{S} \widetilde{T}$.

(v) $\widetilde{S} \subset \widetilde{T}$ iff $S \subset T$.

(vi) $X \in \mathfrak{B}\left(\mathrm{H}_{J}\right)$ if and only if $\widetilde{X} \in \mathfrak{B}(\mathrm{H})$. In this case $\|\tilde{X}\|=\|X\|$.

(vii) $D(\widetilde{X})$ is dense in $\mathrm{H}$ if and only if $D(X)$ is dense in $\mathrm{H}_{J}$.

(viii) $(\widetilde{X})^{*}=\widetilde{X}^{*}$ if $X$ is densely defined.

As a consequence $X$ is symmetric, essentially selfadjoint, selfadjoint, antiselfadjoint, isometric, unitary, idempotent if and only if $\widetilde{X}$ is, respectively, symmetric, essentially selfadjoint, selfadjoint, antiselfadjoint, isometric, unitary, idempotent on $\mathrm{H}$.

(ix) $X$ is closable if and only if $\tilde{X}$ is closable. In this case $\widetilde{\bar{X}}=\overline{\widetilde{X}}$.

(d) If $\mathfrak{B}(\mathrm{H}) \ni A_{n} \rightarrow A \in \mathfrak{B}(\mathrm{H})$ weakly, resp., strongly for $n \rightarrow+\infty$ and $J A_{n}=A_{n} J$, then $J A=A J$ and $\mathfrak{B}\left(\mathrm{H}_{J}\right) \ni\left(A_{n}\right)_{J} \rightarrow A_{J} \in \mathfrak{B}\left(\mathrm{H}_{J}\right)$ weakly, resp., strongly for $n \rightarrow+\infty$.

The stated results extends into the following result which consider constructions related to spectral measures.

Proposition 3.5 Let $\mathrm{H}$ be a quaternionic Hilbert space, $J \in \mathfrak{B}(\mathrm{H})$ a complex structure and $A: D(A) \rightarrow \mathrm{H}$, where $D(A) \subset \mathrm{H}$, a selfadjoint $\mathbb{H}$-linear operator such that $J A \subset A J$. Then the following facts hold referring to the notation in (b) Prop 3.4.

(a) If $P^{(A)}$ is the PVM of the spectral decomposition of $A$, the operators $\left(P_{E}^{(A)}\right)_{J}$ with $E \subset \mathbb{R}$ Borel set, form the PVM $P^{\left(A_{J}\right)}$ of $A_{J}$ in $\mathrm{H}_{J}$. Furthermore the map

$$
P^{(A)} \ni P_{E}^{(A)} \mapsto\left(P_{E}^{(A)}\right)_{J} \in P^{\left(A_{J}\right)}
$$

is an isomorphism of $\sigma$-complete Boolean lattices.

(b) If $f: \mathbb{R} \rightarrow \mathbb{R}$ is Borel measurable, then $f(A) J=J f(A)$ and $f(A)_{J}=f\left(A_{J}\right)$.

(c) $A \geq 0$ if and only if $A_{J} \geq 0$.

(d) $\sigma_{S}(A)=\sigma\left(A_{J}\right), \sigma_{p S}(A)=\sigma_{p}\left(A_{J}\right), \sigma_{c S}(A)=\sigma_{c}\left(A_{J}\right)$, where $\sigma_{S}$ denotes the spherical spectrum of the quaternionic linear operator $A$ [11, 13].

Proof. See Appendix A. 
To conclude, let us examine the interplay of the von Neumann algebra structure in $\mathrm{H}$ and that in $\mathrm{H}_{J}$.

Proposition 3.6 Let $\mathfrak{R}$ be a von Neumann algebra over the quaternionic Hilbert space $\mathrm{H}$ and $J \in \mathfrak{R}^{\prime} \cap \mathfrak{R}$ be a complex structure. Then $\mathfrak{R}_{J}:=\left\{A_{J} \mid A \in \mathfrak{R}\right\}$ is a von Neumann algebra over the $\mathbb{C}_{j}$-complex Hilbert space $\mathrm{H}_{J}$.

Proof. $\mathfrak{R}_{J}$ is a unital ${ }^{*}$-subalgebra of $\mathfrak{B}\left(\mathrm{H}_{J}\right)$, indeed $I \in \mathfrak{R}_{J}$ and if $A_{J}, B_{J} \in \mathfrak{R}_{J}$, then $A_{J}+B_{J}=(A+B)_{J}$ belongs to $\mathfrak{R}_{J}$. If furthermore $a+j b \in \mathbb{C}_{j}$, since $J \in \mathfrak{R}$, we have $J_{J} \in \mathfrak{R}_{J}$ and $(a+j b) A_{J}:=a A_{J}+b J_{J} A_{J}=(a A+b J A)_{J}$ is a well defined $\mathbb{C}_{j}$-linear operator in $\mathfrak{R}_{J}$. Finally, if $A_{J} \in \mathfrak{R}_{J}$ then $\left(A_{J}\right)^{*}=\left(A^{*}\right)_{J} \in \mathfrak{R}_{J}$. These properties ensures that $\mathfrak{R}_{J}$ is a unital *-subalgebra of $\mathfrak{B}\left(\mathrm{H}_{J}\right)$ as said above. To conclude it is enough establishing that $\mathfrak{R}_{J}$ is strongly closed. Let $\left(C_{n}\right)_{n \in \mathbb{N}} \subset \mathfrak{R}_{J}$ which strongly converges to some $C \in \mathfrak{B}\left(\mathrm{H}_{J}\right)$, we want to prove that $C \in \mathfrak{R}_{J}$. By definition, $C_{n}=\left(K_{n}\right)_{J}$ for $K_{n} \in \mathfrak{R}, C=K_{J}$ for some $K \in \mathfrak{B}(\mathrm{H})$, and $\left\|K_{n} u-K u\right\| \rightarrow 0$ for every $u \in \mathrm{H}_{J}$. So, take $x \in \mathrm{H}$, we know from Prop 3.3 that $x=x_{1}+x_{2} k$ for unique $x_{1}, x_{2} \in \mathrm{H}_{J}$. Hence, again for Prop 3.3. $\left\|K_{n} x-K x\right\|^{2}=$ $\left\|\left(K_{n} x_{1}-K x_{1}\right)+\left(K_{n} x_{2}-K x_{2}\right) k\right\|^{2}=\left\|\left(K_{n} x_{1}-K x_{1}\right)\right\|^{2}+\left\|K_{n} x_{2}-K x_{2}\right\|^{2} \rightarrow 0$. All that proves that $\mathfrak{R} \ni K_{n} \rightarrow K$ strongly and thus $K \in \mathfrak{R}$ because it is a von Neumann algebra. Summing up, $\mathfrak{R}_{J} \ni C_{n} \rightarrow C=K_{J} \in \mathfrak{R}_{J}$ s that $\mathfrak{R}_{J}$ is strongly closed and therefore is a von Neumann algebra.

\subsection{Restriction to a real Hilbert subspace induced by two anticommuting complex struc- tures}

If $\mathrm{H}$ is a quaternionic Hilbert space, suppose we have a pair $J, K$ of anticommuting $(J K=-K J)$ complex structures. We want to prove that the set

$$
\mathrm{H}_{J K}:=\{u \in \mathrm{H} \mid J u=u j, K u=u k\}
$$

is a real Hilbert space. $\mathrm{H}_{J K}$ is evidently a real subspace of $\mathrm{H}$ which also satisfies $J K u=u i$ if $u \in H_{J K}$ from $i=j k$. However it is not obvious that the scalar product of $\mathrm{H}$ makes $\mathrm{H}_{J K}$ a real Hilbert space. To prove it, we introduce an important technical tool given by the map

$$
L: \mathbb{H} \ni q \mapsto L_{q}:=a I+b J K+c J+d K \in \mathfrak{B}(\mathrm{H}) \quad \text { where } q=a+i b+j c+k d .
$$

$L$ is a left multiplication ([11, 13]) on $\mathrm{H}$ - an injective unital real *-algebra homomorphism - associated to $J$ and $K$. Notice that, exploiting definitions (13) and (14), it holds

$$
L_{q} u=u q \quad \forall q \in \mathbb{H}
$$

In this sense the elements of $\mathrm{H}_{J K}$ can be interpreted as real vectors of $\mathrm{H}$, as they commute with every element of $\mathbb{H}$. 
Proposition 3.7 Referring to (13) a 7 nd (14), there exists a Hilbert basis $N$ of $\mathrm{H}$ such that $N \subset \mathrm{H}_{J K}$ and

$$
L_{q}=\sum_{z \in N} z q\langle z \mid \cdot\rangle, \quad \forall q \in \mathbb{H}
$$

so that $L_{q} z=z q$ for every $z \in N$ in particular.

Proof. The proof immediately arises from Thm 4.3 in [13].

We are in a position to state and prove the result afore mentioned. Notice that, with obvious notation, the sets $\mathrm{H}_{J K} i, \mathrm{H}_{J K} j$, and $\mathrm{H}_{J K} k$ are real subspaces of $\mathrm{H}$.

Proposition 3.8 Let $\mathrm{H}$ be a quaternionic Hilbert space and $J, K \in \mathfrak{B}(\mathrm{H})$ two anticommuting complex structures. The real vector subspace $\mathrm{H}_{J K}$ defined in (13) equipped with the restriction $\langle\cdot \mid \cdot\rangle_{J K}$ of the scalar product of $\mathrm{H}$ to $\mathrm{H}_{J K}$ satisfies the following properties.

(a) $\mathrm{H}_{J K}$ is a real Hilbert space, non-trivial unless $\mathrm{H}=\{0\}$.

(b) The direct (generally not orthogonal) decomposition is valid

$$
\mathrm{H}=\mathrm{H}_{J K} \oplus \mathrm{H}_{J K} i \oplus \mathrm{H}_{J K} j \oplus \mathrm{H}_{J K} k
$$

such that

$$
\|x\|^{2}=\sum_{i=1}^{4}\left\|x_{i}\right\|^{2} \quad \text { if } \mathrm{H} \ni x=x_{1}+x_{2} i+x_{3} j+x_{a} k \text { with } x_{1}, x_{2}, x_{3}, x_{4} \in \mathrm{H}_{J K}
$$

(c) The maps

$$
\begin{aligned}
& \mathrm{H}_{J K} \ni u \mapsto u i \in \mathrm{H}_{J K} i, \\
& \mathrm{H}_{J K} \ni u \mapsto u j \in \mathrm{H}_{J K} j, \\
& \mathrm{H}_{J K} \ni u \mapsto u k \in \mathrm{H}_{J K} k
\end{aligned}
$$

are $\mathbb{R}$-linear, isometric and bijective.

(d) If $N \subset \mathrm{H}_{J K}$ is a Hilbert basis of $\mathrm{H}_{J K}$, then $N$ is also a Hilbert basis of $\mathrm{H}$.

Proof. (a) Let us prove that $\langle\cdot \mid \cdot\rangle$ takes values in $\mathbb{R}$ if restricted to $\mathrm{H}_{J K}$. Let $u, v \in \mathrm{H}_{J K}$ and $q \in \mathbb{H}$, exploiting (15) we get

$$
\langle u \mid v\rangle q=\langle u \mid v q\rangle=\left\langle u \mid L_{q} v\right\rangle=\left\langle L_{q}^{*} u \mid v\right\rangle=\left\langle L_{\bar{q}} u \mid v\right\rangle=\langle u \bar{q} \mid v\rangle=q\langle u \mid v\rangle
$$

Since $q$ is generic, $\langle u \mid v\rangle$ must be real. $\langle\cdot \mid \cdot\rangle$ restricted to $\mathrm{H}_{J K}$ is therefore a real scalar product denoted by $\langle\cdot \mid \cdot\rangle_{J K}$, because the remaining properties immediately arises from those valid in $\mathrm{H}$. As aconsequence, the norm induced by $\langle\cdot \mid \cdot\rangle_{J K}$ is equal to the norm of $\mathrm{H}$ restricted to $\mathrm{H}_{J K}$. As $\mathrm{H}_{J K}$ is closed because $J$ and $K$ are continuous, it is complete as a normed space, since $\mathrm{H}$ is, and thus it is a real Hilbert space. It is also obvious that $N$ of Prop. 3.7 is a Hilbert basis for $\mathrm{H}_{J K}$ because it is an orthonormal subset of $\mathrm{H}_{J K}$ whose orthogonal is $\{0\}$. Since $N$ is non empty if $\mathrm{H}$ is non-trivial, $\mathrm{H}_{J K} \neq\{0\}$.

(b) The last statement in (b) arises immediately bearing in mind that when computing $\|x\|^{2}=\langle x \mid x\rangle$ exploiting the decomposition $x=x_{1}+x_{2} i+x_{3} j+x_{4} k$ it holds $\left\langle x_{a} \mid x_{b}\right\rangle=$ 
$\left\langle x_{b} \mid x_{a}\right\rangle \in \mathbb{R}$, because $x_{a}, x_{b} \in \mathrm{H}_{J K}$, and this impleis that the mixed terms in the expansion of the scalar product $\langle x \mid x\rangle$ cancel pairwise. Now, let us prove the first part. Let $u \in \mathrm{H}$, then a direct inspection shows that $u_{1}:=u-J K u i-J u j-K u k \in \mathrm{H}_{J K}, u_{j k}:=u-J K u i+$ $J u j+K u k \in\left(\mathrm{H}_{J K}\right) i, u_{j}:=u+J K u i-J u j+K u k \in\left(\mathrm{H}_{J K}\right) j$, and $u_{k}:=u+J K u i+J u j-$ $K u k \in\left(\mathrm{H}_{J K}\right) k$. Summing together the four identities we obtain $4 u=u_{1}+u_{i}+u_{j}+u_{k}$. The found decomposition is unique, and thus the decomposition of $\mathrm{H}$ is direct. Indeed suppose that $x_{1}+x_{2} i+x_{3} j+x_{4} j k$ and $x_{1}^{\prime}+x_{2}^{\prime} i+x_{3}^{\prime} j+x_{4}^{\prime} j k$ are two decompositions of the same vector $x \in \mathrm{H}$, then $\left(x_{1}-x_{1}^{\prime}\right)+\left(x_{2}-x_{2}^{\prime}\right) i+\left(x_{3}-x_{3}^{\prime}\right) j+\left(x_{4}-x_{4}^{\prime}\right) j k=0$. Exploiting (16) we immediately get $\left\|x_{\alpha}-x_{\alpha}^{\prime}\right\|=0$, hence $x_{\alpha}=x_{\alpha}^{\prime}$, for all $\alpha=1,2,3,4$ concluding the proof.

(c) The proof is elementary using (b) and the basic properties of $J$ and $K$. The proof of (d) easily arises from the first part of (b) and the fact that $\langle x \mid y\rangle \in \mathbb{R}$ if $x, y \in \mathrm{H}_{J K}$.

We can now pass to deal with operators and their restrictions to $\mathrm{H}_{J K}$.

Proposition 3.9 With the same hypotheses as in Proposition 3.8 and with $L$ as in (14), the following facts hold.

(a) Consider a $\mathbb{H}$-linear operator $X: D(X) \rightarrow \mathrm{H}$ where $D(X) \subset \mathrm{H}$, such that $J X \subset X J$ and $K X \subset X K$. Then $X_{J K}:=\left.X\right|_{D(X) \cap \mathrm{H}_{J K}}$ is a well defined $\mathbb{R}$-linear operator in $\mathrm{H}_{J K}$.

(b) Consider an $\mathbb{R}$-linear operator $A: D(A) \rightarrow \mathrm{H}_{J K}$, where $D(A) \subset \mathrm{H}_{J K}$. Then there exists a unique $\mathbb{H}$-linear operator $\tilde{A}: D(\widetilde{A}) \rightarrow \mathrm{H}$, with $D(\widetilde{A}) \subset \mathrm{H}$, such that $J \widetilde{A} \subset \widetilde{A} J$, $K \widetilde{A} \subset \widetilde{A} K$, and $A=\widetilde{A}_{J K}$, in particular $D(A)=D(\widetilde{A}) \cap \mathrm{H}_{J K}$.

(c) The following facts hold if $A$ is as in (b) and $B: D(B) \rightarrow \mathrm{H}_{J K}$ is another $\mathbb{R}$-linear operator where $D(B) \subset \mathrm{H}_{J K}$.

(i) $L_{q} \widetilde{A}=\widetilde{A} L_{q}$ for all $q \in \mathbb{H}$.

(ii) $\widetilde{a A}=a \widetilde{A}$ for every $a \in \mathbb{R}$.

(iii) $\widetilde{A+B}=\widetilde{A}+\widetilde{B}$.

(iv) $\widetilde{A B}=\widetilde{A} \widetilde{B}$.

(v) $A \subset B$ iff $\tilde{A} \subset \widetilde{B}$.

(vi) $A \in \mathfrak{B}\left(\mathrm{H}_{J K}\right)$ iff $\widetilde{A} \in \mathfrak{B}(\mathrm{H})$ and $\|\widetilde{A}\|=\|A\|$.

(vii) $D(A)$ is dense if and only if $D(\tilde{A})$ is dense

(viii) $\widetilde{A^{*}}=(\widetilde{A})^{*}$ if $A$ is densely defined

As a consequence, $A$ is symmetric, antisymmetric, (essentially) selfadjoint, antiselfadjoint, unitary, idempotent iff $\widetilde{A}$ is symmetric, antisymmetric, (essentially) selfadjoint, antiselfadjoint, unitary, idempotent

(ix) $A$ is closable iff $\widetilde{A}$ is closable. In this case $\overline{\widetilde{A}}=\widetilde{\bar{A}}$.

(d) If $\mathfrak{B}(\mathrm{H}) \ni X_{n} \rightarrow X \in \mathfrak{B}(\mathrm{H})$ weakly, resp., strongly for $n \rightarrow+\infty$ and $J X_{n}=X_{n} J$, and $K X_{n}=X_{n} K$, then $J X=X J, K X=X K$ and $\mathfrak{B}\left(\mathrm{H}_{J K}\right) \ni\left(A_{n}\right)_{J K} \rightarrow A_{J K} \in \mathfrak{B}\left(\mathrm{H}_{J K}\right)$ 
weakly, resp., strongly for $n \rightarrow+\infty$.

Proof. See Appendix A

Proposition 3.10 Let $\mathrm{H}$ be a quaternionic Hilbert space and $J, K \in \mathfrak{B}(\mathrm{H})$ two anticommuting complex structures. If $A: D(A) \rightarrow \mathrm{H}$, where $D(A) \subset \mathrm{H}$, is selfadjoint and satisfy $J A \subset A J$ and $K A \subset A K$, then the following facts hold true.

(a) If $P^{(A)}$ is the PVM of the spectral decomposition of $A$, the operators $\left(P_{E}^{(A)}\right)_{J K}$ with $E \subset \mathbb{R}$ Borel set, form the PVM $P^{\left(A_{J K}\right)}$ of $A_{J K}$ in $\mathrm{H}_{J K}$. Furthermore the map

$$
P^{(A)} \ni P_{E}^{(A)} \mapsto\left(P_{E}^{(A)}\right)_{J K} \in P^{\left(A_{J K}\right)}
$$

is an isomorphism of $\sigma$-complete Boolean lattices.

(b) if $f: \mathbb{R} \rightarrow \mathbb{R}$ is Borel measurable function, then $f(A) J=J f(A)$ and $f(A) K=K f(A)$ and $f(A)_{J K}=f\left(A_{J K}\right)$.

(c) $A$ is positive if and only if $A_{J K}$ is positive.

(d) $\sigma_{S}(A)=\sigma\left(A_{J K}\right), \sigma_{p S}(A)=\sigma_{p}\left(A_{J K}\right), \sigma_{c S}(A)=\sigma_{c}\left(A_{J K}\right)$.

Proof. The proof is analogous to the one carried out for Proposition 3.5.

As we did for the complex case, we can now prove the following result.

Proposition 3.11 Let $\mathfrak{R}$ a von Neumann algebra over a quaternionic Hilbert space $\mathrm{H}$ and $J, K \in \mathfrak{R}^{\prime}$ be complex structures such that $J K=-K J$. Then $\mathfrak{R}_{J K}:=\left\{A_{J K} \mid A \in \mathfrak{R}\right\}$ is a von Neumann algebra over $\mathrm{H}_{J K}$.

Proof. $\mathfrak{R}_{J K}$ is clearly a unital sub ${ }^{*}$-algebra of $\mathfrak{B}\left(\mathrm{H}_{J K}\right)$ thanks to Prop. 3.9 and the fact that only real combinations are to be taken into account in the algebra $\mathfrak{B}\left(\mathrm{H}_{J K}\right)$ as well as in the algebra $\mathfrak{B}(\mathrm{H})$. The algebra $\mathfrak{R}_{J K}$ turns out to be closed in the strong topology on $\mathrm{H}_{J K}$, the proof being identical to the one carried out in Prop. 3.6.

\section{Lie-Group representations in quaternionic Hilbert spaces}

This section is devoted to generalize to the quaternionic Hilbert space case some wellknown results of representation theory especially of Lie groups in terms of unitary operators.

\subsection{Gårding domain and Lie algebra representation}

As usual, $C_{0}^{\infty}(G)$ denotes the vector space of real-valued infinitely differentiable compactly supported functions on a given (real finite-dimensional) Lie group $G$ and we consider a 
strongly-continuous unitary representation $U$ of $G$ on a quaternionic Hilbert space $\mathrm{H}$. If $x \in \mathrm{H}$ and $f \in C_{0}^{\infty}(G)$ we define

$$
x[f]=\int_{G} f(g) U_{g} x d g
$$

where $d g$ is the left-invariant Haar measure on $G$, as the unique vector in $\mathrm{H}$ such that $\langle y \mid x[f]\rangle=\int_{G} f(g)\left\langle y \mid U_{g} x\right\rangle d g$ for all $y \in \mathrm{H}$ - the existence and uniqueness of such a vector is the content of the Riesz representation theorem which holds true also on quaternionic Hilbert space, as described in [11].

Next we may extend to the quaternionic Hilbert space case a well-known definition of the real and complex cases (see, e.g., [23] for the real case).

Definition 4.1 Given a Lie group $G$ and a strongly-continuous unitary representation $U$ of $G$ on a quaternionic Hilbert space $\mathbf{H}$, the $\mathbb{H}$-linear subspace of $\mathrm{H}$ generated by all the vectors $x[f]$ (17) is called the Gårding Domain associated with $U$ and denoted by $\mathcal{D}_{G}^{(U)}$.

Remark 4.2 Actually $\mathcal{D}_{G}^{(U)}$ coincides with the real span of vectors $x[f]$. Indeed,

$$
\begin{aligned}
& \langle y \mid x[f] q\rangle=\langle y \mid x[f]\rangle q=\left(\int_{G} f(g)\left\langle y \mid U_{g} x\right\rangle d g\right) q=\int_{G} f(g)\left\langle y \mid U_{g} x\right\rangle q d g= \\
& =\int_{G} f(g)\left\langle y \mid\left(U_{g} x\right) q\right\rangle d g=\int_{G} f(g)\left\langle y \mid U_{g}(x q)\right\rangle q d g=\langle y \mid(x q)[f]\rangle
\end{aligned}
$$

Since this holds for any $y \in \mathbf{H}$ we have that $x[f] q=(x q)[f]$.

We can immediately prove the following result. Due to (d) below, the elements of $D_{G}^{(U)}$ are also called the smooth vectors of $U$.

Proposition 4.3 Referring to Def[4.1, the following facts hold true.

(a) Denoting by $U_{\mathbb{R}}$ and $U_{\mathbb{C}_{j}}$ the map $U$ respectively viewed as strongly-continuous unitary representations on $\mathrm{H}_{\mathbb{R}}$ and $\mathrm{H}_{\mathbb{C}_{j}}$, it holds $\mathcal{D}_{G}^{(U)}=\mathcal{D}_{G}^{\left(U_{\mathbb{R}}\right)}=\mathcal{D}_{G}^{\left(U_{\mathbb{C}_{j}}\right)}$

(b) $\mathcal{D}_{G}^{(U)}$ is dense in $\mathrm{H}$.

(c) $U_{g}\left(\mathcal{D}_{G}^{(U)}\right) \subset \mathcal{D}_{G}^{(U)}$ for all $g \in G$.

(d) $x \in D_{G}^{(U)}$ if and only if the map $G \ni g \mapsto U_{g} x$ is smooth at every point $g \in G$ with respect to the smooth atlas of $G$.

Proof. We prove (a) and (d). Items (b) and (c) immediately follow from (a) and the Gårding theory on real Hilbert spaces (e.g., see [23] Thm 3.6). Let $x \in \mathrm{H}_{\mathbb{R}}=\mathrm{H}$ and $f \in C_{0}^{\infty}(G), x(f)$ is the vector (17) but defined with respect to the real Hilbert space structure of $\mathrm{H}_{\mathbb{R}}$ while we keep the notation $x[f]$ for the quaternionic case. We intend to 
prove that $x(f)=x[f] . x(f)$ is the only vector such that $(y \mid x(f))=\int_{G} f(g)\left(y \mid U_{g} x\right) d g$ for all $y \in \mathrm{H}_{\mathbb{R}}$, where $(\cdot \mid \cdot)=R e\langle\cdot \mid \cdot\rangle . x[f]$, instead, is the only vector such that $\langle y \mid x[f]\rangle=$ $\int_{G} f(g)\left\langle y \mid U_{g} x\right\rangle d g$ for all $y \in \mathrm{H}$. Taking the real part of both sides we get $R e\langle y \mid x[f]\rangle=$ $\int_{G} f(g) \operatorname{Re}\left\langle y \mid U_{g} x\right\rangle d g$ for all $y \in \mathrm{H}=\mathrm{H}_{\mathbb{R}}$. By definition of $x(f)$ it follows $x[f]=x(f)$. This clearly proves that $\mathcal{D}_{G}^{\left(U_{\mathbb{R}}\right)} \subset \mathcal{D}_{G}^{(U)}$. The converse inclusion holds because $(x[f]) q=(x q)[f]$ for every $q \in \mathbb{H}$ and thus $x[f] j, x[f] k, x[f] j k$ belongs to $\mathcal{D}_{G}^{\left(U_{\mathbb{R}}\right)}$. We have established that $\mathcal{D}_{G}^{\left(U_{\mathbb{R}}\right)}=\mathcal{D}_{G}^{(U)}$. Since $\mathrm{H}_{\mathbb{C}_{j}}=\left(\mathrm{H}_{\mathbb{R}}\right)_{\mathcal{J}}$, (b) Thm 3.5 in [23] proves $\mathcal{D}_{G}^{\left(U_{\mathbb{R}}\right)}=\mathcal{D}_{G}^{\left(U_{\mathbb{C}_{j}}\right)}$.

(d) The notion of differentiability only uses the norm and $\mathbb{R}$-linearity of $\mathrm{H}$, hence $G \ni g \mapsto$ $U_{g} x$ is smooth with respect to $\mathrm{H}$ if and only if is smooth with respect to the underlying real structure $H_{\mathbb{R}}$. Since the thesis holds for real Hilbert spaces, point (a) concludes the proof.

Let us pass to the representation theory of the the Lie algebra $\mathfrak{g}$ associated to a Lie group $G$. Take $\mathbf{A} \in \mathfrak{g}$ and consider the one-parameter subgroup $\mathbb{R} \ni t \mapsto \exp (t \mathbf{A}) \in G$. Thanks to Theorem 2.13 there exists a unique antiselfadjoint operator $A$ on $\mathrm{H}$ such that $U_{\exp (t \mathbf{A})}=e^{t A}$ and $A$ equals the antiselfadjoint generators of $t \mapsto U_{\exp (t \mathbf{A})}$ when interpreted as acting on $\mathrm{H}_{\mathbb{R}}$ or $\mathrm{H}_{\mathbb{C}_{j}}$. Thanks to Prop 4.3 (a) and the properties of Gårding domain in real Hilbert spaces, we easily get the following result.

Proposition 4.4 Consider a Lie group $G$ with Lie algebra $\mathfrak{g}$, and a strongly-continuous unitary representation $U$ of $G$ on a quaternionic Hilbert space $\mathrm{H}$. The following facts are valid.

(a) If $\mathbf{A} \in \mathfrak{g}$ and $U_{\exp (t \mathbf{A})}=e^{t A}$, then $A\left(\mathcal{D}_{G}^{(U)}\right) \subset \mathcal{D}_{G}^{(U)}$ and $\mathcal{D}_{G}^{(U)}$ is a core for $A$, i.e., $\overline{\left.A\right|_{\mathcal{D}_{G}^{(U)}}}=A$.

(b) The map $\mathfrak{g} \ni \mathbf{A} \mapsto u(\mathbf{A}):=\left.A\right|_{\mathcal{D}_{G}^{(U)}} \in \mathcal{L}\left(\mathcal{D}_{G}^{(U)}\right)$ is a Lie algebra homomorphism.

(c) If $u_{\mathbb{R}}$ and $u_{\mathbb{C}_{j}}$ denotes the Lie algebra homomorphism associated with $U$ as above in $\mathrm{H}_{\mathbb{R}}$ and $\mathrm{H}_{\mathbb{C}_{j}}$ respectively, it holds $u(\mathbf{A})=u_{\mathbb{R}}(\mathbf{A})=u_{\mathbb{C}_{j}}(\mathbf{A})$. In particular the antiselfadjoint generators related to $\mathbf{A}$ defined on $\mathrm{H}, \mathrm{H}_{\mathbb{R}}, \mathrm{H}_{\mathbb{C}_{j}}$ are equal.

Proof. The antiselfadjoint generators related to $\mathbf{A}$ defined on $\mathrm{H}, \mathrm{H}_{\mathbb{R}}, \mathrm{H}_{\mathbb{C}}$ are equal due to Thm2.13. Since also $\mathcal{D}_{G}^{(U)}$ is independent from the Hilbert space (Prop 4.3), exploiting Th 3.6 (c) in [23] we get $A\left(\mathcal{D}_{G}^{(U)}\right) \subset \mathcal{D}_{G}^{(U)}$ and thus $u$ is independent as well. We have proved (c). Now, let $\mathcal{L}_{\mathbb{R}}\left(\mathcal{D}_{G}^{(U)}\right)$ be the Lie algebra of real-linear operators defined on $\mathcal{D}_{G}^{(U)}$, of course $\mathcal{L}\left(\mathcal{D}_{G}^{(U)}\right) \subset \mathcal{L}_{\mathbb{R}}\left(\mathcal{D}_{G}^{(U)}\right)$. The map

$$
\mathfrak{g} \ni \mathbf{A} \mapsto u(\mathbf{A}):=\left.A\right|_{\mathcal{D}_{G}^{(U)}} \in \mathcal{L}_{\mathbb{R}}\left(\mathcal{D}_{G}^{(U)}\right)
$$

is a Lie algebra homomorphism thanks to Thm $3.6(\mathrm{~d})$ in [23] applied to $\mathrm{H}_{\mathbb{R}}$. Since actually $\left.A\right|_{\mathcal{D}_{G}^{(U)}} \in \mathcal{L}\left(\mathcal{D}_{G}^{(U)}\right)$, (b) is true. Finally, exploiting Thm (f) 3.6 in [23] and (e)-(v) Prop. 2.1 we conclude the proof of (a). 
As we did in the real theory, we can consider the real associative unital universal enveloping algebra $E_{\mathfrak{g}}$ of the Lie algebra $\mathfrak{g}$ (e.g., see [39] and the quick account in Appendix E of [23]). By construction $\mathfrak{g} \subset E_{\mathfrak{g}}$ as Lie subalgebra and, if o denotes the product of the algebra whch extends that of $\mathfrak{g}$, a generic element of $E_{\mathfrak{g}}$ takes the form

$$
\mathbf{M}=c_{0} 1+\sum_{k=1}^{N} \sum_{j=1}^{N_{k}} c_{j k} \mathbf{A}_{j 1} \circ \ldots \circ \mathbf{A}_{j k}
$$

for some $N, N_{k} \in \mathbb{N}, c_{0}, c_{j k} \in \mathbb{R}$ and $\mathbf{A}_{j m} \in \mathfrak{g}$. We also assume to endow $E_{\mathfrak{g}}$ with the standard real involution ${ }^{+}$completely defined by requiring

$$
\left(c_{0} 1+\sum_{k=1}^{N} \sum_{j=1}^{N_{k}} c_{j k} \mathbf{A}_{j 1} \circ \ldots \circ \mathbf{A}_{j k}\right)^{+}=c_{0} 1+\sum_{k=1}^{N} \sum_{j=1}^{N_{k}} c_{j k}(-1)^{k} \mathbf{A}_{j k} \circ \ldots \circ \mathbf{A}_{j 1} .
$$

An element $\mathbf{M} \in \mathfrak{g}$ is said to be symmetric if $\mathbf{M}^{+}=\mathbf{M}$, so that $\mathfrak{g}$ is made of antisymmetric elements. An important technical role is played by the Nelson elements of $E_{\mathfrak{g}}$ which are those of the form

$$
\mathbf{N}:=\sum_{i=1}^{n} \mathbf{X}_{i} \circ \mathbf{X}_{i}
$$

where $\left\{\mathbf{X}_{1}, \ldots \mathbf{X}_{n}\right\}$ is any basis of $\mathfrak{g}$. Notice that they are symmetric by construction. Exactly as above we can now take advantage of the real theory and state the following theorem which has the same proof as the corresponding items of Thm 3.6 and Prop.3.8 in $[23]$.

Theorem 4.5 Referring to Proposition 4.4 the following facts hold

1. The Lie algebra homomorphism $u$ defined in (18) uniquely extends to a real unital associative algebra representation of the universal enveloping algebra $E_{\mathfrak{g}}$ defined on $\mathcal{D}_{G}^{(U)}$. More precisely, if $\mathbf{M}$ is taken as in (19), then it holds

$$
u(\mathbf{M})=\left.c_{0} I\right|_{\mathcal{D}_{G}^{(U)}}+\sum_{k=1}^{N} \sum_{j=1}^{N_{k}} c_{j k} u\left(\mathbf{A}_{j 1}\right) \cdots u\left(\mathbf{A}_{j k}\right)
$$

It also holds $u\left(\mathbf{M}^{+}\right) \subset u(\mathbf{M})^{*}$, in particular $u(\mathbf{M})$ is symmetric if $\mathbf{M}^{+}=\mathbf{M}$.

2. Suppose that $\mathbf{M} \in E_{\mathfrak{g}}$ satisfies both $\mathbf{M}=\mathbf{M}^{+}$and $[\mathbf{M}, \mathbf{N}]_{\mathfrak{g}}=0$ for some Nelson element $\mathbf{N}$, then $u(\mathbf{M})$ is essentially self-adjoint. In particular $u(\mathbf{N})$ is always essentially self-adjoint.

3. if $B \in \mathfrak{B}(\mathrm{H})$ the following facts are equivalent

(i) $B u(\mathbf{A}) \subset u(\mathbf{A}) B$ for every $\mathbf{A} \in \mathfrak{g}$ 
(ii) $B \overline{u(\mathbf{A})} \subset \overline{u(\mathbf{A})} B$ for every $\mathbf{A} \in \mathfrak{g}$

(iii) $B U_{g}=U_{g} B$ for every $g \in G$

If one of these conditions is satisfies, then $B\left(\mathcal{D}_{G}^{(U)}\right) \subset \mathcal{D}_{G}^{(U)}$

\subsection{Analytic vectors of unitary representations in quaternionic Hilbert spaces}

There is another important subspace of $\mathrm{H}$ made of "good vectors" for a strongly-continuous unitary representation $U$ of a Lie group $G$, even better than $\mathcal{D}_{G}^{(U)}$. To introduce this space we need a definition. A function $f: \mathbb{R}^{n} \supset U \rightarrow \mathrm{H}$ is called real analytic at $x_{0} \in U$ if there exists a neighbourhood $V \subset U$ of $x_{0}$ where the function $f$ can be expanded in power series

$$
f(x)=\sum_{|\alpha|=n, n=0}^{\infty}\left(x-x_{0}\right)^{\alpha} v_{\alpha}, x \in V
$$

for suitable $v_{\alpha} \in \mathrm{H}$ for every multi-index $\alpha \in \mathbb{N}^{n}$.

Definition 4.6 Let $\mathrm{H}$ be a quaternionic Hilbert space and $G \ni g \mapsto U_{g}$ a unitary strongly-continuous representation on $\mathrm{H}$ of the Lie group $G$. A vector $x \in \mathrm{H}$ is said to be analytic for $U$ if the function $g \mapsto U_{g} x$ is real analytic at every point $g \in G$, referring to the analytic atlas of $G$. The linear subspace of $H$ made of these vectors is called the Nelson space of the representation and denoted by $\mathcal{D}_{N}^{(U)}$.

Remark 4.7 Adopting notations as in Prop 4.3, it is evident from the definition that $\mathcal{D}_{N}^{(U)}=\mathcal{D}_{N}^{\left(U_{\mathbb{R}}\right)}=\mathcal{D}_{N}^{(U)}$

There exists another related definition of analyticity for vectors. Let $A: D(A) \rightarrow \mathrm{H}$ an operator in a quaternionic Hilbert space $\mathbf{H}$, we say that a vector $x \in \bigcap_{n=0}^{+\infty} D\left(A^{n}\right)$ is analytic for $A$, if there exists $t_{x}>0$ such that

$$
\sum_{n=0}^{+\infty} \frac{t_{x}^{n}}{n !}\left\|A^{n} x\right\|<+\infty
$$

From the elementary theory of series of powers, we know that $t$ above can be replaced for every $z \in \mathbb{C}$ with $|z|<t_{x}$ obtaining an absolutely convergent series.

Remark 4.8 It should be evident that the analytic vectors for $A$ form a subspace of $D(A)$. Moreover a vector $x$ is analytic for $A$ on $\mathrm{H}$ if and only if it is analytic for $A$ on $\mathrm{H}_{\mathbb{R}}$ if and only if it is analytic for $A$ on $\mathrm{H}_{\mathbb{C}_{j}}$

One of remarkable Nelson's results, here extended to quaternionic Hilbert spaces, states that

Proposition 4.9 Consider an operator $A: D(A) \rightarrow \mathrm{H}$ on a quaternionic Hilbert space $\mathrm{H}$. 
(a) If $A$ is anti selfadjoint and $x \in D(A)$ is analytic with $t_{x}>0$ as in (24), then

$$
e^{t A} x=\sum_{n=0}^{+\infty} \frac{t^{n}}{n !} A^{n} x \quad \text { if } t \in \mathbb{R} \text { satisfies }|t| \leq t_{x} .
$$

(b) If $A$ is (anti) symmetric and $D(A)$ includes a set of analytic vectors whose finite span is dense in $\mathrm{H}$, then $\bar{A}$ is (anti) selfadjoint and $D(A)$.

Proof. Point (a). Since the thesis holds for real Hilbert spaces (Thm 3.13 in [23]) and the construction of $e^{t A}$ with respect to the Hilbert structures of $\mathrm{H}$ and $\mathrm{H}_{\mathbb{R}}$ are equal to each other we immediately get the thesis. Point (b). Again, the thesis hold for real Hilbert spaces and an operator over $\mathrm{H}$ is (anti) symmetric or (anti) self-adjoint over $\mathrm{H}$ if and only if it is so over $\mathrm{H}_{\mathbb{R}}$. The thesis follows immediately.

Theorem 4.10 The Nelson subspace $D_{N}^{(U)}$ satisfies the following properties.

(a) $D_{N}^{(U)} \subset D_{G}^{(U)}$

(b) $U_{g}\left(D_{N}^{(U)}\right) \subset D_{N}^{(U)}$ for any $g \in G$

(c) $D_{N}^{(U)}$ is dense in $\mathrm{H}$

(d) $D_{N}^{(U)}$ consists of analytic vectors for every operator $u(\mathbf{A})$ with $\mathbf{A} \in \mathfrak{g}$

(e) $u(\mathbf{A})\left(D_{N}^{(U)}\right) \subset D_{N}^{(U)}$ for any $\mathbf{A} \in \mathfrak{g}$.

(f) Let $p: \mathbb{R} \rightarrow \mathbb{R}$ be a real polynomial such that either

$$
p(-x)=p(x) \text { for every } x \in \mathbb{R} \text { or } p(-x)=-p(x) \text { for every } x \in \mathbb{R} .
$$

If $\mathbf{A} \in \mathfrak{g}$ then $\overline{u(p(\mathbf{A}))}$ is, respectively, selfadjoint or anti selfadjoint.

Proof. The thesis holds for real Hilbert spaces (Thm 3.14 in [23]). So, taking into account the equalities $D_{G}^{(U)}=D_{G}^{\left(U_{\mathbb{R}}\right)}$ and $D_{N}^{(U)}=D_{N}^{\left(U_{\mathbb{R}}\right)}$ and Remark 4.8 we immediately get the result.

\subsection{Group representations restricted to a complex Hilbert subspace induced by a complex structure}

To conclude this section we study the case of a group representation $U$ in a quaternionic Hilbert space equipped with a complex structure commuting with $U$. We start with the most elmentary situation. 
Lemma 4.11 Let $J$ be a complex structure in the quaternionic Hilbert space $\mathrm{H}$ and and $U: \mathbb{R} \ni t \mapsto U_{t} \in \mathfrak{B}(\mathrm{H})$ a strongly-continuous one-parameter group of unitary operators such that $J U_{t}=U_{t} J$ for every $t \in \mathbb{R}$. If $A$ is the anti-selfadjoint generator of $U$, then

(a) $A J=J A$

(b) $A_{J}$ is the anti-selfadjoint generator of $U_{J}: \mathbb{R} \ni t \mapsto\left(U_{t}\right)_{J} \in \mathfrak{B}\left(\mathrm{H}_{J}\right)$.

Proof. We know from Stone Theorem that $U_{t}=e^{t A}$ for some anti-selfadjoint operator $A: D(A) \rightarrow \mathrm{H}$. Suppose that $U_{t} J=J U_{t}$ for every $t \in \mathbb{R}$, then Lemma 2.15 yields $J A \subset A J$. Since $J$ is unitary and antiselfadjoint this immediately leads to $J A=A J$. $A_{J}$ is anti-selfadjoint and defined on $D\left(A_{J}\right)=D(A) \cap \mathrm{H}_{J}$ by Prop.3.4 and $x \in D\left(A_{J}\right)$ if and only if $x \in \mathrm{H}_{J}$ and there exists $\lim _{t \rightarrow 0} \frac{U_{t} x-x}{t}=\lim _{t \rightarrow 0} \frac{\left(U_{t}\right)_{J} x-x}{t}=A_{J} x$ due to Stone theorem. This is exactly the definition of the generator of $U_{J}$ proving the thesis.

We pass to the main result.

Proposition 4.12 Let $U: G \ni g \mapsto U_{g} \in \mathfrak{B}(\mathrm{H})$ a strongly-continuous unitary Lie-group representation over the quaternionic Hilbert space $\mathrm{H}$ and $J$ a complex structure such that $J U_{g}=U_{g} J$ for every $g \in G$. Then the following facts hold

(a) $J\left(D_{G}^{(U)}\right)=D_{G}^{(U)}$

(b) $U_{J}: G \ni g \mapsto\left(U_{g}\right)_{J} \in \mathfrak{B}\left(\mathrm{H}_{J}\right)$ is a strongly-continuous unitary representation over $\mathrm{H}_{J}$

(c) $D_{G}^{\left(U_{J}\right)}=D_{G}^{(U)} \cap \mathrm{H}_{J}$,

(d) $J u(\mathbf{M})=u(\mathbf{M}) J$ for all $\mathbf{M} \in E_{\mathfrak{g}}$. Moreover $u_{J}(\mathbf{M})=u(\mathbf{M})_{J}$.

Proof. See Appendix A

4.4 Group representations restricted to a real Hilbert subspace induced by two anti commuting complex structures

Lemma 4.13 Let $J, K$ be anticommuting complex structures in the quaternionic Hilbert space $\mathrm{H}$ and and $U: \mathbb{R} \ni t \mapsto U_{t} \in \mathfrak{B}(\mathrm{H})$ a strongly-continuous one-parameter group of unitary operators such that $J U_{t}=U_{t} J$ and $K U_{t}=U_{t} K$ for every $t \in \mathbb{R}$. If $A$ is the antiselfadjoint generator of $U$, then

(a) $A J=J A$ and $A K=K A$,

(b) $A_{J K}$ is the anti-selfadjoint generator of $U_{J K}: \mathbb{R} \ni t \mapsto\left(U_{t}\right)_{J K} \in \mathfrak{B}\left(\mathrm{H}_{J K}\right)$.

Proof. Analogous to the proof of Lemma 4.11 
Proposition 4.14 Let $U: G \ni g \mapsto U_{g} \in \mathfrak{B}(\mathrm{H})$ a strongly-continuous unitary Liegroup representation over the quaternionic Hilbert space $\mathrm{H}$ and Let $J, K$ be anticommuting complex structures such that $J U_{g}=U_{g} J$ and $K U_{g}=U_{g} K$ for every $g \in G$. Then the following facts hold

(a) $J\left(D_{G}^{(U)}\right) \subset D_{G}^{(U)}$ and $K\left(D_{G}^{(U)}\right) \subset D_{G}^{(U)}$

(b) $U_{J K}: G \ni g \mapsto\left(U_{g}\right)_{J K} \in \mathfrak{B}\left(\mathrm{H}_{J K}\right)$ is a strongly-continuous unitary representation over $\mathrm{H}_{J K}$

(c) $D_{G}^{\left(U_{J K}\right)}=D_{G}^{(U)} \cap \mathrm{H}_{J K}$

(d) $u(\mathbf{M}) J=J u(\mathbf{M})$ and $u(\mathbf{M}) K=K u(\mathbf{M})$ for all $\mathbf{M} \in E_{\mathfrak{g}}$. Moreover $u_{J K}(\mathbf{M})=$ $u(\mathbf{M})_{J K}$.

Proof. The proof is analogous to Proposition 4.12]s one.

\section{$5 \quad$ Irreducibility and Schur's Lemma on Quaternionic Hilbert spaces}

The notion of irreducibility will play crucial role in our work.

Definition 5.1 Let $\mathrm{H}$ be a real, complex or quaternionic Hilbert space. A family of operators $\mathfrak{S} \subset \mathfrak{B}(\mathrm{H})$ is said to be irreducible if $U(\mathrm{~K}) \subset \mathrm{K}$ for all $U \in \mathfrak{R}$ and a closed subspace $\mathrm{K} \subset \mathrm{H}$ implies $\mathrm{K}=\{0\}$ or $\mathrm{K}=\mathrm{H}$. $\mathfrak{S}$ is said to be reducible if it is not irreducible.

Since the definition refers to closed subspaces, our notion of irreducibility is sometimes called topological irreducibility.

\section{Remark 5.2}

(a) If $\mathfrak{S}$ is irreducible, then $\mathfrak{S}^{\prime} \cap \mathcal{L}(\mathrm{H})=\{0, I\}$. (This is because if an orthogonal projector $P$ commutes with all elements of $\mathfrak{S}$, then the closed subspace $\mathrm{K}:=P(\mathrm{H})$ is invariant under $\mathfrak{S})$.

(b) $\mathfrak{S}^{\prime} \cap \mathcal{L}(\mathrm{H})=\{0, I\}$ is equivalent to irreducibility of $\mathfrak{S}$ when $\mathfrak{S}$ is closed under Hermitian conjugation. (This is because $U(\mathrm{~K}) \subset \mathrm{K}$ implies $P U P=U P$ if $P$ is the orthogonal projector onto $\mathrm{K}$. If this holds for every $U \in \mathfrak{R}$ which is closed under Hermitian conjugation, $P U^{*} P=U^{*} P$ holds as well. Taking the Hermitian conjugate, $P U P=P U$, so that $U P=P U$.)

\subsection{Schur's lemma in quaternionic Hilbert spaces}

Let us pass to formulate the quaternionic version of Schur's Lemma which, as in the real Hilbert space case, has a formulation more complicated than in the standard complex Hilbert space case. 
Proposition 5.3 (Schur's lemma for essentially selfadjoint operators) Let $\mathrm{H}$ be a quaternionic Hilbert space and let $\mathfrak{S} \subset \mathfrak{B}(\mathrm{H})$ be irreducible.

If the operator $A: D(A) \rightarrow \mathrm{H}$, with $D(A) \subset \mathrm{H}$ dense, is essentially selfadjoint and

$$
U A \subset A U \text { for all } U \in \mathfrak{S}
$$

then $\bar{A} \in \mathfrak{B}(\mathrm{H})$ (the bar denoting the closure of $A$ ) and

$$
\bar{A}=a I, \quad \text { for some } a \in \mathbb{R} .
$$

If $A$ satisfying (25) is selfadjoint, then $A \in \mathfrak{B}(\mathrm{H})$ with $A=a I$ for some $a \in \mathbb{R}$.

Proof. The proof, based on the spectral theorem, is essentially identical to that of Prop. 2.13 in [23] which is valid for real and complex Hilbert spaces.

A different and more precise result can be obtained when the class $\mathfrak{R}$ consists of a *-closed subset of $\mathfrak{B}(\mathrm{H})$. In this case the statement is different from the one valid in complex Hilbert spaces but the same statement holds in real Hibert spaces.

Proposition 5.4 Let $\mathrm{H}$ be a quaternionic Hilbert space, $\mathfrak{S} \subset \mathfrak{B}(\mathrm{H}) a^{*}$-closed subset and consider a densely-defined closed operator in $\mathrm{H} A: D(A) \rightarrow \mathrm{H}$ such that

$$
U A=A U, \quad U A^{*}=A^{*} U, \quad \forall U \in \mathfrak{S} .
$$

If $\mathfrak{S}$ is irreducible then $A=a I+b J$ for some $a, b \in \mathbb{R}$ and $J$ a complex structure. In particular $D(A)=\mathrm{H}$ and $A \in \mathfrak{B}(\mathrm{H})$ in both cases.

Proof. $A U=U A$ and $A^{*} U=U A^{*}$ imply $A^{*} A U=A^{*} U A=U A^{*} A$ on natural domains. Since $A$ is closed, the operator $A^{*} A$ is densely defined and selfadjoint (Thm 2.14). Proposition 5.3 for the selfadjoint operator $A^{*} A$ implies $A^{*} A=a I$ for some real $a$. In particular $D\left(A^{*} A\right)=D(a I)=\mathrm{H}$ so that $D(A)=\mathrm{H}$ and thus, since $A$ is closed, the closed graph theorem ([11] Prop.2.11) gives $A \in \mathfrak{B}(\mathrm{H})$. To go on, decompose $A=\frac{A+A^{*}}{2}+\frac{A^{*}-A}{2}$ where the two addends denoted by $A_{S}$ and $A_{A}$ are, respectively, selfadjoint and anti selfadjoint belong to $\mathfrak{B}(\mathrm{H})$ and commute with the elements of $\mathfrak{S}$. In particular, $U A_{S}=A_{S} U$ for any $U \in \mathfrak{S}$ gives $A_{S}=a I$ for some $a \in \mathbb{R}$, thanks to Prop.5.3. Similarly, $A_{A}^{2} \in \mathfrak{B}(\mathrm{H})$ is selfadjoint and commutes with the operators in $\mathfrak{S}$, hence $A_{A}^{2}=c I$ for some $c \in \mathbb{R}$, thanks again to Proposition 5.3. It must be $c \leq 0$ because, if $v \in \mathrm{H}$ has unit norm, $c=\langle v \mid c v\rangle=\left\langle v \mid A_{A} A_{A} v\right\rangle=-\left\langle A_{A} v \mid A_{A} v\right\rangle=-\left\|A_{A} v\right\|^{2} \leq 0$. In particular, $c=0$ if and only if $A_{A}=0$, that is if $A$ is selfadjoint and in this case the proof ends. In the case $c \neq 0$, define $J:=\frac{A_{A}}{\sqrt{-c}}$. With this definition we find $J \in \mathfrak{B}(\mathrm{H}), J^{*}=-J$ and $J^{*} J=-I$ so that $J$ is a complex structure and $A=a I+b J$ for $a, b \in \mathbb{R}$ ending the proof again.

\section{Remark 5.5}

(a) If $\mathfrak{S}:=\left\{U_{g} \mid g \in G\right\}$ for a unitary group representation $G \ni g \mapsto U_{g} \in \mathfrak{B}(\mathrm{H})$, the hypothesis (26) of Proposition 5.4 can be weakened to

$$
U_{g} A \subset A U_{g}, \quad \forall g \in G .
$$


Indeed, multiplying both side by $U_{g^{-1}}$ on the left and by $U_{g}$ on the right, we get $A U_{g} \subset U_{g} A$ so that $U_{g} A=A U_{g}$. Taking the adjoint of this identity we also have $U_{g}^{*} A^{*} \subset A^{*} U_{g}^{*}$, because $U_{g}$ is bounded. Since $U_{g}^{*}=U_{g^{-1}}$ and $g$ varies on the whole set $G$ we have found $U_{g} A^{*} \subset A^{*} U_{g}$ and thus $U_{g} A^{*}=A^{*} U_{g}$ with the same reasoning as above, recovering (26). (b) If $A$ is bounded, the hypothesis (26) of Proposition 5.4 can be weakened to $U A=A U$ for every $U \in \mathfrak{S}$. Indeed the second identity in (26) immediately follows from $U A=A U$ and ${ }^{*}$-closure of $\mathfrak{S}$.

(c) In general, irreducibility of a unitary representation of a group on $\mathrm{H}$ is lost when moving from the quaternionic Hilbert space structure to the underlying real Hilbert space one $\mathrm{H}_{\mathbb{R}}$. Indeed consider the following example. Let $\mathrm{H}=\mathbb{H}$ and $G=S O(3)$ and define the representation $G \ni R \mapsto U_{R}$ defined by $U_{R}(a, \mathbf{b}):=(a, R \mathbf{b})$ for all $(a, \mathbf{b}) \in \mathbb{H}$. This is clearly unitary and irreducible (we are working on a one-dimensional Hilbert space). Of course $\mathrm{H}_{\mathbb{R}}=\mathbb{R}^{4}$ and $U_{R}(a, \mathbf{0})=(a, \mathbf{0})$ for all $a \in \mathbb{R}$, hence the one-dimensional subspace $\{(a, \mathbf{0}) \mid a \in \mathbb{R}\}$ is invariant under the action of $U$. This make the representation reducible on $\mathbb{R}^{4}$.

To conclude this general part let us consider the case of *-closed subset of $\mathfrak{B}(\mathrm{H})$ equipped with one or two complex structures.

Proposition 5.6 Let $\mathfrak{S} a^{*}$-closed subset of $\mathfrak{B}(\mathrm{H})$ for a quaternionic Hilbert space $\mathrm{H}$. The following facts hold.

(a) If there is a complex structure $J \in \mathfrak{S}^{\prime}$, then the set of complex-linear operators $\mathfrak{S}_{J}:=\left\{A_{J} \mid A \in \mathfrak{S}\right\} \subset \mathfrak{B}\left(\mathrm{H}_{J}\right)$ is irreducible if $\mathfrak{S}$ is.

(b) If there is a pair of complex structures $J, K \in \mathfrak{S}^{\prime}$ with $J K=-K J$, then the set of real-linear operators $\mathfrak{S}_{J K}:=\left\{A_{J K} \mid A \in \mathfrak{S}\right\} \subset \mathfrak{B}\left(\mathrm{H}_{J K}\right)$ is irreducible if $\mathfrak{S}$ is.

Proof. Dealing with ${ }^{*}$-closed sets, irreducibility is equivalent to the non-existence of nontrivial projectors commuting with the algebra for (b) Remark 5.2.

(a) Suppose that $\mathfrak{S}$ is irreducible and let $P \in \mathcal{L}\left(\mathrm{H}_{J}\right) \cap\left(\mathfrak{S}_{J}\right)^{\prime}$. We have $P=\left.\widetilde{P}\right|_{\mathrm{H}_{J}}$ for some $\widetilde{P} \in \mathfrak{B}(\mathrm{H})$, which is clearly an orthogonal projector on $\mathrm{H}$ for Prop 3.4 because $P$ is. That proposition also implies that $P$ commutes with every element of $\mathfrak{S}_{J}$ if and only if $\widetilde{P}$ commutes with every element of $\mathfrak{S}$. Since $\mathfrak{S}$ is irreducible we have the thesis. The proof of (b) is essentially identical.

\subsection{Application to Lie-group representations}

A remarkable consequence of the properties of Nelson's technology and our version of Schur's lemma for Lie group representations is the following proposition.

Proposition 5.7 Let $g \mapsto U_{g}$ be an irreducible strongly-continuous unitary representation of a connected Lie group over a quaternionic Hilbert space $\mathbf{H}$ and $\mathbf{M} \in E_{\mathfrak{g}}$ such that 
(a) $u(\mathbf{M})$ is essentially self-adjoint,

(b) $[\mathbf{M}, \mathbf{A}]_{\mathfrak{g}}=0 \quad \forall \mathbf{A} \in \mathfrak{g}$

then it holds $u(\mathbf{M})=\left.c I\right|_{D_{G}^{(U)}}$ for some $c \in \mathbb{R}$.

In particular the thesis holds if $\mathbf{M}=\mathbf{M}^{+}$and (b) is valid.

Proof. Let $x \in D_{N}^{(U)}$ and $\mathbf{A} \in \mathfrak{g}$. Thanks to Theorem 4.10 it holds $x \in D_{G}^{(U)}$ and $x$ is analytic for $u(\mathbf{A})$, in particular it is analytic for $\overline{u(\mathbf{A})}$. Exploiting Prop 4.9, we have that there exists $t_{\mathbf{A}, x}>0$ such that

$$
U_{\exp (t \mathbf{A})} x=e^{t \overline{u(\mathbf{A})}} x=\sum_{n=0}^{\infty} \frac{t^{n}}{n !} u(\mathbf{A})^{n} x, \quad|t| \leq t_{\mathbf{A}, x} .
$$

Moreover $D_{N}^{(U)}$ is invariant under the action of $u$, hence $u(\mathbf{M}) x \in D_{N}^{(U)}$. Then there exits $t_{\mathbf{A}, u(\mathbf{M}) x}>0$ such that

$$
U_{\exp (t \mathbf{A})} u(\mathbf{M}) x=e^{\overline{t u(\mathbf{A})}} u(\mathbf{M}) x=\sum_{n=0}^{\infty} \frac{t^{n}}{n !} u(\mathbf{A})^{n} u(\mathbf{M}) x, \quad|t| \leq t_{\mathbf{A}, u(\mathbf{M}) x} .
$$

Now take a positive real $t_{x}<\min \left\{t_{\mathbf{A}, x}, t_{\mathbf{A}, u(\mathbf{M}) x}\right\}$. Using $[u(\mathbf{M}), u(\mathbf{A})]=0$ we have

$$
U_{\exp (t \mathbf{A})} u(\mathbf{M}) x=\sum_{n=0}^{\infty} \frac{t^{n}}{n !} u(\mathbf{M}) u(\mathbf{A})^{n} x, \quad|t| \leq t_{x} .
$$

Since $u(\mathbf{M})$ is closable, it follows directly from the equations above and the invariance of $D_{G}^{(U)}$ under the action of $U$ that

$$
U_{\exp (t \mathbf{A})} u(\mathbf{M}) x=\sum_{n=0}^{\infty} \frac{t^{n}}{n !} u(\mathbf{M}) u(\mathbf{A})^{n} x=u(\mathbf{M}) U_{\exp (t \mathbf{A})} x
$$

for every $|t| \leq t_{x}$. Actually this equality holds for every $t \in \mathbb{R}$. Indeed define $\mathcal{Z}:=\{z>$ $\left.0\left|u(\mathbf{M}) U_{\exp (t \mathbf{A})} x=U_{\exp (t \mathbf{A})} u(\mathbf{M}) x,\right| t \mid \leq z\right\}$ and let $t_{0}:=\sup \mathcal{Z}$. Suppose that $t_{0}<\infty$, then it is easy to see that the fact that $u(\mathbf{M})$ is closable ensures that $u(\mathbf{M}) U_{\exp \left(t_{0} \mathbf{A}\right)} x=$ $U_{\exp \left(t_{0} \mathbf{A}\right)} u(\mathbf{M}) x$, hence $t_{0} \in \mathcal{Z}$. We know that $y:=U_{\exp \left(t_{0} \mathbf{A}\right)} x \in D_{N}^{(U)}$, we can therefore repeat the above reasoning finding a real $t_{y}>0$ such that $u(\mathbf{M}) U_{\exp (t \mathbf{A})} y=U_{\exp (t \mathbf{A})} u(\mathbf{M}) y$ for every $|t| \leq t_{y}$. Noticing that $\exp \left(\left(t+t_{0}\right) \mathbf{A}\right)=\exp (t \mathbf{A}) \exp \left(t_{0} \mathbf{A}\right)$, it straightforwardly follows that $u(\mathbf{M}) U_{\exp \left(t+t_{0}\right) \mathbf{A}} x=U_{\exp \left(t+t_{0}\right) \mathbf{A}} u(\mathbf{M}) x$ for $|t| \leq t_{y}$, hence $t_{0}+t_{y} \in \mathcal{Z}$, which is in contradiction with the definition of $t_{0}$. This proves that $t_{0}=\infty$. As is well known from the elementary theory of Lie-group theory, since the $G$ is connected, every element is the product of a finite number of elements belonging to one parameter subgroups generated by $\mathfrak{g}$, so that we have actually demonstrated that $u(\mathbf{M}) U_{g}=U_{g} u(\mathbf{M})$ on $D_{N}^{(U)}$ 
for every $g \in G$. This identity implies $\left.U_{g} u(\mathbf{M})\right|_{D_{N}^{(U)}}=\left.u(\mathbf{M})\right|_{D_{N}^{(U)}} U_{g}$ on the natural domains thanks to the invariance of the Nelson space under the action of the group representation. In our hypotheses, $\left.u(\mathbf{M})\right|_{D_{N}^{(U)}}$ is the restriction of a closable operators and thus it is closable as well and so $U_{g} \overline{\left.u(\mathbf{M})\right|_{D_{N}^{(U)}}}=\overline{\left.u(\mathbf{M})\right|_{D_{N}^{(U)}}} U_{g}$ for every $g$. Using Proposition 5.4 and Remark 5.5 (a) we find $D\left(\overline{\left.u(\mathbf{M})\right|_{D_{N}^{(U)}}}\right)=\mathrm{H}$ and $\overline{\left.u(\mathbf{M})\right|_{D_{N}^{(U)}}} \in \mathfrak{B}(\mathbf{H})$, more precisely $\overline{\left.u(\mathbf{M})\right|_{D_{N}^{(U)}}}=a I+b J$ for some $a, b \in \mathbb{R}$, where $J$ is some complex structure. Since $\overline{\left.u(\mathbf{M})\right|_{D_{N}^{(U)}}} \subset \overline{u(\mathbf{M})}$, the maximality of the domain gives $\overline{\left.u(\mathbf{M})\right|_{D_{N}^{(U)}}}=\overline{u(\mathbf{M})}$. As the latter is selfadjoint, it follows that $b=0$ and $\overline{u(\mathbf{M})}=a I$ with $a \in \mathbb{R}$ ending the proof of the first statement. If $\mathbf{M}=\mathbf{M}^{+}$then $u(\mathbf{M})$ is symmetric for Thm 4.5 and, for the same theorem, it is also essentially self-adjoint because (b) implies that $\mathbf{M}$ commutes with a Nelson element.

\section{$6 \quad$ Irreducible quaternionic von Neumann algebras}

This section is devoted to focus on the basic properties of irreducible quaternionic von Neumann algebras.

\subsection{The commutant of an irreducible quaternionic von Neumann algebra}

An irreducible ${ }^{*}$-closed subset $\mathfrak{S} \subset \mathfrak{B}(\mathrm{H})$ in a complex Hilbert space is trivial in view of the complex version of Schur lemma. For real and quaternionic Hilbert spaces the picture is more complicated. In principle, the commutant of a generic ${ }^{*}$-closed irreducible subset $\mathfrak{S} \subset \mathfrak{B}(\mathrm{H})$ may contain infinitely different complex structures as suggested by Proposition 5.4. We now examine the quaternionic Hilbert space case when $\mathfrak{S}$ is a von Neumann algebra, finding a result similar Thm 5.3 established in [23] dealing with von Neumann algebras in real Hilbert spaces.

Theorem 6.1 Let $\mathfrak{R}$ be a von Neumann algebra on the quaternionic Hilbert space $\mathrm{H}$. If $\mathfrak{R}$ is irreducible, then $\mathfrak{R}^{\prime}$ is of three possible mutually exclusive types:

- $\mathfrak{R}^{\prime}=\{a I \mid a \in \mathbb{R}\}$ (quaternionic-real type).

- $\mathfrak{R}^{\prime}=\{a I+b J \mid a, b \in \mathbb{R}\}$ where $J$ is a complex structure determined up to its sign. Furthermore $J \in \mathfrak{R}$ (quaternionic-complex type).

- $\mathfrak{R}^{\prime}=\{a I+b J K+c J+d K \mid a, b, c, d \in \mathbb{R}\}$ where $J, K$ and $J K=-K J$ are complex structures. Furthermore $J, K, J K \notin \mathfrak{R}$ (quaternionic-quaternionic type)

In both the quaternionic-real and quaternionic-quaternionic cases the centre $\mathfrak{Z}_{\mathfrak{R}}$ of $\mathfrak{R}$ is $\mathfrak{Z}_{\mathfrak{R}}=\{a I \mid a \in \mathbb{R}\}$, while in the quaternionic-complex case $\mathfrak{Z}_{\mathfrak{R}}=\{a I+b J \mid a, b \in \mathbb{R}\}$

Proof. If $A \in \mathfrak{R}^{\prime}$, Prop. 5.4 implies $A=a I+b L$ for some $a, b \in \mathbb{R}$ and some complex structure $L$. As a consequence, $\mathfrak{R}^{\prime}$ is a real associative unital normed algebra with the 
further property that $\|A B\|=\|A\|\|B\|$. (Indeed, by direct computation $\|(a I+b L) x\|^{2}=$ $\left(a^{2}+b^{2}\right)\|x\|^{2}$ so that $\|a I+b L\|^{2}=a^{2}+b^{2}$. Furthermore, iterating the procedure, where $L^{\prime}$ is another complex structure, $\left\|(a I+b L)\left(a^{\prime} I+b^{\prime} L^{\prime}\right) x\right\|^{2}=\left(a^{2}+b^{2}\right)\left(a^{\prime 2}+b^{\prime 2}\right)\|x\|^{2}=$ $\left.\|a I+b L\|^{2}\left\|a^{\prime} I+b^{\prime} L^{\prime}\right\|\right|^{2}\|x\|^{2}$ and thus $\left.\left\|(a I+b L)\left(a^{\prime} I+b^{\prime} L^{\prime}\right)\right\|=\|a I+b L\|\left\|a^{\prime} I+b^{\prime} L^{\prime}\right\|.\right)$ Due to [38] there exists a real associative unital normed algebra isomorphism $h$ from $\mathfrak{R}^{\prime}$ to $\mathbb{R}, \mathbb{C}$ or $\mathbb{H}$. In the first case, $\mathfrak{R}^{\prime}=h^{-1}(\mathbb{R})=\{a I \mid a \in \mathbb{R}\}$. In the second case, $\mathfrak{R}^{\prime}=h^{-1}(\mathbb{C})=\{a I+b J \mid a, b \in \mathbb{R}\}$ where $J:=h^{-1}(i)$. Furthermore, as $h^{-1}$ is an isomorphism, $J J=h^{-1}(j j)=h^{-1}(-1)=-I$. In the third case, $\mathfrak{R}^{\prime}=h^{-1}(\mathbb{H})=$ $\{a I+b J K+c J+d K \mid a, b, c, d \in \mathbb{R}\}$ with $J:=h^{-1}(j), K:=h^{-1}(k), J K:=h^{-1}(i)$ where $i, j, k \in \mathbb{H}$ (with $i=j k=-k j$ ) are the three imaginary units. Again, as in the realcomplex case, we get $J J=h^{-1}(j j)=h^{-1}(-1)=-I$ and $K K=h^{-1}(k k)=h^{-1}(-1)=$ $-I$. Let us prove that $J$ in the quaternionic-complex case and $J, K$ in the quaternionicquaternionic one are antiselfadjoint concluding that they are complex structures. The proof being the same in both cases, we deal with $J$ only. Since $\mathfrak{R}^{\prime}$ is a ${ }^{*}$-algebra, it holds $J^{*} \in \mathfrak{R}^{\prime}$, in particular $J^{*} J \in \mathfrak{R}^{\prime}$ which is clearly self-adjoint and positive. Since $\mathfrak{R}$ is irreducible, Proposition 5.3 guarantees that $J^{*} J=a I$ for some $a \geq 0$. Multiplying both sides by $-J$ on the right, using $J J=-I$, we get $J^{*}=-a J$. Taking the adjoint on both sides yields $J=-a J^{*}$ which, in particular, assures that $a \neq 0, J$ being bijective since $J J=-I$. So, $J^{*}=-\frac{1}{a} J$. Summing up, $0=J^{*}-J^{*}=\left(a-\frac{1}{a}\right) J$. As $J J=-I$, it must be $a-\frac{1}{a}=0$, hence $a=1$ and $J^{*}=-a J=-J$ as wanted. $J$ is a complex structure. $J K$ turns out to be a complex structure as well, since $J$ and $K$ are complex structures and $J K=-K J$.

To conclude, let us establish the form of the centers $\mathfrak{Z}_{\Re}$. The real case is obvious. In the complex case, $J$ commutes with $\{a I+b J \mid a, b \in \mathbb{R}\}=\mathfrak{R}^{\prime}$, so it belongs to $\mathfrak{R}^{\prime \prime}=\mathfrak{R}$ and thus $\mathfrak{Z}_{\mathfrak{R}}=\{a I+b J \mid a, b \in \mathbb{R}\}$. This result also implies that, in the complex case, $J$ is unique up to its sign. Indeed, let $J^{\prime}$ be another complex structure in $\mathfrak{R}^{\prime}$, then it commutes with $J$ (as it belongs to $\mathfrak{R}$ ). Therefore $J J^{\prime} \in \mathfrak{R}^{\prime}$ is self adjoint and thus $J J^{\prime}=a I$, namely $J^{\prime}=-a J$, because $\mathfrak{R}$ is irreducible. Since $J J=J^{\prime} J^{\prime}=-1$ we must have $a= \pm 1$. $\mathfrak{Z}_{\mathfrak{R}}$ for the quaternionic case is easy. Suppose that $U=a+b J+c K+$ $d J K \in \mathfrak{R}$ for some $a, b, c, d \in \mathbb{R}$, then, since $\mathfrak{R}=\mathfrak{R}^{\prime \prime}$ it must be $U J=J U$, that is $a J+b J^{2}+c K J+d J K J=a J+b J^{2}+c J K+d J^{2} K$. A straightforward calculcation shows that $2 c J-2 d=0$ which, taking the Hermitean conjugate, is equivalent to $2 d+2 c J=0$. Combining the two equations we get $d=c=0$. Finally, since it must also be $U K=K U$ we get $a K+b J K=a K+b K J$ which simplifies as $b J K=b K J$. Since $J, K$ anticommute with each other it must be $b=0$. This concludes the proof.

\subsection{The structure of irreducible von Neumann algebras in quaternionc Hilbert spaces.}

We are in a position to prove that the structure of irreducible von Neumann algebras and associated lattice of orthogonal projectors is isomorphic the one of $\mathfrak{B}\left(\mathrm{H}^{\prime}\right)$ where $\mathrm{H}^{\prime}$ is suitable. This result will play a central and crucial role in the rest of this work.

Theorem 6.2 Let $\mathfrak{R}$ be an irreducible von Neumann algebra in the quaternionic Hilbert 
space $\mathrm{H}$. Referring to the three cases listed in Theorem 6.1, the following facts hold.

(a) If $\mathfrak{R}^{\prime}=\{a I \mid a \in \mathbb{R}\}$, then

(i) $\mathfrak{R}=\mathfrak{B}(\mathrm{H})$,

(ii) $\mathcal{L}_{\mathfrak{R}}(\mathrm{H})=\mathcal{L}(\mathrm{H})$.

(b) If $\mathfrak{R}^{\prime}=\{a I+b J \mid a, b \in \mathbb{R}\}$, then

(i) $\mathfrak{R}_{J}=\mathfrak{B}\left(\mathrm{H}_{J}\right)$ and the map $\mathfrak{R} \ni A \mapsto A_{J} \in \mathfrak{B}\left(\mathrm{H}_{J}\right)$ is a norm-preserving weakly-continuous (thus strongly-continuous) ${ }^{*}$-isomorphism of real unital ${ }^{*}$-algebras which, in particular, maps $J$ to $j I$.

(ii) the map $\mathcal{L}_{\mathfrak{R}}(\mathrm{H}) \ni P \mapsto P_{J} \in \mathcal{L}\left(\mathrm{H}_{J}\right)$ is an isomorphism of complete orthocomplemented lattices.

(c) If $\mathfrak{R}^{\prime}=\{a I+b J K+c J+d K \mid a, b, c, d \in \mathbb{R}\}$, then

(i) $\mathfrak{R}_{J K}=\mathfrak{B}\left(\mathrm{H}_{J K}\right)$ and the map $\mathfrak{R} \ni A \mapsto A_{J K} \in \mathfrak{B}\left(\mathrm{H}_{J K}\right)$ is a norm-preserving weakly-continuous (thus strongly-continuous) ${ }^{*}$-isomorphism of real unital ${ }^{*}$-algebras,

(ii) the map $\mathcal{L}_{\mathfrak{R}}(\mathrm{H}) \ni P \mapsto P_{J K} \in \mathcal{L}\left(\mathrm{H}_{J K}\right)$ is an isomorphism of complete orthocomplemented lattices.

Proof. (a) From $\mathfrak{R}^{\prime}=\{a I \mid a \in \mathbb{R}\}$ it immediately follows $\mathfrak{R}=\mathfrak{R}^{\prime \prime}=\mathfrak{B}(\mathrm{H})$ and thus $\mathcal{L}_{\mathfrak{R}}(\mathrm{H})=\mathcal{L}(\mathrm{H})$.

(b) (i) We know from Prop 3.6 that $\mathfrak{R}$ gives rise to a complex von Neumann algebra $\mathfrak{R}_{J}$, the latter von Neumann algebra being irreducible due to Prop. 5.6] since the former is irreducible. The complex version of Schur's lemma implies that $\mathfrak{R}_{J}=\mathfrak{B}\left(\mathrm{H}_{J}\right)$. The map $\mathfrak{R} \ni A \mapsto A_{J} \in \mathfrak{R}_{J}$ is a norm-preserving weakly-continuous, strongly-continuous ${ }^{*}$-isomorphism of real unital *-algebras in view of Prop. 3.4. Let us pass to (ii). Prop. 3.4 easly implies that $\mathcal{L}_{\mathfrak{R}}(\mathrm{H}) \ni P \mapsto P_{J} \in \mathcal{L}\left(\mathrm{H}_{J}\right)$ is an isomorphism of orthocomplemented lattices. The only pair of properties to be proved concerns completeness of the involved lattices of orthogonal projectors and are the following ones. (1) Given a family $\left\{P_{a}\right\}_{a \in A} \subset$ $\mathcal{L}_{\mathfrak{R}}(\mathrm{H})$ such that $\mathrm{M}_{a}:=P_{a}(\mathrm{H})$, defining $P=\inf _{a \in A} P_{a}$ - in other words $P$ is the orthogonal projector onto $\mathrm{M}:=\cap_{a \in A} \mathrm{M}_{a}$ - it turns out that $P_{J}=\inf _{a \in A}\left(P_{a}\right)_{J}$. Regarding the fact that $P_{J}$ is well defined, observe that $P \in \mathcal{L}_{\mathfrak{R}}(\mathrm{H})$ because $\mathcal{L}_{\mathfrak{R}}(\mathrm{H})$ is complete and thus $P$ commutes with $J$ since $\mathcal{L}_{\mathfrak{R}}(\mathrm{H}) \subset \mathfrak{R}$. Similarly, (2) Given a family $\left\{P_{a}\right\}_{a \in A} \subset \mathcal{L}_{\mathfrak{R}}(\mathrm{H})$ such that $M_{a}:=P_{a}(\mathrm{H})$ defining $Q=\sup _{a \in A} P_{a}$ - in other words $Q \in \mathfrak{R}$ is the orthogonal projector onto $\mathrm{N}:=\overline{\left\langle\cup_{a \in A} \mathrm{M}_{a}\right\rangle}$ - it turns out that $Q_{J}=\sup _{a \in A}\left(P_{a}\right)_{J}$. Regarding the fact that $Q_{J}$ is well defined, observe that $Q \in \mathcal{L}_{\mathfrak{R}}(\mathrm{H})$ because $\mathcal{L}_{\mathfrak{R}}(\mathrm{H})$ is complete and thus $Q$ commutes with $J$ since $\mathcal{L}_{\mathfrak{R}}(\mathrm{H}) \subset \mathfrak{R}$. To prove (1), observe that $P_{J}$ is the orthogonal projector onto $\mathrm{M} \cap \mathrm{H}_{J}$ because $x=P_{J} x$ if and only if both $x \in \mathrm{H}_{J}$ (because $P_{J}$ is a projector in $\left.\mathfrak{B}\left(\mathrm{H}_{J}\right)\right)$ and $x=P x$ which means $x \in \mathrm{M}$. We conclude that $P_{J}$ 
is the orthogonal projector onto $\mathrm{M} \cap \mathrm{H}_{J}=\left(\cap_{a \in A} \mathrm{M}_{a}\right) \cap \mathrm{H}_{J}=\cap_{a \in A}\left(\mathrm{M}_{a} \cap \mathrm{H}_{J}\right)$. The orthogonal projector onto the last space is $\inf _{a \in A}\left(P_{a}\right)_{J}$ by definition. We have obtained $P_{J}=\inf _{a \in A}\left(P_{a}\right)_{J}$. Property (2) is an immediate consequence of (1) and De Morgan's rule, valid for any family orthogonal projectors $\left\{Q_{b}\right\}_{b \in B} \subset \mathfrak{B}(\mathrm{K})$ with $\mathrm{K}$ real, complex or quaternionic, $\sup _{b \in B} Q_{b}=\left(\inf _{b \in B} Q_{b}^{\perp}\right)^{\perp}$, where $Q^{\perp}:=I-Q$ is the orthogonal projector onto $Q(\mathrm{~K})^{\perp}$.

(c) (i) Making use of Prop 3.9 and Propositions 3.11, 5.6 we have that $\mathfrak{R} \ni A \mapsto A_{J K} \in$ $\mathfrak{R}_{J K}$ is a norm-preserving weakly-continuous, strongly-continuous *-isomorphism of real unital *-algebras where $\mathfrak{R}_{J K}$ is an irreducible von Neumann algebra. Let us prove that $\mathfrak{R}_{J K}=\mathfrak{B}\left(\mathrm{H}_{J K}\right)$. Let $A \in \mathfrak{B}\left(\mathrm{H}_{J K}\right)$, then Prop 3.9 assures that there exists a unique $\widetilde{A} \in \mathfrak{B}(\mathrm{H})$ such that $A=\widetilde{A}_{J K}$. Moreover $\widetilde{A}$ commutes with $J$ and $K$. Since $\mathfrak{R}^{\prime}=$ $\{a I+b J+c K+d J K\}$, it immediately follows that $\widetilde{A} \in \mathfrak{R}^{\prime \prime}=\mathfrak{R}$, i.e., $A \in \mathfrak{R}_{J K}$. This means $\mathfrak{R}_{J K} \supset \mathfrak{B}\left(\mathrm{H}_{J K}\right)$ and thus $\mathfrak{R}_{J K}=\mathfrak{B}\left(\mathrm{H}_{J K}\right)$ because the converse inclusion is obvious. The proof of (ii) is essentially identical to the corresponding for (b), just noticing that now we have to handle two (anticommuting) complex structures $J$ and $K$.

\section{Quaternionic Wigner relativistic elementary systems (WRES)}

Within this section, as already done in [23], we introduce a first notion of elementary system with respect to the relativistic symmetry adopting the famous framework introduced by Wigner. A relativistic elementary system in Wigner's view is a quantum system completely determined from its symmetry properties where the symmetry group is Poincaré one. So it is determined by a faithful (with the caveat discussed below) continuous unitary representation of that group and that representation also fixes the class of observables of the system which coincide with the selfadjoint elements of the von Neumann algebra generated by the representation itself. Elementariness of the said system is translated into the irreduciblility demand of the representation. Later we will come back on these requirements improving this model as already done in [23].

\subsection{Faithfulness issues}

Actually by Poincaré group we will indicate here the real simply-connected Lie group obtained as the semi-direct product $S L(2, \mathbb{C}) \ltimes \mathbb{R}^{4}$ with respect to the abelian normal subgroup $\mathbb{R}^{4}$.

Definition 7.1 $\mathcal{P}$, called Poincaré group in this paper, is the semi-direct product $S L(2, \mathbb{C}) \ltimes \mathbb{R}^{4}$ of the groups $S L(2, \mathbb{C})$ and $\mathbb{R}^{4}$, with group product defined as

$$
(A, t) \cdot\left(A^{\prime}, t^{\prime}\right):=\left(A A^{\prime}, t+\Lambda(A) t^{\prime}\right) \quad \text { for } A, A^{\prime} \in S L(2, \mathbb{C}) \text { and } t, t^{\prime} \in \mathbb{R}^{4},
$$

where $\Lambda: S L(2, \mathbb{C}) \rightarrow S O(1,3) \uparrow$ is the standard covering homomorphism.

$\mathcal{P}$ is more properly known as the universal covering of the proper orthochronous Poincaré group as understood in relativity. $\mathcal{P}$ actually enters all known physical constructions and 
every representation of the proper orthochronous Poincaré group is also a representation of $\mathcal{P}$. For this reason, we require a weaker local-faithfulness assumption for every group representation of $\mathcal{P}$ we henceforth consider, i.e., the representation is only supposed to be injective in a neighbourhood of the neutral element of the group, because only in a neighbourhood of the identity of the group elements $S L(2, \mathbb{C})$ and the proper orthochronous Lorentz groups $S O(1,3) \uparrow$ are identical.

To corroborate our assumption, we remark that the complex strongly-continuous unitary irreducible representations of $S L(2, \mathbb{C}) \ltimes \mathbb{R}^{4}$ with physical meaning are all locally faithful: (1) for positive squared mass with semi-integer spin they are faithful, (2) for positive squared mass with integer spin they are faithful up to the sign of the $S L(2, \mathbb{C})$ element, and (3) they are faithful up to the sign of the $S L(2, \mathbb{C})$ element for zero squared mass with non-trivial momentum representation.

From a general point of view, local faithfulness of a continuous representation $\mathcal{P} \ni g \mapsto U_{g}$ is equivalent to the physically more natural requirement that

the representation of the subgroup $\mathbb{R}^{4}$ of spacetime translations is non-trivial.

More precisely, the following general result holds whose proof stays in Appendix B.

Proposition 7.2 Let $f: S L(2, \mathbb{C}) \ltimes \mathbb{R}^{4} \rightarrow G$ be a continuous group homomorphism to the topological Hausdorff group $G$. The following two facts are equivalent.

(a) $f$ is injective in a neighborhood of the unit element of $S L(2, \mathbb{C}) \ltimes \mathbb{R}^{4}$.

(b) The associated group homomorphism from the subgroup $\mathbb{R}^{4} \subset S L(2, \mathbb{C}) \ltimes \mathbb{R}^{4}$ to $G$

$$
\mathbb{R}^{4} \ni t \mapsto f((I, t))
$$

is not trivial.

If (a) and (b) are true, then either $f$ is injective or its kernel is $\{( \pm I, 0)\}$.

Due to Proposition 7.2, when $G$ is the group of unitary operators in the (real, complex or quaternionic) Hilbert space $\mathrm{H}$ equipped with the (Hausdorff) strong operator topology, a unitary strongly-continuous representation $\mathcal{P} \ni g \mapsto U_{g} \in G$ is locally faithful if and only if the associated representation of the group of spacetime translations is non-trivial. If $U$ is not faithfull the failure of injectivity is due to the sign of the elements in $S L(2, \mathbb{C})$ only.

\subsection{Wigner's approach extend to the quaternionic case}

Definition 7.3 A real, complex, quaternionic Wigner elementary relativistic system (WRES) is a unitary strongly continuous representation of the proper orthochronous Poincaré group $\mathcal{P}$,

$$
U: \mathcal{P} \ni g \mapsto U_{g} \in \mathfrak{B}(\mathrm{H})
$$

over the, respectively, real, complex, or quaternionic separable Hilbert space $\mathbf{H}$, and the representation is locally faithful (i.e., injective in a neighborhood of the unit element) and irreducible.

If $\mathfrak{R}_{U}$ is the von Neumann algebra generated by $U$, the observables of the WRES are 
the selfadjoint operators $A$ affiliated to $\Re_{U}$, i.e., their PVMs belong to $\mathcal{L}_{\mathfrak{R}_{U}}(\mathrm{H})$. $\mathcal{L}_{\mathfrak{R}_{U}}(\mathrm{H})$ itself is the set of elementary (YES-NO) observables of the WRES.

Remark 7.4 Every essentially self-adjoint operator of the form $u(\mathbf{M})$ for some $\mathbf{M} \in E_{\mathfrak{g}}$ defines an observable $\overline{u(\mathbf{M})}$ of the WRES. Indeed, every $u(\mathbf{M})$ satisfies $B u(\mathbf{M}) \subset u(\mathbf{M}) B$ and thus $B \overline{u(\mathbf{M})} \subset \overline{u(\mathbf{M})} B$ for every $B \in\left\{U_{g}\right\}_{g \in \mathcal{P}}^{\prime}=\Re_{U}^{\prime}$ as the reader can easily prove from Theorem 4.5, so that the PVM of the selfadjoint operator $\overline{u(\mathbf{M})}$ commutes with $B$ due to Lemma 2.11 and therefore it belongs to $\mathfrak{R}_{U}^{\prime \prime}=\mathfrak{R}_{U}$ as required for observables. We will generalize this example in Corollary 7.11 .

As established in the previous section for the quaternionic case and in [23] for the real and complex case, the representation $U$ gives rise to a representation on its Gårding domain of the corresponding Lie algebra $\mathfrak{g}$

$$
u: \mathfrak{g} \ni \mathbf{A} \mapsto u(\mathbf{A}) .
$$

Let us fix a Minkowskian reference frame in Minkowski spacetime. Referring to that reference frame, for $i=1,2,3, \mathbf{k}_{i} \in \mathfrak{g}$ denote the three generators of the boost oneparameter subgroups along the three spatial axes, $\mathbf{l}_{i} \in \mathfrak{g}$ are the three generators of the spatial rotations one-parameter subgroups about the three axes. Finally, for $\mu=0,1,2,3$ let $\mathbf{p}_{\mu} \in \mathfrak{g}$ be the four generators of the spacetime displacements one-parameter subgroups along the four Minkowskian axes. Next define the associated anti-selfadjoint generators

$$
\widetilde{K}_{i}:=\overline{u\left(\mathbf{k}_{i}\right)}, \widetilde{L}_{i}:=\overline{u\left(\mathbf{l}_{i}\right)}, \widetilde{P}_{0}:=\overline{u\left(\mathbf{p}_{0}\right)}, \widetilde{P}_{i}:=\overline{u\left(\mathbf{p}_{i}\right)} \quad i=1,2,3 .
$$

All of these operators leave fixed the Gåding domain $D_{G}^{(U)}$ which is a common core for all them. If we change the initially fixed reference frame by means of a transformation $p \in \mathcal{P}$, we obtain another set of generators related to the previous ones by the natural relations

$$
\widetilde{K}_{i}^{\prime}=U_{p} \widetilde{K}_{i} U_{p}^{-1}, \widetilde{L}_{i}^{\prime}=U_{p} \widetilde{L}_{i} U_{p}^{-1}, \widetilde{P}_{0}^{\prime}=U_{p} \widetilde{P}_{0} U_{p}^{-1}, \widetilde{P}_{i}^{\prime}=U_{p} \widetilde{P}_{i} U_{p}^{-1} i=1,2,3 .
$$

The said generators are not observables, since they are not selfadjoint and there is no trivial way to associate them with selfadjoint operators as instead it happens within the complex Hilbert space formulation where one has a standard imaginary unit at disposal. In turn, this problem makes it difficult to state a quantum version of Noether correspondence between generators of continuous symmetries and dynamically conserved quantities. For the moment we simply ignore these open issues.

Consider the element $\mathbf{e}:=-\mathbf{p}_{0}^{2}+\sum_{i=1}^{3} \mathbf{p}_{i}^{2} \in E_{\mathfrak{g}}$ and define the Mass operator as the symmetric operator

$$
M_{U}^{2}:=u(\mathbf{e})=\left.\left(-\widetilde{P}_{0}^{2}+\sum_{i=1}^{3} \widetilde{P}_{i}^{2}\right)\right|_{D_{G}^{(U)}} .
$$

By direct inspection one sees that $\mathbf{e}$ is symmetrice and commutes with all generators and thus, for Proposition [5.7, $M_{U}^{2}$ is essentially selfadjoint - thus represents an observable 
of the WRES in view of Remark 7.4 - and has a trivial form $c I$ for some constant $c \in \mathbb{R}$. Moreover it holds $U_{p} M_{U}^{2} U_{p}^{-1}=M_{U}^{2}$ for every $p \in \mathcal{P}$ and, in this sense, $M_{U}^{2}$ is Poincaré invariant. Indeed, exploiting the definition of Poincaré group and Stone Theorem, it is easy to see that $\left.U_{g} \tilde{P}_{\mu} U_{g}^{-1}\right|_{D_{G}^{(U)}}=\left.\sum_{\nu=0}^{3} \Lambda_{\mu}^{\nu} \tilde{P}_{\nu}\right|_{D_{G}^{(U)}}$ where $\Lambda$ is the Lorentz matrix corresponding to the element $g \in \mathcal{P}$ and this immediately yields the claim.

The real and complex cases were treated in [23], we now focus attention on the remaining quaternionic case taking advantage of the results already achieved in [23]. A first technical result is the following one.

Proposition 7.5 Let $U: \mathcal{P} \ni g \mapsto U_{g} \in \mathfrak{B}(\mathrm{H})$ be a WRES over the quaternionic Hilbert space $\mathrm{H}$. If $\widetilde{P}_{0}=J_{0}\left|\widetilde{P}_{0}\right|$ is the polar decomposition of the time displacement generator and $M_{U}^{2} \geq 0$, then $J_{0}$ is a complex structure in $\mathrm{H}$ with $J_{0} \in \mathfrak{R}_{U} \cap \mathfrak{R}_{U}^{\prime}$.

Proof. We need some preliminary results stated into some lemmata.

Lemma 7.6 With the hypotheses of Proposition 7.5, U defines reducible (stronglycontinuous locally-faithful) unitary representations both over the real Hilbert space $\mathbf{H}_{\mathbb{R}}$ and the complex Hilbert space $\mathrm{H}_{\mathbb{C}_{j}}$ as defined in Prop 2.1 and Prop 2.9 respectively.

Proof. Suppose $U: \mathcal{P} \ni g \mapsto U_{g}$ defines a WRES on a quaternionic Hilbert space $\mathrm{H}$. It is clear that $U$ is a strongly-continuous locally-faithful unitary representation over $\mathrm{H}_{\mathbb{R}}$ and $\mathrm{H}_{\mathbb{C}_{j}}$ as defined in Prop 2.1 and Prop 2.9 respectively and defines two corresponding representations called $U_{\mathbb{R}}$ and $U_{\mathbb{C}_{j}}$. Let us prove that the representations $U_{\mathbb{C}_{j}}$ on $\mathrm{H}_{\mathbb{C}_{j}}$ cannot be irreducible. First, remember that $D_{G}^{(U)}=D_{G}^{\left(U_{\mathbb{R}}\right)}=D_{G}^{\left(U_{\mathbb{C}_{j}}\right)}$ (Prop. 4.3) and the anti-selfadjoint generators of the one-parameter subgroups of spacetime displacements defined with respect to $\mathrm{H}$ or $\mathrm{H}_{\mathbb{R}}$ or $\mathrm{H}_{\mathbb{C}_{j}}$ are equal to each other (Prop. 4.4). Hence, $M_{U}^{2}=M_{U_{\mathbb{R}}}^{2}=M_{U_{\mathrm{c}_{j}}}^{2}$ and, it being symmetric, $M_{U}^{2}$ is positive on $\mathrm{H}$ if and only if it is positive on $\mathrm{H}_{\mathbb{R}}$ and, in turn, it happens if and only if it is positive on $\mathrm{H}_{\mathbb{C}_{j}}$. Thanks to Theorem 2.14, the polar decompositions $J_{0}\left|\widetilde{P}_{0}\right|$ of $\widetilde{P}_{0}$ defined with respect to the three considered spaces are identical. If the representation $U_{\mathbb{C}_{j}}$ were irreducible, due to $(\mathrm{g})$ in Theorem 4.3 in [23], we would have $J_{0}= \pm \mathcal{J}, \mathcal{J}$ being the imaginary unit of $\mathrm{H}_{\mathbb{C}_{j}}$. This is impossible, since $J_{0}$ is quaternionic linear and so it commutes with $\mathcal{K}$ if understood as a real operator on $\mathrm{H}_{\mathbb{R}}$, while we know that $\mathcal{J K}=-\mathcal{K} \mathcal{J}$. This also implies that $U_{\mathbb{R}}$ is reducible: If it were not the case, then $U_{\mathbb{C}_{j}}$ would be irreducible since $\mathrm{H}_{\mathbb{C}_{j}}=\left(\mathrm{H}_{\mathbb{R}}\right)_{\mathcal{J}}$ where the right-hand side is the internal complexification of $\mathrm{H}_{\mathbb{R}}$ referred to the complex structure $\mathcal{J}$ as in Sect.2.5 in [23].

Lemma 7.7 With the hypotheses of Proposition 7.5, there exists a projector $P \in \mathcal{L}\left(\mathrm{H}_{\mathbb{C}_{j}}\right)$ such that the orthogonal decomposition

$$
\mathrm{H}_{\mathbb{C}_{j}}=\mathrm{H}_{P} \oplus \mathrm{H}_{P^{\perp}}
$$

together with the following statements. 
(i) $\mathcal{J}\left(\mathrm{H}_{P}\right)=\mathrm{H}_{P}$ and $\mathcal{J}\left(\mathrm{H}_{P^{\perp}}\right)=\mathrm{H}_{P^{\perp}}$ while $\mathcal{K}\left(\mathrm{H}_{P}\right)=\mathrm{H}_{P^{\perp}}$ and $\mathcal{K}\left(\mathrm{H}_{P^{\perp}}\right)=\mathrm{H}_{P}$,

(ii) $\mathcal{K}:(u, v) \mapsto\left(-A^{-1} v, A u\right)$ for every $u \in \mathrm{H}_{P}$ and $v \in \mathrm{H}_{P^{\perp}}$ where the map $A:=\left.\mathcal{K}\right|_{\mathrm{H}_{P}}: \mathrm{H}_{P} \rightarrow \mathrm{H}_{P \perp}$ is $\mathbb{R}$-real isometric and surjective with inverse $A^{-1}=-\left.\mathcal{K}\right|_{\mathrm{H}_{P \perp}}$

(iii) Each complex subspace $\mathrm{H}_{P}$ and $\mathrm{H}_{P^{\perp}}$ is separately invariant under the action of $U$ and the maps $\left.\mathcal{P} \ni g \mapsto U\right|_{H_{P}},\left.\mathcal{P} \ni g \mapsto U\right|_{H_{P}}$ are strongly-continuous irreducible locally-faithful unitary complex representations such that

$$
\left.A U_{g}\right|_{\mathrm{H}_{P}}=\left.U_{g}\right|_{\mathrm{H}_{P \perp}} A \quad \forall g \in \mathcal{P} .
$$

Proof. Since $U_{\mathbb{C}_{j}}$ is reducible as established in Lemma 7.6, there must exist an orthogonal projector $P \in \mathfrak{B}\left(\mathrm{H}_{\mathbb{C}_{j}}\right) \backslash\{0, I\}$ such that $P U_{g}=U_{g} P$ for every $g \in \mathcal{P}$. Interpreting $P$ as an $\mathbb{R}$-linear operator in $\mathfrak{B}\left(\mathrm{H}_{\mathbb{R}}\right)$, define the self-adjoint operator $P_{\mathcal{K}}:=P-\mathcal{K} P \mathcal{K} \in \mathfrak{B}\left(\mathrm{H}_{\mathbb{R}}\right)$, it is easy to prove that this operator commutes with both $\mathcal{J}$ and $\mathcal{K}$, i.e., it is $\mathbb{H}$-linear, and so $P_{\mathcal{K}} \in \mathfrak{B}(\mathrm{H})$. Furthermore $P_{\mathcal{K}} U_{g}=U_{g} P_{\mathcal{K}}$ for all $g \in \mathcal{P}$, because $U_{g}$ is quaternionic linear and therefore commutes with $\mathcal{J}$ and $\mathcal{K}$ and also $P U_{g}=U_{g} P$ by hypothesis. Since $U$ is irreducible and $P_{\mathcal{K}}$ is selfadjoint, Proposition 5.3 implies $P_{\mathcal{K}}=a I$ for some $a \in \mathbb{R}$, which, multiplying by $\mathcal{K}$ on the left, can be restated as $\mathcal{K} P+P \mathcal{K}=a \mathcal{K}$, or $\mathcal{K} P=-P \mathcal{K}+a \mathcal{K}$. Since $P P=P$ this can be rewritten as $\mathcal{K} P=-P \mathcal{K} P+a \mathcal{K} P$. Taking the adjoint on both the sides of this identity produces $-P \mathcal{K}=P \mathcal{K} P-a P \mathcal{K}$. Both identities together yield $[\mathcal{K}, P]=a[\mathcal{K}, P]$. The case $a \neq 1$ is not permitted because it implies $[P, \mathcal{K}]=0$, which, together with $[P, \mathcal{J}]=0$ ( $P$ is complex linear by hypothesis), ensures that $P$ is actually quaternionic linear. Since $U$ is irreducible on $\mathrm{H}$, we would get $P=0, I$, which was excluded a priori. It remains the case $a=1$, that is $P-\mathcal{K} P \mathcal{K}=I$. Since $P \neq 0, I$ is an orthogonal projector on $\mathrm{H}_{\mathbb{C}_{j}}, \mathrm{H}_{\mathbb{C}_{j}}$ can be decomposed into an orthogonal direct sum $\mathrm{H}_{\mathbb{C}_{j}}=\mathrm{H}_{P} \oplus \mathrm{H}_{P^{\perp}}$, where $P^{\perp}:=I-P$ and both complex subspaces are nontrivial. Evidently $\mathcal{J}\left(\mathrm{H}_{P}\right)=\mathrm{H}_{P}$ and $\mathcal{J}\left(\mathrm{H}_{P^{\perp}}\right)=\mathrm{H}_{P^{\perp}}$ because each space are complex subspaces of $\mathcal{H}_{\mathbb{C}_{j}}$ and $\mathcal{J}$ is the complex structure used to construct $\mathcal{H}_{\mathbb{C}_{j}}$ out of $\mathrm{H}$. Let us study the interplay of $\mathcal{K}$ and that decomposition proving (i) and (ii). Multiplying $P-\mathcal{K} P \mathcal{K}=I$ by $\mathcal{K}$ on the left, we have $\mathcal{K} P=(I-P) \mathcal{K}$, while a multiplication on the right yields $P \mathcal{K}=\mathcal{K}(I-P)$. Notice that $\mathcal{K}\left(\mathrm{H}_{P}\right)=\mathcal{K} P\left(\mathrm{H}_{\mathbb{C}_{j}}\right)=P^{\perp} \mathcal{K}\left(\mathrm{H}_{\mathbb{C}_{j}}\right)=P^{\perp}\left(\mathrm{H}_{\mathbb{C}_{j}}\right)=\mathrm{H}_{P^{\perp}}$ and, similarly, $\mathcal{K}\left(\mathrm{H}_{P^{\perp}}\right)=\mathcal{K} P^{\perp}\left(\mathrm{H}_{\mathbb{C}_{j}}\right)=P \mathcal{K}\left(\mathrm{H}_{\mathbb{C}_{j}}\right)=P\left(\mathrm{H}_{\mathbb{C}_{j}}\right)=\mathrm{H}_{P}$. Hence, also exploiting the facts that $\mathcal{K}$ is isometric and that $\mathcal{K}(-\mathcal{K})=I$, the map $A: \mathrm{H}_{P} \rightarrow \mathrm{H}_{P \perp}$ defined as $A:=\left.\mathcal{K}\right|_{\mathrm{H}_{P}}$ is a well defined bijective $\mathbb{R}$-linear isometry with inverse given by the isometry $A^{-1}=-\left.\mathcal{K}\right|_{\mathrm{H}_{P \perp}}$. The action of $\mathcal{K}$ can be written $\mathcal{K}(u, v)=\left(-A^{-1} v, A u\right)$ for every $u \in \mathrm{H}_{P}$ and $v \in \mathrm{H}_{P^{\perp}}$. referring to the direct decomposition $\mathbf{H}_{\mathbb{C}_{j}}=\mathrm{H}_{P} \oplus \mathbf{H}_{P^{\perp}}$ Indeed, if $x \in \mathrm{H}_{\mathbb{C}_{j}}$, then $\mathcal{K} x=K\left(P x+P^{\perp} x\right)=\mathcal{K} P x+\mathcal{K} P^{\perp} x=A(P x)-A^{-1}\left(P^{\perp} x\right)$, where $A(P x) \in \mathrm{H}_{P^{\perp}}$ and $-A^{-1}\left(P^{\perp} x\right) \in \mathrm{H}_{P}$. Let us pass to establish (iii). Notice that $U_{g} P=P U_{g}$ ensures that $U_{g}\left(\mathrm{H}_{P}\right) \subset \mathrm{H}_{P}$ and $U_{g}\left(\mathrm{H}_{P \perp}\right) \subset \mathrm{H}_{P^{\perp}}$. Moreover the restrictions of $U_{g}$ to both the factors are clearly bijective isometries on the respective domains, thanks to the facts that $g$ is arbitrary, every $U_{g}$ is isometric and $U_{g^{-1}} U_{g}=I$, so we can refine the inclusions above and write $U_{g}\left(\mathrm{H}_{P}\right)=\mathrm{H}_{P}$ and $U_{g}\left(\mathrm{H}_{P^{\perp}}\right)=\mathrm{H}_{P^{\perp}}$. Notice that, since 
$U_{g} x=U_{g}\left(P x+P^{\perp} x\right)=U_{g}(P x)+U_{g}\left(P^{\perp} x\right)$, the action of $U$ on $\mathrm{H}_{\mathbb{C}_{j}}=\mathrm{H}_{P} \oplus \mathrm{H}_{P \perp}$ can be written $U_{g}(u, v)=\left(U_{g} u, U_{g} v\right)$ for every $u \in \mathrm{H}_{P}$ and $v \in \mathrm{H}_{P^{\perp}}$. We end up with two representations $\left.g \mapsto U_{g}\right|_{H_{P}}$ and $\left.g \mapsto U_{g}\right|_{H_{P \perp}}$ which are clearly unitary and stronglycontinuous on the respective complex Hilbert spaces. Finally we want to prove (30). Let $(u, v) \in \mathrm{H}_{\mathbb{C}_{j}}$, using the above identities we have

$$
\begin{aligned}
& \mathcal{K} U_{g}(u, v)=\mathcal{K}\left(\left.U_{g}\right|_{\mathrm{H}_{P}} u,\left.U_{g}\right|_{\mathrm{H}_{P \perp}} v\right)=\left(-\left.A^{-1} U_{g}\right|_{\mathrm{H}_{P \perp}} v,\left.A U_{g}\right|_{\mathrm{H}_{P}} u\right) \\
& U_{g} \mathcal{K}(u, v)=U_{g}\left(-A^{-1} v, A u\right)=\left(-\left.U_{g}\right|_{\mathrm{H}_{P}} A^{-1} v,\left.U_{g}\right|_{\mathrm{H}_{P \perp}} A u\right)
\end{aligned}
$$

Since $\mathcal{K} U_{g}=U_{g} \mathcal{K}$ and $(u, v)$ is arbitrary, we get the thesis.

To conclude the proof of (iii), we intend to prove that $\left.\mathcal{P} \ni g \mapsto U_{g}\right|_{\mathrm{H}_{P}}$ and $\mathcal{P} \ni g \mapsto$ $\left.U_{g}\right|_{H_{P \perp}}$ are irreducible representations on the respective complex Hilbert spaces which also are locally faithful. We will establish the first result for $\mathbf{H}_{P}$ only, the other case being analogous. Suppose that $\left.g \mapsto U_{g}\right|_{H_{P}}$ is not irreducible, i.e., there exists a projector $0 \leq Q \leq P$ with $Q \neq 0, P$ such that $\left.Q U_{g}\right|_{H_{P}}=\left.U_{g}\right|_{H_{P}} Q$ for every $g \in \mathcal{P}$. Since we are thinking of $Q$ as a projector defined on the whole $\mathrm{H}_{\mathbb{C}_{j}}$, the found identity can be rephrased as $Q U_{g}=U_{g} Q$. Indeed, on $\mathrm{H}_{P}$ that identity reduces to $\left.Q U_{g}\right|_{\mathrm{H}_{P}}=\left.U_{g}\right|_{\mathrm{H}_{P}} Q$, while both sides of $Q U_{g}=U_{g} Q$ vanish on $\mathrm{H}_{P^{\perp}}$. So we can repeat the analysis carried out so far using $Q$ in place of $P$ and finding $\mathrm{H}=\mathrm{H}_{Q} \oplus \mathrm{H}_{Q^{\perp}}$ with $\mathcal{K}\left(\mathrm{H}_{Q}\right)=\mathrm{H}_{Q^{\perp}}$. However, this result implies $\mathrm{H}_{Q^{\perp}}=\mathcal{K}\left(\mathrm{H}_{Q}\right) \subset \mathcal{K}\left(\mathrm{H}_{P}\right)=\mathrm{H}_{P^{\perp}}$ which can be restated as $Q^{\perp} \leq P^{\perp}$, i.e., $P \leq Q$, which was excluded a priori. We conclude that $\left.g \mapsto U_{g}\right|_{\mathrm{H}_{P}}$ (and also $\left.g \mapsto U_{g}\right|_{\mathrm{H}_{P \perp}}$ ) is irreducible. To conclude, we want eventually to demonstrate that the complex strongly-continuous irreducible representations of unitary operators $\left.\mathcal{P} \ni g \mapsto U_{g}\right|_{\mathrm{H}_{P}}$ and $\left.\mathcal{P} \ni g \mapsto U_{g}\right|_{\mathrm{H}_{P} \perp}$ are also locally faithful because $\mathcal{P} \ni g \mapsto U_{g}$ is. Let $W_{e} \subset \mathcal{P}$ be a neighbourhood of the identity of $\mathcal{P}$ where $\mathcal{P} \ni g \mapsto U_{g}$ is faithful and suppose, for example, that $\left.U_{g}\right|_{\mathrm{H}_{P}}=\left.U_{h}\right|_{\mathrm{H}_{P}}$ for some $g, h \in W_{e}$. Exploiting (30), we get $\left.U_{g}\right|_{\mathrm{H}_{P \perp}}=\left.A U_{g}\right|_{\mathrm{H}_{P}} A^{-1}=\left.A U_{h}\right|_{\mathrm{H}_{P}} A^{-1}=\left.U_{h}\right|_{\mathrm{H}_{P}}$ and so, with obious notation, $U_{g}=\left.\left.U_{g}\right|_{\mathrm{H}_{P}} \oplus U_{g}\right|_{\mathrm{H}_{P \perp}}=\left.\left.U_{h}\right|_{\mathrm{H}_{P}} \oplus U_{h}\right|_{\mathrm{H}_{P \perp}}=U_{h}$. Since $U$ is faithful on $W_{e}$ we have that $g=h$, proving the local faithfulness of the restricted representations.

A subsequent lemma is in order.

Lemma 7.8 Assume the hypotheses of Proposition 7.5 and refer to the orthogonal decomposition $\mathrm{H}=\mathrm{H}_{P} \oplus \mathrm{H}_{P \perp}$ and two strongly-continuous irreducible locally-faithful unitary complex representations $\left.\mathcal{P} \ni g \mapsto U\right|_{\mathrm{H}_{P}},\left.\mathcal{P} \ni g \mapsto U\right|_{\mathrm{H}_{P} \perp}$ as in (iii) of Lemma 7.7. The following facts hold.

(iv) The partial isometry $J_{0}$ of the polar decomposition $\tilde{P}_{0}=J_{0}\left|\tilde{P}_{0}\right|$ of the (H-H-linear) anti-selfadjoint generator $\tilde{P}_{0}$ in $\mathrm{H}$ of time-displacements subgroup satisfies

$$
J_{0}=J_{P} \oplus J_{P^{\perp}}
$$

where $J_{P}$ and $J_{P^{\perp}}$ are the analogous operators for the pair of complex representations $\left.\mathcal{P} \ni g \mapsto U_{g}\right|_{H_{P}}$ and $\left.\mathcal{P} \ni g \mapsto U_{g}\right|_{\mathrm{H}_{P \perp}}$. 
(v) $J_{0}$ is a complex structure in the quaternionic Hilbert space $\mathrm{H}$ commuting with the whole representation $U$ and thus $J_{0} \in \mathfrak{R}_{U}^{\prime}$ in particular.

Proof. We simultaneously prove (iv) and (v). First of all we study the complex representations $\left.\mathcal{P} \ni g \mapsto U\right|_{\mathrm{H}_{P}},\left.\mathcal{P} \ni g \mapsto U\right|_{\mathrm{H}_{P \perp}}$ focussing on their anti-selfadjoint generators and their mass operators $M_{U_{P}}^{2}$ and $M_{U_{P \perp}}^{2}$. Let $\widetilde{P}_{\mu}$ the antiselfadjoint generator of $t \mapsto U_{\exp \left(t \mathbf{p}_{\mu}\right)}$ defined on $\mathrm{H}$. As we know from the version of Stone theorem presented in Theorem 2.13, this is in particular a well-defined linear operator over $\mathbf{H}_{\mathbb{R}}$ and $\mathbf{H}_{\mathbb{C}_{j}}$ and it coincides with the corresponding generators of $t \mapsto U_{\exp \left(t \mathbf{p}_{\mu}\right)}$ when reading the representation on $\mathrm{H}_{\mathbb{R}}$ and $\mathbf{H}_{\mathbb{C}_{j}}$, respectively. Due to Stone theorem, $x \in D\left(\tilde{P}_{\mu}\right)$ if and only if there exists $\left.\frac{d}{d t}\right|_{0} e^{t \tilde{P}_{\mu}} x$. As $e^{t \tilde{P}_{\mu}}(u, v)=\left(e^{t \tilde{P}_{\mu}} u, e^{t \tilde{P}_{\mu}} v\right)$ in view of the orthogonal decomposition into complex subspaces $\mathrm{H} \equiv \mathrm{H}_{\mathbb{C}_{j}}=\mathrm{H}_{P} \oplus \mathrm{H}_{P^{\perp}}$, we conclude for $x=(u, v)$ that

$$
\left.\exists \frac{d}{d t}\right|_{0} e^{t \widetilde{P}_{\mu}} x \in \mathrm{H} \quad \text { if and only if }\left\{\begin{array}{c}
\left.\exists \frac{d}{d t}\right|_{0} e^{t \widetilde{P}_{\mu}} u \in \mathrm{H}_{P} \\
\left.\exists \frac{d}{d t}\right|_{0} e^{t \widetilde{P}_{\mu}} v \in \mathrm{H}_{P \perp} .
\end{array}\right.
$$

As an immediate consequence, $P\left(D\left(\widetilde{P}_{\mu}\right)\right)=D\left(\widetilde{P}_{\mu}\right) \cap \mathrm{H}_{P} \subset D\left(\widetilde{P}_{\mu}\right)$ and $P^{\perp}\left(D\left(\widetilde{P}_{\mu}\right)\right)=$ $D\left(\widetilde{P}_{\mu}\right) \cap \mathrm{H}_{P^{\perp}} \subset D\left(\widetilde{P}_{\mu}\right)$, which in turn yields $D\left(\widetilde{P}_{\mu}\right)=P\left(D\left(\widetilde{P}_{\mu}\right)\right) \oplus P^{\perp}\left(D\left(\widetilde{P}_{\mu}\right)\right)$. The generators of the one-parameter unitary subgroups $\left.e^{t \widetilde{P}_{\mu}}\right|_{H_{P}}$ and $\left.e^{t \widetilde{P}_{\mu}}\right|_{\mathrm{H}_{P \perp}}$ are clearly given by $\left.\widetilde{P}_{\mu}\right|_{P\left(D\left(\widetilde{P}_{\mu}\right)\right)}$ and $\left.\widetilde{P}_{\mu}\right|_{P^{\perp}\left(D\left(\widetilde{P}_{\mu}\right)\right)}$, respectively. From $e^{t P_{\mu}} x=e^{t \widetilde{P}_{\mu}} u+e^{t \widetilde{P}_{\mu}} v$ and the above equivalence we have that $\widetilde{P}_{\mu} x=\widetilde{P}_{\mu} u+\widetilde{P}_{\mu} v$, that is $\widetilde{P}_{\mu}=\left.\left.\widetilde{P}_{\mu}\right|_{P\left(D\left(\widetilde{P}_{\mu}\right)\right)} \oplus \widetilde{P}_{\mu}\right|_{P \perp\left(D\left(\widetilde{P}_{\mu}\right)\right)}$. (In the following remember that, as already observed, $D_{G}^{(U)}=D_{G}^{\left(U_{\mathbb{R}}\right)}=D_{G}^{\left(U_{\mathbb{C}_{j}}\right)}$ and $0 \leq$ $\left.M_{U}^{2}=M_{U_{\mathbb{R}}}^{2}=M_{U_{\mathrm{C}_{j}}}^{2}\right)$. Next consider the Gårding domains defined with respect to the representations $\left.U\right|_{\mathrm{H}_{P}}$ and $U_{\mathrm{H}_{P \perp}}$ (hence subspaces of $\mathrm{H}_{P}$ and $\mathrm{H}_{P \perp}$, respectively): $D_{G}^{\left(U_{P}\right)}$ and $D_{G}^{\left(U_{P \perp}\right)}$. We want to prove that they are both subspaces of $D_{G}^{(U)}$ and that $D_{G}^{(U)}=D_{G}^{\left(U_{P}\right)} \oplus$ $D_{G}^{\left(U_{P \perp}\right)}$ when $D_{G}^{(U)}$ is understood as a complex vector space. The former property easily follows from the direct decomposition $\mathrm{H}_{\mathbb{C}_{j}}=\mathrm{H}_{P} \oplus \mathrm{H}_{P^{\perp}}$. Indeed a direct calculation shows that the definitions of the Garding vectors $x[f]$ within $\mathrm{H}_{P}\left(\mathrm{H}_{P^{\perp}}\right)$ or within $\mathrm{H}_{\mathbb{C}_{j}}$ coincide for $x \in \mathrm{H}_{P}\left(\mathrm{H}_{P^{\perp}}\right)$. The equality $D_{G}^{\left(U_{\mathrm{C}_{j}}\right)}=D_{G}^{(U)}$ concludes the proof of this part. To prove the latter, just notice that if $x[f] \in D_{G}^{(U)}$ with $x=(u, v)$, then $x[f]=u[f]+v[f]$ where $u[f] \in D_{G}^{\left(U_{P}\right)}$ and $v[f] \in D_{G}^{\left(U_{P} \perp\right)}$. So, if we consider the operators $M_{U_{P}}^{2}$ and $M_{U_{P \perp}}^{2}$, defined respectively on $D_{G}^{\left(U_{P}\right)}$ and $D_{G}^{\left(U_{P \perp}\right)}$, using the above-proved decompositions for $\widetilde{P}_{\mu}$ we easily get $M_{U}^{2}=M_{U_{P}}^{2} \oplus M_{U_{P} \perp}^{2}$. Finally, $M_{U}^{2} \geq 0$ easily implies that $M_{U_{P}}^{2} \geq 0$ and $M_{U_{P} \perp}^{2} \geq 0$. Summarizing, we end up with two irreducible locally faithful strongly-continuous unitary complex representations of $\mathcal{P}$ : $U_{P}$ on $\mathrm{H}_{P}$ and $U_{P \perp}$ on $\mathrm{H}_{P^{\perp}}$ such that $M_{U_{P}}^{2} \geq 0$ and $M_{U_{P \perp}}^{2} \geq 0$. The respective one-parameter subgroups of time displacement $\left.t \mapsto U_{\exp \left(t \mathbf{p}_{0}\right)}\right|_{\mathrm{H}_{P}}$ 
and $\left.t \mapsto U_{\exp \left(t \mathbf{p}_{0}\right)}\right|_{\mathrm{H}_{P \perp}}$ have generators $\left.\widetilde{P}_{0}\right|_{P\left(D\left(\widetilde{P}_{0}\right)\right)}$ and $\left.\widetilde{P}_{0}\right|_{P^{\perp}\left(D\left(\widetilde{P}_{0}\right)\right)}$, respectively. For each of them the polar decomposition theorem applies and gives $\left.\widetilde{P}_{0}\right|_{P\left(D\left(\widetilde{P}_{0}\right)\right)}=J_{P} X_{P}$ and $\left.\widetilde{P}_{0}\right|_{P^{\perp}\left(D\left(\widetilde{P}_{0}\right)\right)}=J_{P^{\perp}} X_{P^{\perp}}$. We notice for future convenience that $\widetilde{P}_{0}=\left(J_{P} \oplus J_{P^{\perp}}\right)\left(X_{P} \oplus X_{P^{\perp}}\right)$ is the polar decomposition of $\widetilde{P}_{0}$ in $\mathrm{H}_{\mathbb{C}_{j}}$ as a consequence of the uniqueness property of the polar decomposition, the proof being elementary. We are in a position to apply $(\mathrm{g})$ of Theorem 4.3 in [23] establishing that $J_{P}= \pm\left.\mathcal{J}\right|_{\mathrm{H}_{P}}$ and $J_{P^{\perp}}= \pm\left.\mathcal{J}\right|_{\mathrm{H}_{P \perp}}$. Notice that, trivially, $\left.\mathcal{J}\right|_{\mathrm{H}_{P}}$ and $\left.\mathcal{J}\right|_{\mathrm{H}_{P} \perp}$ are unitary operators and they commute with the restrictions of $U_{g}$ to the respective subspaces. In particular $J_{P} \oplus J_{P^{\perp}}$ commutes with $U_{g}=\left.\left.U_{g}\right|_{\mathrm{H}_{P}} \oplus U_{g}\right|_{\mathrm{H}_{P \perp}}$. From $J_{P}= \pm\left.\mathcal{J}\right|_{\mathrm{H}_{P}}$ and $J_{P^{\perp}}= \pm\left.\mathcal{J}\right|_{\mathrm{H}_{P \perp}}$ we also have that $J_{P} \oplus J_{P^{\perp}}$ is an isometry such that $\left(J_{P} \oplus J_{P^{\perp}}\right)^{2}=-I$ : it is a $(\mathbb{C}$-linear $)$ complex structure on $\mathrm{H}_{\mathbb{C}_{j}}$. Thinking of $\widetilde{P}_{0}$ as the generator of time displacements in $\mathrm{H}$, since the polar decomposition is unique in $\mathrm{H}$ and $\mathrm{H}_{\mathbb{C}_{j}}$ as established in (c) Theorem 2.14, $\widetilde{P}_{0}=\left(J_{P} \oplus J_{P^{\perp}}\right)\left(X_{P} \oplus X_{P^{\perp}}\right)$ is also the polar decomposition of $\widetilde{P}_{0}=J_{0}\left|\widetilde{P}_{0}\right|$ in $\mathrm{H}$. Uniqueness entails $J_{0}=J_{P} \oplus J_{P \perp}$. In particular $J_{P} \oplus J_{P^{\perp}}$ must also be quaternionic linear because $J_{0}$ is. Since $J_{P} \oplus J_{P^{\perp}}$ satisfies $\left(J_{P} \oplus J_{P^{\perp}}\right)\left(J_{P} \oplus J_{P^{\perp}}\right)=-I$ and $\left(J_{P} \oplus J_{P^{\perp}}\right)^{*}=-\left(J_{P} \oplus J_{P^{\perp}}\right)$ and the notions of adjoint in $\mathrm{H}$ and $\mathrm{H}_{\mathbb{C}_{j}}$ coincide, $J_{0}$ is a complex structure in $\mathrm{H}$. Moreover, since $J_{P} \oplus J_{P \perp}$ commutes with $U_{g}=\left.\left.U_{g}\right|_{\mathrm{H}_{P}} \oplus U_{g}\right|_{\mathrm{H}_{P \perp}}, J_{0}$ commutes with the whole representation $U$, i.e., $J_{0} \in\left\{U_{g} \mid g \in \mathcal{P}\right\}^{\prime}=\left\{U_{g} \mid g \in \mathcal{P}\right\}^{\prime \prime \prime}=\mathfrak{R}_{U}^{\prime}$ so that $J_{0} \in \mathfrak{R}_{U}^{\prime}$.

To conclude the proof of the main statement it is sufficient to prove that $J_{0} \in \mathfrak{R}_{U}$. Let $B \in \mathfrak{R}_{U}^{\prime}$, then in particular $B e^{t \widetilde{P}_{0}}=e^{t \widetilde{P}_{0}} B$ for every $t \in \mathbb{R}$. Thanks to Proposition 2.15, we immediately get $B J_{0}=J_{0} B$. This means that $J_{0} \in \mathfrak{R}_{U}^{\prime \prime}=\mathfrak{R}_{U}$.

Remark 7.9 The reader interested in an alternative proof of Proposition 7.5 may consult Theorem 9.2.12 of [28], where the real case (see [23]) and the current quaternionic setting are treated on an equal footing.

\subsection{Structure of quaternionic a WRES and equivalence with a complex WRES}

We are now in a position to state and prove the first main result of this work, establishing in particular that a quaternionic WRES $\mathcal{P} \ni p \mapsto U_{p}$ over the quaternionic Hilbert space $\mathrm{H}$ is completely equivalent to a complex Hilbert space WRES $\left.\mathcal{P} \ni p \mapsto U_{p}\right|_{\mathrm{H}_{J_{0}}}$ on a suitable complex Hilbert space $\mathrm{H}_{J_{0}}$ constructed out of a complex structure $J_{0}$ in $\mathrm{H}$ according to (b) Theorem 6.2. Within this equivalent formulation, everything is in agreement with the thesis of Solèr theorem, the complex structure $J_{0}$ is related to the polar decomposition of the generator of time displacements and, independently from its physical meaning, is unique up its sign and Poincaré invariant, i.e., starting from another initial Minkowski frame to describe the symetries of Poincaré group we would obtain the same equivalence of quaternionic-complex structures. The standard Noether correspondence of selfadjoint generators of continuous symmetries and dynamically conserved quantities cen be stated also for the quaternionic case using $J_{0}$ as imaginary unit. 
Theorem 7.10 Consider a quaternionic Wigner Relativistic Elementary System and adopt definitions (27) and (29) (with respect to a given Minkowski reference frame).

Let $\widetilde{P}_{0}=J_{0}\left|\widetilde{P}_{0}\right|$ be the polar decomposition of the anti selfadjoint generator of the subgroup of temporal displacements. The following facts hold provided $M_{U}^{2} \geq 0$.

(a) $J_{0} \in \mathfrak{R}_{U}$ and $J_{0}$ is a complex structure on $\mathrm{H}$.

(b) $J_{0} \in \mathfrak{R}_{U}^{\prime}$, in particular $J_{0} U_{g}=U_{g} J_{0}$ for all $g \in \mathcal{P}$ and so $J_{0}$ is Poincaré invariant (starting from another Minkowski reference frame to define the generators of the one parameter-subgroups of $\mathcal{P}$ we would obtain the same $\left.J_{0}\right)$.

(c) $J_{0} u(\mathbf{A})=u(\mathbf{A}) J_{0}$ for every $\mathbf{A} \in \mathfrak{g}$ in particular $J_{0}\left(D_{G}^{(U)}\right)=D_{G}^{(U)}$.

(d) If $J_{1}$ is a complex structure on $\mathrm{H}$ such that either $J_{1} \in \mathfrak{R}_{U}^{\prime}$ or $J_{1} u(\mathbf{A})=u(\mathbf{A}) J_{1}$ for every $\mathbf{A} \in \mathfrak{g}$ are valid, then $J_{1}= \pm J_{0}$.

(e) If $\mathbf{A} \in \mathfrak{g}$, then $J_{0} \overline{u(\mathbf{A})}=\overline{u(\mathbf{A})} J_{0}$ and this operator is an observable of the WRES.

(f) $U_{J_{0}}:\left.\mathcal{P} \ni g \mapsto U_{g}\right|_{\mathrm{H}_{J_{0}}}$ is a complex WRES over the complex Hilbert space $\mathrm{H}_{J_{0}}$ and furthermore

1. the von Neumann algebra and lattice of elementary observables associated to this complex WRES are the full $\mathfrak{B}\left(\mathrm{H}_{J_{0}}\right)$ and full $\mathcal{L}\left(\mathrm{H}_{J_{0}}\right)$ respectivly;

2. if $A$ is an observable of the initial quaternionic WRES, then $A_{J_{0}}$ is an observable of the associated complex WRES and the map $A \rightarrow A_{J_{0}}$ is bijective over the full set of densely defined selfadjoint operators in $\mathrm{H}_{J_{0}}$ (the observables of the associated complex WRES) which preserves the (point, continuous) spectra.

(g) $\mathfrak{R}_{U}$ is of quaternionic-complex type $\mathfrak{R}_{U}^{\prime}=\left\{a I+b J_{0} \mid a, b \in \mathbb{R}\right\}$ and, referring to the complex Hilbert space $\mathrm{H}_{J_{0}}$, the restriction map $\left.\mathfrak{R}_{U} \ni A \mapsto A\right|_{\mathrm{H}_{J_{0}}} \in \mathfrak{B}\left(\mathrm{H}_{J_{0}}\right)$ defines

1. a norm-preserving weakly-continuous real unital ${ }^{*}$-algebra isomorphism from $\mathfrak{R}_{U}$ onto the full $\mathfrak{B}\left(\mathrm{H}_{J_{0}}\right)$ which maps $J_{0}$ to $j I$;

2. an isomorphism of orthocomplemented complete lattices from $\mathcal{L}_{\Re_{U}}(\mathrm{H})$ onto the full lattice $\mathcal{L}\left(\mathrm{H}_{J_{0}}\right)$ in agreement with the thesis of Solèr theorem;

3. an isomorphism of $\sigma$-complete Boolean lattices $P^{(A)} \ni P_{E}^{(A)} \mapsto P_{E}^{\left(\left.A\right|_{J_{0}}\right)} \in P^{\left(\left.A\right|_{J_{0}}\right)}$ for every fixed $A$ (not necessarily bounded) observable of the quaternionic WRES, $\left.A\right|_{J_{0}}$ being the corresponding observable of the complex WRES. 
Proof. (a) and (b). Everything but the last statement in (b), immediately arise from Prop 7.5. The last sentence in (b) follows from the fact that the penultimate identity in (28) and the uniqueness of the polar decomposition imply that $\widetilde{P}_{0}^{\prime}=\left(U_{p} J_{0} U_{p}^{-1}\right)\left(U_{p} \widetilde{P}_{0} U_{p}^{-1}\right)$ is the polar decomposition of $\widetilde{P}_{0}^{\prime}=U_{p} \widetilde{P}_{0} U_{p}^{-1}$. However, $U_{p} J_{0}=J_{0} U_{p}$ entails $U_{p} J_{0} U_{p}^{-1}=J_{0}$.

(c) Is a weaker case of Proposition 4.12 (a) and (d) since $U_{p} J_{0}=J_{0} U_{p}$.

(d) Since $U$ is irreducible, $\mathfrak{R}_{U}$ is irreducible and thus $\mathfrak{R}_{U}^{\prime}$ must have one of the three mutally exclusive forms listed in Theorem 6.1. The first case is impossible since $\mathfrak{R}_{U}^{\prime}$ would be made of selfadjoint elements while $0 \neq J_{0} \in \mathfrak{R}_{U}^{\prime}$ is anti selfadjoint. The third case is similary forbidden because $\mathfrak{R}_{U} \cap \mathfrak{R}_{U}^{\prime}$ would be made of selfadjoint elements while $0 \neq J_{0} \in \mathfrak{R}_{U} \cap \mathfrak{R}_{U}^{\prime}$ is anti selfadjoint. We conclude that $\mathfrak{R}_{U}^{\prime}=\{a I+b J \mid a, b \in \mathbb{R}\}$ for some complex structure $J$ determined up to its sign. Since $J_{0} \in \mathfrak{R}_{U}^{\prime}$ is a complex structure it must be $J_{0}= \pm J$ and $\mathfrak{R}_{U}^{\prime}=\left\{a I+b J_{0} \mid a, b \in \mathbb{R}\right\}$. This argument also proves the last statement observing that $J_{1} u(\mathbf{A})=\mathbf{A} J_{1}$ for every $\mathbf{A} \in \mathfrak{g}$ is equivalent to $J_{1} \in\left\{U_{g}\right\}_{g \in \mathcal{P}}^{\prime}=\mathfrak{R}_{U}^{\prime}$ in view of (3) Theorem 4.5.

(e). $J_{0} u(\mathbf{A})=u(\mathbf{A}) J_{0}$ is valid from (c). Taking the closures remembering that $J_{0} \in \mathfrak{B}(\mathbf{H})$, we have $J_{0} \overline{u(\mathbf{A})}=\overline{u(\mathbf{A})} J_{0}$. Since $\overline{u(\mathbf{A})}$ is antiselfadjoint and exploiting $J_{0} \in \mathfrak{B}(\mathbf{H})$, we have $\left(J_{0} \overline{u(\mathbf{A})}\right)^{*}=-\overline{u(\mathbf{A})}\left(-J_{0}\right)=\overline{u(\mathbf{A})} J_{0}=J_{0} \overline{u(\mathbf{A})}$ which is therefore self-adjoint. $J_{0} \overline{u(\mathbf{A})}$ commutes with $J_{0}$ so that its PVM is included in $\mathfrak{R}_{U}$ as wanted.

(f). Due to the already established properties, we only have to prove that $\mathcal{P} \ni g \mapsto$ $\left.U_{g}\right|_{H_{J_{0}}}$ is irreducible and locally faithfull. The former property is an immediate consequence of (a) Proposition 5.6, the latter straightforwardly arises from locally faithfulness of $U$ taking advantage of (a),(b) Prop 3.4. So $\left.\mathcal{P} \ni p \mapsto U_{g}\right|_{H_{J_{0}}}$ is a complex WRES over $\mathrm{H}_{J_{0}}$ as requested. Next, irreducibility and the complex version of Schur's lemma eventually yield $\left\{\left.U_{g}\right|_{H_{J_{0}}} \mid g \in \mathcal{P}\right\}^{\prime \prime}=\mathfrak{B}\left(\mathrm{H}_{J_{0}}\right)$, so that, in particular $\mathcal{L}_{\mathfrak{R}_{U_{J_{0}}}}\left(\mathrm{H}_{J_{0}}\right)=\mathcal{L}\left(\mathrm{H}_{J_{0}}\right)$. The last statement immediately arises from Proposition 3.4 (a)(b) and the statement at the end of (c) therein, and Prop 3.5 (d).

(g). The identity $\mathfrak{R}_{U}^{\prime}=\left\{a I+b J_{0} \mid a, b \in \mathbb{R}\right\}$ was established above proving (d), the rest of the thesis easily arises from Theorem 6.2 and Proposition 3.5 (a).

Corollary 7.11 With the same hypotheses as for Theorem 7.10 the following facts are valid.

(a) For all $\mathbf{M}, \mathbf{N} \in E_{\mathfrak{g}}$ the following facts hold

1. $J_{0}\left(u(\mathbf{M})+J_{0} u(\mathbf{N})\right)=\left(u(\mathbf{M})+J_{0} u(\mathbf{N})\right) J_{0}$ and $J_{0} \overline{u(\mathbf{M})+J_{0} u(\mathbf{N})}=\overline{u(\mathbf{M})+J_{0} u(\mathbf{N})} J_{0}$;

2. $\overline{u(\mathbf{M})+J_{0} u(\mathbf{N})}$ is an observable of the quaternionic WRES if and only if it is selfadjoint.

(b) The identity holds

$$
D_{G}^{\left(U_{J_{0}}\right)}=D_{G}^{(U)} \cap \mathrm{H}_{J}
$$

and for all $\mathbf{M}, \mathbf{N} \in E_{\mathfrak{g}}$, 
1. $u_{J_{0}}(\mathbf{M})=\left.u(\mathbf{M})\right|_{\mathrm{H}_{J_{0}} \cap D_{G}^{(U)}}$ and $u_{J_{0}}(\mathbf{M})$ univocally determines $u(\mathbf{M})$;

2. $\overline{u_{J_{0}}(\mathbf{M})+j u_{J_{0}}(\mathbf{N})}=\left.\overline{u(\mathbf{M})+J_{0} u(\mathbf{N})}\right|_{H_{J_{0}}}$ and the left-hand side is self-adjoint (i.e., an observable of the complex associated WRES) if and only if $\overline{u(\mathbf{M})+J_{0} u(\mathbf{N})}$ is an observable of original quaternionic WRES.

Proof. (a) We can apply Proposition 4.12 (d) since $U_{p} J_{0}=J_{0} U_{p}$ obtaining $J_{0}(u(\mathbf{M})+$ $\left.J_{0} u(\mathbf{N})\right)=\left(u(\mathbf{M})+J_{0} u(\mathbf{N})\right) J_{0}$. Taking the closures remembering that $J_{0} \in \mathfrak{B}(\mathrm{H})$, we have $J_{0} \overline{u(\mathbf{M})+J_{0} u(\mathbf{N})}=\overline{u(\mathbf{M})+J_{0} u(\mathbf{N})} J_{0}$. If $\overline{u(\mathbf{M})+J_{0} u(\mathbf{N})}$ is selfadjoint, then its PVM satisfies the same identity in view of (b) Lemma 2.11. In turn, since $\mathfrak{R}_{U}^{\prime}=\left\{a I+b J_{0} \mid a, b \in\right.$ $\mathbb{R}\}$, the PVM of $\overline{u(\mathbf{M})+J_{0} u(\mathbf{N})}$ belongs to $\mathfrak{R}_{U}^{\prime \prime}=\mathfrak{R}_{U}$ as wanted.

(b) Everything easily follows from (a) above and (c) and (d) in Prop4.12 also noticing that the observables of the complex WRES are all of selfadjoint operators in $\mathrm{H}_{J_{0}}$. The fact that $u_{J_{0}}(\mathbf{M})$ univocally determines $u(\mathbf{M})$ is consequence of (b) in Prop 3.4.

\section{Quaternionic relativistic elementary systems (qRES)}

As done in [23] we pass to present a more precise description of an elementary relativistic system.

\subsection{Elementary systems}

We first focus on the general notion of elementary system without referring to a group of symmetry. Exactly as in [23] we adopt the following general definition whose motivation is the same as presented in Sec. 5.1 of [23].

Definition 8.1 An elementary system is an irreducible von Neumann algebra $\mathfrak{R}$ over a separable quaternionic Hilbert space $\mathrm{H}$.

An elementary system is therefore a quantum system such that $\mathfrak{R}^{\prime}$ does not include non-trivial orthogonal projectors: they could be intepreted as elementary observables of another external system independent from the system represented by the self-adjoint operators in $\mathfrak{R}$. If the center of $\mathcal{L}_{\mathfrak{R}}(\mathrm{H})$ is trivial, a sufficient and quite usual condition for having an elementary system is the existence in $\mathfrak{R}$ of a maximal set of compatible observables as discussed in Sec. 5.1 of [23].

In the hypothesis that the elementary propositions of every quantum system are described by the orthogonal projectors of a quaternionic von Neumann algebra, it is clear that every symmetry of the system can be realized in terms of an automorphism of the lattice of projectors itself. According to the discussion in Sec. 5.2 of [23], a relativistic elementary system is first of all an elementary system supporting a continuous representation of Poincaré group which is irreducible with respect to that representation: there are no orthogonal projectors $P \in \mathfrak{R}$ that are fixed under the representation. Continuity must have

a direct physical meaning. In fact, it is induced by seminorms defined by the states. The 
second idea behind the notion of relativistic elementary system is that the representation of the Poincaré group must determine $\mathfrak{R}$ itself. The idea is to view the representation of Poincaré group as a unitary representation (defined up to generalised phases in the centre of the algebra) and to assume that $\mathfrak{R}$ is the algebra generated by these unitary operators. Thanks to Theorem 6.2 and Gleason Theorem (including the quaternionc version proposed by Varadarajan [40]) and corrected in [24] and following a procedure similar to the analog exploited in [23]. we can completely characterize both the states and the symmetries for such an elementary system.

Proposition 8.2 Let $\mathfrak{R}$ be an elementary system over the separable quaternionic Hilbert space $\mathrm{H}$, then the following facts hold true.

(a) Assuming $\operatorname{dim} \mathrm{H} \neq 2$, if $\mu: \mathcal{L}_{\mathfrak{R}}(\mathrm{H}) \rightarrow[0,1]$ is a state (i.e., a $\sigma$-additive probability measure), then there exists a unique positive, selfadjoint unit-trace, trace-class operator $M \in \mathfrak{R}$ such that (see (3) in Sect. 1.4)

$$
\mu(P)=\operatorname{tr}(P M P) \text { for every } P \in \mathcal{L}_{\mathfrak{R}}(\mathrm{H})
$$

Moreover every positive, selfadjoint unit-trace, trace-class operator of $M \in \mathfrak{R}$ defines a state by means of the same relation.

(b) If $h: \mathcal{L}_{\mathfrak{R}}(\mathrm{H}) \rightarrow \mathcal{L}_{\mathfrak{R}}(\mathrm{H})$ is a symmetry of the system (i.e. an automorphism of $\sigma$ complete orthocomplemented lattices), then there exists a $\mathbb{R}$-linear surjective normpreserving map $\mathrm{U}: \mathrm{H} \rightarrow \mathrm{H}$ such that

$$
h(P)=U P U^{-1} \quad \text { for every } P \in \mathcal{L}_{\mathfrak{R}}(\mathrm{H})
$$

and the following further facts are valid.

(i) In both the quaternionic-real and quaternionic-quaternionic cases, $U \in \mathfrak{R}$.

(ii) In the quaternionic-complex case we have two mutually exclusive possibilties

- $U$ belongs to $U \in \mathfrak{R}$,

- $U$ is antilinear w.r.t. $j$ and $k$-thus it is linear with respect to $i$ - and anticommutes with $J$. In this case $U \notin \Re$ but $U^{2} \in \mathfrak{R}$.

(iii) Every real-linear surjective norm-preserving map $V: \mathrm{H} \rightarrow \mathrm{H}$ that satisfies (i) or (ii) depending on the case, satisfies (33) in place of $U$ for the given $h$ if and only if $U^{-1} V \in \mathfrak{R} \cap \mathfrak{R}^{\prime}$.

Finally, every real-linear bijective norm-preserving map $U$ that satisfies (i) or (ii) depending on the case, defines a symmetry by means of (33).

Proof. We need to prove a preliminary technical lemma.

Lemma 8.3 Referring to Theorems 6.1 and 6.2, let $\mathfrak{R}$ be of quaternionic-complex or quaternionic-quaternionic type. Then $A \in \mathfrak{R}$ is trace-class if and only if $A_{J}$, respectively $A_{J K}$, is trace-class. Moreover $\operatorname{tr}\left(A_{J}\right)_{\mathrm{H}_{J}}=\operatorname{tr}(A)_{\mathrm{H}}$ and $\operatorname{tr}\left(A_{J}\right)_{\mathrm{H}_{J K}}=\operatorname{tr}(A)_{\mathrm{H}}$. 
Proof. We study the quaternionic-complex case, the remaining one being strictly analogous. Focusing on $|A|:=\sqrt{A^{*} A}$, proposition 3.5 guarantees that $|A|_{J}=\left(\sqrt{A^{*} A}\right)_{J}=$ $\sqrt{\left(A^{*} A\right)_{J}}=\sqrt{A_{J}^{*} A_{J}}=\left|A_{J}\right|$. If $\left\{f_{k}\right\}_{k \in \mathbb{N}}$ is a Hilbert basis for $\mathrm{H}_{J}$, it is also a Hilbert basis also for $\mathrm{H}(\mathrm{d})$ in view of Prop 3.3 and we have

$$
0 \leq \sum_{k=0}^{\infty}\left\langle f_{k}|| A_{J} \mid f_{k}\right\rangle=\sum_{k=0}^{\infty}\left\langle\left. f_{k}|| A\right|_{J} f_{k}\right\rangle=\sum_{k=0}^{\infty}\left\langle f_{k}|| A \mid f_{k}\right\rangle \leq+\infty,
$$

which immediately implies the first implication of the first statement. Now, suppose that $A$ is trace-class on $\mathrm{H}$, then there exists a Hilbert basis $\left\{e_{k}\right\}_{k \in \mathbb{N}}$ such that $\sum_{k=0}^{\infty}\left\langle e_{k}|| A \mid e_{k}\right\rangle<$ $\infty$. Once this is valid for one basis, it holds true for any Hilbert basis, in particular for any basis of $\mathrm{H}_{J}$, thanks to (d) in view of Prop 3.3. Using (34) again we get the opposite implication. The second property straightforwardly follows from the already exploited fact that every Hilbert basis for $\mathrm{H}_{J}$ is also a Hilbert basis of $\mathrm{H}$ and the observation that the Hermitian scalar product of $\mathrm{H}_{J}$ is the restriction of the one of $\mathrm{H}$.

Let us come back to the main thesis.

(a) Consider a state $\mu: \mathcal{L}_{\mathfrak{R}}(\mathrm{H}) \rightarrow[0,1]$. The thesis is true when $\mathfrak{R}$ is of quaternionicreal type since $\mathcal{L}_{\mathfrak{R}}(\mathrm{H})=\mathcal{L}(\mathrm{H})$ for Theorem 6.2 and we can apply Gleason Theorem as stated in (3) of Section 1.4. Let us pass to the quaternionic-complex case, the only we will consider, the quaternionic-real case being strictly similar using the real version of Gleason theorem. Thanks to Theorem 6.2, the map $\mu$ can be reinterpreted as a $\sigma$-additive probability measure $\mu_{J}$ on the whole $\mathcal{L}\left(\mathrm{H}_{J}\right)$ such that $\mu_{J}\left(P_{J}\right):=\mu(P)$. Since we are dealing with the full lattice of orthogonal projectors of $\mathrm{H}_{J}$, the complex version of Gleason theorem (which is valid for real, complex and quaternionic Hilbert spaces see [40]]) guarantees the existence of a positive, selfadjoint, unit-trace, trace-class operator $T_{J}$ on $\mathrm{H}_{J}$ such that $\mu_{J}\left(P_{J}\right)=\operatorname{tr}\left(T_{J} P_{J}\right)_{\mathrm{H}_{J}}$ for every $P_{J} \in \mathcal{L}\left(\mathrm{H}_{J}\right)$. Thanks to Lemma 8.3, the extension $T$ of $T_{J}$, which belongs to $\mathfrak{R}$ thanks to the identification of the latter with $\mathfrak{B}\left(\mathrm{H}_{J}\right)$, is still of traceclass and unit-trace. Moreover it is clearly positive and self-adjoint thanks to Proposition 3.5. So, let $P \in \mathcal{L}_{\mathfrak{R}}(\mathrm{H})$. The operator $T P$ is still of trace-class and commutes with $J$, hence $(T P)_{J}=T_{J} P_{J}$. Finally $\operatorname{tr}(T P)_{\mathrm{H}}=\operatorname{tr}\left((T P)_{J}\right)_{\mathrm{H}_{J}}=\operatorname{tr}\left(\left(T_{J} P_{J}\right)_{\mathrm{H}_{J}}=\mu_{J}\left(P_{J}\right)=\mu(P)\right.$. Conversely, if $T \in \mathfrak{R}$ is positive, selfadjoint, unit-trace, trace-class operator, the map $\mu(P):=\operatorname{tr}(T P)$ for $P \in \mathcal{L}_{\mathfrak{R}}(\mathrm{H})$ is a well-defined $\sigma$-continuous probability measure as the reader can immediately prove.

(b) Let us prove the initial statement together with (i) and (ii). With the same argument exploited to prove (a), taking the identifications on $\mathfrak{R}$ as stated in Theorem 6.2 into account, we can reinterpret $h$ as a lattice automorphism on $\mathcal{L}(\mathrm{H}), \mathcal{L}\left(\mathrm{H}_{J}\right)$ or $\mathcal{L}\left(\mathrm{H}_{J K}\right)$, respectively. We will denote it as $h, h_{J}$ and $h_{J K}$, respectively. We are in a position to apply Theorems 4.27 and 4.28 in [40]. Sticking to the quaternionic-real and quaternionicquaternionic cases there always exists a unitary operator $U_{0} \in \mathfrak{B}(\mathrm{H})$ or $\mathfrak{B}\left(\mathrm{H}_{J K}\right)$, respectively, such that $h(P)=U_{0} P U_{0}^{-1}$ or $h_{J K}\left(P_{J K}\right)=U_{0} P_{J K} U_{0}^{-1}$ for every orthogonal projector. In the quaternionic-complex case, instead, there exists a either unitary or antiunitary operator $U_{0}$ on $\mathrm{H}_{J}$ such that $h_{J}\left(P_{J}\right)=U_{0} P_{J} U_{0}^{-1}$. 
In the quaternionic-real case $U_{0} \in \mathfrak{R}$, it being $\mathfrak{R}=\mathfrak{B}(\mathrm{H})$ and thus $U:=U_{0}$ is the wanted operator. In the quaternionic-quaternionic case, $U_{0}$ can uniquely be extended to a unitary operator $U \in \mathfrak{R}$ thanks to the isomorphism of $\mathfrak{R}$ and $\mathfrak{B}\left(\mathrm{H}_{J K}\right)$ proved to exist in (c) Theorem 6.2. Since $h_{J K}\left(P_{J K}\right)=h(P)_{J K}$, it is easy to see that $h(P)=U P U^{-1}$ which proves that $U$ is the operator we searched for. In the quaternionic-complex case, when $U_{0}$ is unitary, reasoning as in the quaternionic-quaternionic case, we find a uniquely defined unitary extension $U \in \mathfrak{R}$ of $U_{0}$ such that $h(P)=U P U^{-1}$ so that $U$ is the operator we were looking for. Eventually suppose that, instead, $U_{0}$ is antiunitary, hence a surjective, norm-preserving antilinear map on $\mathrm{H}_{J}$. Decompose every $x \in \mathrm{H}$ as $x=x_{1}+x_{2} k \in \mathrm{H}$ for $x_{1}, x_{2} \in \mathrm{H}_{J}$ and define $U x:=U_{0} x_{1}-U_{0} x_{2} k$. We want to prove that $U$ is the operator we are looking for. By direct inspection one sees that $U$ is real-linear, norm-preserving and surjective, with inverse $U^{-1} x=U_{0}^{-1} x_{1}-\left(U_{0}^{-1} x_{2}\right) k$. Moreover $U(x j)=U\left(x_{1} j-\left(x_{2} j\right) k\right)=$ $U_{0}\left(x_{1} j\right)+U_{0}\left(x_{2} j\right) k=-\left(U_{0} x_{1}\right) j+\left(U_{0} x_{2}\right) k j=-\left[U_{0} x_{1}-\left(U_{0} x_{2}\right) k\right] j=-(U x) j$, hence $U$ is antilinear with respect to $j$. Similarly we find $U(x k)=U\left(-x_{2}+x_{1} k\right)=-U_{0} x_{2}-\left(U_{0} x_{1}\right) k=$ $-\left[U_{0} x_{1}-\left(U_{0} x_{2}\right) k\right] k=-(U x) k$. As a consequence we also have $U(x j k)=(U x) j k$. The same properties can be established for $U^{-1}$. Let us now prove that $U$ anticommutes with $J$. Let $x \in \mathrm{H}$ as above, then $U J x=U\left(x_{1} j+\left(x_{2} j\right) k\right)=U_{0}\left(x_{1} j\right)-\left(U_{0}\left(x_{2} j\right)\right) k=$ $-\left(U_{0} x_{1}\right) j+\left(U_{0} x_{2}\right) j k=-J\left(U_{0} x_{1}\right)+\left(J\left(U_{0} x_{2}\right)\right) k=-J\left(U_{0} x_{1}-\left(U_{0} x_{2}\right) k\right)=-J U x$. Next notice that $U U x=U_{0}^{2} x_{1}+\left(U_{0}^{2} x_{2}\right) k$. Since $U_{0}^{2} \in \mathfrak{B}\left(\mathrm{H}_{J}\right)$ it can be uniquely extended to a unitary operator $W \in \mathfrak{R}$ and by construction it holds $U^{2}=W$ as wanted. Finally, $U$ implements $h$ as required. Indeed, take $P \in \mathcal{L}_{\mathfrak{R}}(\mathrm{H})$, then by definition it follows

$$
\begin{aligned}
& U P U^{-1} x=U_{0} P_{J} U_{0}^{-1} x_{1}+\left(U_{0} P_{J} U_{0}^{-1} x_{2}\right) k=h(P)_{J} x_{1}+\left(h(P)_{J} x_{2}\right) k= \\
& =\widetilde{h(P)_{J}} x=h(P) x
\end{aligned}
$$

Proof of (i) and (ii) are completed.

Let us prove (iii). Suppose first that we are in the quaternionic-real or quaternionicquaternionic case and let $V \in \mathfrak{R}$ such that $V P V^{-1}=U P U^{-1}$ for every projector $P \in$ $\mathcal{L}_{\mathfrak{R}}(\mathrm{H})$. This can be rewritten as $\left(U^{-1} V\right) P\left(U^{-1} V\right)^{-1}=P$ for every $P$. In the quaternionicreal case, Theorem 4.27 in [40] and the $\mathbb{H}$-linearity of $U V^{-1}$ guarantee that $U^{-1} V=a I$ for some $a \in \mathbb{R}$ and the proof of (iii) ends here for this case. In the quaternionicquaternionic case, since both $U^{-1}$ and $V$ commutes with $J, K\left(U^{-1}=U^{*} \in \mathfrak{R}\right)$ we have $\left(U^{-1} V\right)_{J K} P_{J K}\left(U^{-1} V\right)_{J K}^{-1}=P_{J K}$ for every $P_{J K} \in \mathrm{H}_{J K}$. Again, Theorem 4.27 in [40] assures that $\left(U^{-1} V\right)_{J K}=a I_{J K}$ for some $a \in \mathbb{R}$. Uniqueness of the extension from $\mathrm{H}_{J K}$ to $\mathrm{H}$ leads to $U^{-1} V=a I$ for some $a \in \mathbb{R}$ concluding the proof of (ii) in this case, too. Let us pass to the remaining quaternionic-complex case. If $U, V \in \mathfrak{R}$, then we can repeat the argument exploited for the quaternionic-quaternionic case finding $U^{-1} V=a I+b J$ for some $a, b \in \mathbb{R}$ concluding the proof. Suppose conversely that $U, V$ are instead real-linear norm-preserving surjective maps which are antilinear w.r.t $j$ and $k$ and anticommute with $J$. These properties hold also for $U^{-1}$ as a direct calculations shows. The function $U^{-1} V$ turns easily out to be a linear bounded operator which commutes with $J$, hence we can consider $\left(U^{-1} V\right)_{J} \in \mathfrak{B}\left(\mathrm{H}_{J}\right)$. Moreover, since $\left(U^{-1} V\right)_{J} P_{J}\left(U^{-1} V\right)_{J}^{-1}=P_{J}$, Theorem 4.27 
in [40] guarantees that $\left(U^{-1} V\right)_{J}=c I_{J}$ for some $c \in \mathbb{C}$. Again uniqueness of the extension from $\mathrm{H}_{J}$ to $\mathrm{H}$ yields $U^{-1} V=a I+b J$ for some $a, b \in \mathbb{R}$.

Let us pass to the final statement. It is easy to prove in the cases (i) and (ii) with $U \in \mathfrak{R}$. So we only focus attention on the quaternionic-complex case with $U$ which is real-linear, norm-preserving surjective, antilinear with respect to $j$ and $k$ and anticommutes with $J$. Notice that the same properties hold true for $U^{-1}$. The only difficult step is to establish that $h(P):=U P U^{-1}$ is an orthogonal projector of $\mathfrak{R}$ if $P \in \mathcal{L}_{\mathfrak{R}}(\mathrm{H})$. The operator $h(P)$ is clearly quaternionic linear, belongs to $\mathfrak{B}(\mathrm{H})$, and is idempotent. Moreover it commutes with $J$, hence $h(P) \in \mathfrak{R}^{\prime \prime}=\mathfrak{R}$ for every $P \in \mathcal{L}_{\mathfrak{R}}(\mathrm{H})$ because $\mathfrak{R}^{\prime}=\{a I+b J \mid a, b \in \mathbb{R}\}$. To establish that $h(P) \in \mathcal{L}_{\mathfrak{R}}(\mathrm{H})$ it remains to prove that $h(P)$ is selfadjoint. To prove it, let us initially restrict ourselves to $\mathrm{H}_{J}$. Since $U$ anticommutes with $J$ and it is antilinear with respect to $j$, if $x \in \mathrm{H}_{J}$ it must hold $J U x=-U J x=-U(x j)=(U x) j$. As the same holds for $U^{-1}$ we immediately see that $U$ is a well-defined antilinear norm-preserving surjective operator on $\mathrm{H}_{J}$. The standard complex polarization identity implies $\langle U x \mid U y\rangle=\langle y \mid x\rangle$ for every $x, y \in \mathrm{H}_{J}$. The same property is valid for $U^{-1}$. Hence for $x, y \in \mathrm{H}_{J}$, using the established property and selfadjointness of $P$,

$$
\left\langle x \mid U P U^{-1} y\right\rangle=\left\langle P U^{-1} y \mid U^{-1} x\right\rangle=\left\langle U^{-1} y \mid P U^{-1} x\right\rangle=\left\langle U P U^{-1} x \mid y\right\rangle,
$$

so that $\left(\left(U P U^{-1}\right)_{J}\right)^{*}=\left(U P U^{-1}\right)_{J}$. This identity lifts to the whole space $\mathbf{H}$ in view of Proposition 3.4 , giving $h(P)^{*}=h(P)$ and thus $h(P) \in \mathcal{L}_{\mathfrak{R}}(\mathrm{H})$ as required.

\subsection{Relativistic elementary systems in quaternionic Hilbert spaces}

We are now in a position to state the general definition of quaternionic elementary relativistic system that, exactly as the analogous definition in [23] for real and complex systems, includes the notion of elementary system, the presence of an irreducible continuous representation of Poincaré group and the requirement that them representaion itself defines the algebra of observables of the system.

Definition 8.4 A quaternionic relativistic elementary system (qRES) is a couple $(\mathfrak{R}, h)$ where $\mathfrak{R}$ is an irreducible von Neumann algebra over the quaternionic Hilbert space $\mathrm{H}$ and $h: \mathcal{P} \ni g \mapsto h_{g} \in \operatorname{Aut}\left(\mathcal{L}_{\mathfrak{R}}(\mathrm{H})\right)$ is a locally faithful representation of the Poincaré group satisfying the following requirements

(a) $h$ is irreducible, in the sense that $h_{g}(P)=P$ for all $g \in \mathcal{P}$ implies either $P=0$ or $P=I$

(b) $h$ is continuous, in the sense that the map $\mathcal{P} \ni g \mapsto \mu\left(h_{g}(P)\right)$ is continuous for every fixed $P \in \mathcal{L}_{\mathfrak{R}}(\mathrm{H})$ and quantum state $\mu$

(c) $h$ defines the observables of the system. That is, accordingly to Proposition 8.2 and representing $h$ in terms of unitary operators $U_{g} \in \mathfrak{R}$ (defined up to unitary factors in the center $\left.\mathfrak{Z}_{\mathfrak{R}}:=\mathfrak{R} \cap \mathfrak{R}^{\prime}\right), h_{g}(P)=U_{g} P U_{g}^{-1}$ for $g \in \mathcal{P}$ and $P \in \mathcal{L}_{\mathfrak{R}}(\mathrm{H})$, it must be

$$
\left(\left\{U_{g} \mid g \in \mathcal{P}\right\} \cup \mathfrak{Z}_{\mathfrak{R}}\right)^{\prime \prime} \supset \mathcal{L}_{\mathfrak{R}}(\mathrm{H})
$$




\section{Remark 8.5}

(1) Local faithfulness requirement of $h: \mathcal{P} \ni g \mapsto h_{g} \in \operatorname{Aut}\left(\mathcal{L}_{\mathfrak{R}}(\mathrm{H})\right)$ is equivalent to a physically more meaningful requirement:

The representation of spacetime translations subgroup $\mathbb{R}^{4} \ni t \mapsto h_{(I, t)}$ is not trivial. This equivalence immediately arises from Proposition 7.2 when equipping $\operatorname{Aut}\left(\mathcal{L}_{\mathfrak{R}}(\mathrm{H})\right)$ with the topology induced by all off the seminorms $p_{P, \mu}$ for every $P \in \mathcal{L}_{\mathfrak{R}}(\mathrm{H})$ and every state $\mu$ on $\mathcal{L}_{\mathfrak{R}}(\mathrm{H})$, defined as $p_{P, \mu}(h):=|\mu(h(P))|$, since the resulting topological group is Hausdorff as the reader may prove easily. As a byproduct of Proposition 7.2 , if $h$ is not injective, this is only due to the sign of the $S L(2, \mathbb{C})$ part of its argument.

(2) In (c), we have explicitly assumed that $U_{g} \in \mathfrak{R}$ is always valid, excluding the case of $U_{g}$ antilinear w.r.t $j, k$ and anticommuting with $J$ in the quaternionic-complex case (corresponding to an anti unitary operator in the complex Hilbert space $\mathrm{H}_{J}$ ). The reason is the following (the analogous disussion in [23] contained a trivial mistake we correct here). From the polar decomposition of $S L(2, \mathbb{C})$ one sees that every $g \in \mathcal{P}=S L(2, \mathbb{C}) \ltimes \mathbb{R}^{4}$ can be always decomposed into a product of this kind $g=t$ tbbrr where $r$ is a spatial rotation, $b$ a boost, and $t$ a four-translation, hence the real-linear surjective norm-preserving operator $U_{g}=U_{t}^{2} U_{r}^{2} U_{b}^{2}$ generates the symmetry $h_{g}$. It is now clear that, whether or not $U_{t}, U_{r}$ and $U_{b}$ are $\mathbb{H}$-linear and commute with $J$, their squares are $\mathbb{H}$-linear and commute with $J$, and so $U_{g}$ would be linear and commutes with $J$ in every case.

The map $\mathcal{P} \ni g \mapsto U_{g}$ introduced in Definition 8.4 (c) is not, in general, a group representation since we may have $U_{g} U_{h}=\Omega(g, h) U_{g h}$ for operators $\Omega(g, h) \in \mathfrak{U}\left(\mathfrak{Z}_{\mathfrak{R}}\right)$ where $\mathfrak{U}\left(\mathfrak{Z}_{\mathfrak{R}}\right)$ henceforth denotes the set of unitary operators in the center of $\mathfrak{R}$. In particular $U_{e}=\Omega(e, e)$ putting $g=h=e$ in the identity above. Such a map $\mathcal{P} \ni g \mapsto U_{g}$ is known as a projective unitary representation of $\mathcal{P}$, while the function $\Omega: \mathcal{P} \times \mathcal{P} \rightarrow \mathfrak{U}\left(\mathfrak{Z}_{\mathfrak{R}}\right)$ is said to be the multiplier function of the representation.

Remark 8.6 The structure of $\mathfrak{Z}_{\mathfrak{R}}$ implies the following algebraic identifications for a qRES. We have that $\mathfrak{U}\left(\mathfrak{Z}_{\mathfrak{R}}\right)=\mathbb{Z}_{2} I$ - the multiplicators are signs - in the quaternionic-real and quaternionic-quaternionic cases and $\mathfrak{U}\left(\mathfrak{Z}_{\mathfrak{R}}\right)=U(1) I$ - the multiplicators are complex phases - in the quaternionic-complex case.

The associativity property of the operator multiplication easily gives the cocycle-property,

$$
\Omega(r, s) \Omega(r s, t)=\Omega(r, s t) \Omega(s, t) \quad \text { for all } r, s, t \in \mathcal{P} .
$$

For any function $\chi: \mathcal{P} \rightarrow \mathfrak{R}\left(\mathfrak{Z}_{\mathfrak{R}}\right)$ the map $\mathcal{P} \ni g \mapsto \chi(g) U_{g}$ is still a projective representation associated with the same representation $h$ of $\mathcal{P} \ni g \mapsto U_{g}$, whose multiplier is now given by

$$
\Omega_{\chi}(g, h)=\chi(g) \chi(h) \chi(g h)^{-1} \Omega(g, h) \quad \text { for all } g, h \in \mathcal{P} .
$$

A natural question then concerns the possibility of getting rid of the multipliers by finding a function $\chi$ such that $\Omega_{\chi}=I$ in order to end up with a proper unitary representation from a given projective unitary representation. A positive answer can be given for all of the three cases. 
Proposition 8.7 Let $\mathfrak{R}$ and $h$ respectively be the von Neumann algebra and the Poincaré representation of a qRES as in Definition 8.4. The following facts hold.

(a) There exists a locally faithful strongly-continuous unitary representation $\mathcal{P} \ni g \mapsto$ $U_{g} \in \mathfrak{R}$ on $\mathrm{H}$ such that $h_{g}(P)=U_{g} P U_{g}^{-1}$ for every $g \in \mathcal{P}$ and every $P \in \mathcal{L}_{\mathfrak{R}}(\mathrm{H})$.

(b) $\mathcal{P} \ni g \mapsto U_{g} \in \mathfrak{R}$ is irreducible if understood respectively on $\mathrm{H}, \mathrm{H}_{J}$ or $\mathrm{H}_{J K}$ according to the three cases of Theorem 6.2.

Proof. We simultaneously prove (a) and (b). We already know that $h_{g}(\cdot)=V_{g} \cdot V_{g}^{*}$ for some unitary operator $V_{g} \in \mathfrak{R}$. By the continuity hypothesis on $h_{g}$ and (a) of Proposition 8.2. reinterpreting everything within the corresponding space as established by Theorem 6.2, we see that the map

$$
\mathcal{P} \ni g \mapsto \operatorname{tr}\left(P_{\psi} h_{g}\left(P_{\phi}\right)\right)=\left|\left\langle\psi \mid V_{g} \phi\right\rangle\right|^{2} \text { for every }\left\{\begin{array}{l}
\psi, \phi \in \mathrm{H} \text { if } \mathfrak{R}^{\prime} \equiv \mathbb{R} \\
\psi, \phi \in \mathrm{H}_{J} \text { if } \mathfrak{R}^{\prime} \equiv \mathbb{C} \\
\psi, \phi \in \mathrm{H}_{J K} \text { if } \mathfrak{R}^{\prime} \equiv \mathbb{H}
\end{array}\right.
$$

is continuous, where $P_{\chi} \in \mathfrak{R}$ is the projector obtained by lifting to $\mathrm{H}$ the projector on the one-dimensional subspace generated by the generic vector $\chi$ within the space $\mathrm{H}, \mathrm{H}_{J}$ or $\mathrm{H}_{J K}$, depending on the case (the equality in the equation derives from the last statement of Lemma 8.3). Let us focus on the quaternionic-complex case first. Thanks to the above remark, following the analysis contained in the well-known paper [?], we get a stronglycontinuous unitary representation $\mathcal{P} \ni g \mapsto U_{g}$ on $\mathrm{H}_{J}$ such that $U_{g}=\chi_{g}\left(V_{g}\right)_{J}$ for some $\chi_{g} \in U(1)$, hence generating $h_{J}$. More precisely $U_{g}$ can be uniquely extended to a unique unitary operator $\tilde{U}_{g} \in \mathfrak{R}$ such that $\tilde{U}_{g}=\left(a_{g} I+b_{g} J\right) V_{g}$. The map $g \mapsto \tilde{U}_{g}$ generates $h$ and is still faithful as $h$ is. Notice that since $\left\|U_{g} x-x\right\|^{2}=\left\|U_{g} x_{1}-x_{1}\right\|^{2}+\left\|U_{g} x_{2}-x_{2}\right\|^{2}$ the representation $\mathcal{P} \ni g \mapsto \tilde{U}_{g}$ is also strongly continuous on $\mathrm{H}$. Irreducibility of $U$ on $\mathrm{H}_{J}$ follows from the following argument. Since the family $\mathfrak{U}:=\left\{U_{g}, \mid g \in \mathcal{P}\right\}$ is closed under Hermitian conjugation, thanks to Remark 5.2 we need only to prove that $\left(\mathfrak{U}_{J}\right)^{\prime} \cap \mathcal{L}\left(\mathrm{H}_{J}\right)=$ $\left\{0, I_{J}\right\}$, but this is a direct consequence of the irreducibility of $h$. Indeed, if $P_{J}$ is a complex projector commuting with every $U_{g}$ then $\left(h_{g}\right)_{J}\left(P_{J}\right)=U_{g} P_{J} U_{g}^{*}=P_{J}$ for every $g \in \mathcal{P}$ and thus $P=0$ or $P=I$. Let us next focus on the quaternionic-real case. Thanks to the analysis of [8] we can always find a strongly-continuous unitary representation $\mathcal{P} \ni g \mapsto U_{g}$ on $\mathrm{H}$ such that $U_{g}=\chi_{g} V_{g}$ for some $\chi_{g} \in \mathbb{Z}_{2}$, hence generating $h$. Again, it is straightforward to prove the irreducibiity and faithfulness of this representation on $\mathrm{H}$. Let us conclude the proof discussing the quaternionic-quaternionic case. We affirm that we may always choose a representative $\mathcal{P} \ni g \mapsto U_{g}$ on $\mathrm{H}_{J K}$ equivalent to $g \mapsto\left(V_{g}\right)_{J K}$ such that $U_{e}=I_{J K}$, it is strongly continuous over an open neighbourhood of the identity $A_{e}$ and its multiplier $(g, h) \mapsto \Omega(g, h)$ is continuous over $A_{e}^{\prime} \times A_{e}^{\prime}$ with $A_{e}^{\prime} \subset A_{e}$, a smaller open neighbourhood of $e$ which can always be assumed to be connected ( $\mathcal{P}$ is a Lie group and as such it is locally connected). The proof of this fact can be found within the proof of Proposition 12.38 in [22] which is valid both for complex and real Hilbert spaces since 
there is no distinctive role played by the imaginary unit. Since $\Omega(g, h) \in\{ \pm I\}$ which is not connected if equipped with the topology induced by $\mathbb{R}$ and $\Omega(e, e)=I$, the continuity of $\Omega$ guarantees that $\Omega(g, h)=I$ for every $g, h \in A_{e}^{\prime}$. In other words $U_{g} U_{h}=U_{g h}$ for every $g, h \in A_{e}^{\prime}$. As the group $\mathfrak{U}\left(\mathrm{H}_{J K}\right)$ of all unitary operators over $\mathrm{H}_{J K}$ is a topological group with respect to the strong operator topology, the function $\mathcal{P} \ni g \mapsto U_{g}$ is then a local topological-group homomorphism as in Definition B, Chapter 8, Par.47 of [30]. Since, as established in [31], $\mathfrak{U}\left(\mathrm{H}_{J K}\right)$ is connected if $\operatorname{dim} \mathrm{H}_{J K}$ is not finite and $\mathcal{P}$ is a simply connected Lie group, we can apply Theorem 63 30] proving that there exists a strongly-continuous unitary representation $\mathcal{P} \ni g \mapsto W_{g} \in \mathfrak{U}\left(\mathrm{H}_{J K}\right)$ such that $W_{g}=U_{g}$ on some open neighborhood of the identity $A_{e}^{\prime \prime} \subset A_{e}^{\prime}$. If $\operatorname{dim}(\mathrm{H})=n<+\infty$, then $\mathfrak{U}\left(\mathrm{H}_{J K}\right)$ can be identified with the topological group $O(n)$. Its open subgroup $S O(n)$ is the connected component including the identity element $I$. In this situation, we can restrict ourselves to deal with a smaller initial open set $A_{e}^{\prime} \cap B$ where $B$ is the pre-image through the map $U$ (which is continuous on $A_{e}^{\prime}$ ) of an open set including $I$ and completely included in $S O(n)$. As $S O(n)$ is connected, we can finally exploit the same procedure as in the infinite dimensional case, proving that there exists a strongly-continuous unitary representation $\mathcal{P} \ni g \mapsto W_{g} \in \mathfrak{U}\left(\mathrm{H}_{J K}\right)$ such that $W_{g}=U_{g}$ on some open neighbourhood of the identity element $A_{e}^{\prime \prime} \subset A_{e}^{\prime} \cap B$. To conclude, we observe that since the Lie group $\mathcal{P}$ is connected, a standard result guarantees that every $g \in \mathcal{P}$ can be written as $g=g_{1} \cdots g_{n}$ for some $g_{1}, \ldots, g_{n} \in A_{e}^{\prime \prime}$. So, $W_{g}=W_{g_{1}} \cdots W_{g_{n}}=U_{g_{1}} \cdots U_{g_{n}}$ and $h_{g}=h_{g_{1}} \circ \cdots \circ h_{g_{n}}$, from which, dealing with the extensions $\widetilde{W}_{g} \in \mathfrak{R}$, it easily follows $h_{g}=\widetilde{W} \cdot \widetilde{W}_{g}^{*}$ for every $g \in \mathcal{P}$. Again, faithfulness and irreducibility of the representation $\mathcal{P} \ni g \mapsto \tilde{W}_{g}$ immediately arises form the same properties of $h$.

The following technical lemma is useful in the proof of another main result of this work.

Lemma 8.8 Let $\mathrm{H}$ be an either real Hilbert space or quaternionic Hilbert space, with dimension striclty greater than one if quaternionic, then $\mathcal{L}(\mathrm{H})^{\prime}=\mathbb{R} I$. In particular $\mathcal{L}(\mathrm{H})^{\prime \prime}=\mathfrak{B}(\mathrm{H})$ and $\mathfrak{B}(\mathrm{H})^{\prime}=\mathbb{R} I$.

Proof. $\mathcal{L}(\mathrm{H})^{\prime}=\mathbb{R} I$ was established in [23] Lemma 5.16 for $\mathrm{H}$ quaternionic and in [23] Lemma 5.13 for $\mathrm{H}$ real. The final statements immediately arise from $\mathcal{L}(\mathrm{H})^{\prime}=\mathbb{R} I$.

\subsection{Reduction to the complex Hilbert space case}

We are ready to state and prove the second main result of this work.

Theorem 8.9 Let $(\mathfrak{R}, h)$ be a qRES on a quaternionic Hilbert space $\mathrm{H}$. Let $\mathcal{P} \ni g \mapsto$ $U_{g} \in \mathfrak{R}$ be a corresponding locally faithful strongly-continuous unitary representation of $\mathcal{P}$ on $\mathrm{H}$ generating $h$ as in Proposition 8.7.

If the associated mass operator $M_{U}^{2}$ satisfies $M_{U}^{2} \geq 0$, then the following facts are valid.

(a) $\mathfrak{R}$ turns out to be of quaternionic-complex type with commutant generated by the complex structure $J$. 
(b) $U$ results to be irreducible and $\mathfrak{R}=\mathfrak{R}_{U}$ defining a quanternionic WRES with $M_{U}^{2} \geq 0$.

As a consequence of Theorem 7.10, the quaternionic relativistic elementary system can be equivalently described as a complex Wigner relativistic elementary system in the complex Hilbert space $\mathrm{H}_{J}$. In particular $J$, up to sign, coincides to the Poincaré invariant complex structure arising from the polar decomposition of the subgroup of $U$ of the temporal translations.

Proof. The final statements immediately arise from (b), $M_{U}^{2} \geq 0$ and (a), so we prove (a) and (b).

(a) According to Theorem 6.1, $\mathfrak{R}$ can be of three mutually exclusive types. We prove that the quaternionc-real and the quaternionic-complex types are not possible and thus $\mathfrak{R}$ must be of quaternionic-complex case. Let us start by assuming that $\mathfrak{R}$ is of quaternionicreal type. We affirm that $\mathrm{H}$ cannot have dimension 1. If it were the case, suppose for simplicity that $\mathrm{H}=\mathbb{H}$, the representation $U$ could be seen as a strongly-continuous locally faithful unitary representation on the 2-dimensional complex Hilbert space $\mathbf{H}_{\mathbb{C}}=\mathbb{C}^{2}$. Thus $U$ would include a strongly-continuous locally faithful unitary representation $V$ of $S L(2, \mathbb{C})$ on the 2-dimensional complex Hilbert space $\mathbb{C}^{2}$. This is not possible since the continuous finite-dimensional unitary representations of $S L(2, \mathbb{C})$ are completely reducible and the irreducible ones are the trivial representations only [18]. In other words, the initial representation $U$ would be the trivial representation against the local faithfulness hypothesis. So suppose that $\mathrm{H}$ has dimension $>1$. We have that $g \mapsto U_{g}$ is irreducible on $\mathrm{H}$ and $\mathfrak{R}=\mathfrak{B}(\mathrm{H})$, in particular $\mathcal{L}_{\mathfrak{R}}(\mathrm{H})=\mathcal{L}(\mathrm{H})$. Notice that $\mathfrak{Z}_{\mathfrak{R}}=\mathbb{R} I$, hence the physical assumption on the observables of the system reduces to $\mathcal{L}(\mathrm{H}) \subset\left\{U_{g} \mid g \in \mathcal{P}\right\}^{\prime \prime}$. Lemma 8.8 guarantees that $\left\{U_{g} \mid g \in \mathcal{P}\right\}^{\prime \prime}=\mathfrak{B}(\mathrm{H})$. Since $U$ is irreducible, Theorem 7.5 applies, ensuring the existence of a unitary antiselfadjoint operator $J_{0}$ that commutes with the whole representation. So, we have $J_{0} \in\left\{U_{g} \mid g \in \mathcal{P}\right\}^{\prime}=\mathfrak{B}(\mathrm{H})^{\prime}=\mathbb{R} I$, which is clearly impossible because $J_{0}$ is not selfadjoint and not vanishing. Let us prove that $\mathfrak{R}$ of quaternionic-quaternionic type is similarly impossible. We know by Proposition 8.7 that $g \mapsto U_{g}$ is irreducible if understood on $\mathrm{H}_{J K}$ and that $\mathfrak{R}=\mathfrak{B}\left(\mathrm{H}_{J K}\right)$ under the action of the restriction map, in particular $\mathcal{L}_{\mathfrak{R}}(\mathrm{H})$ is isomorphic to $\mathcal{L}\left(\mathrm{H}_{J K}\right)$ under the action of the restriction map. Again, $\mathfrak{Z}_{\mathfrak{R}}=\mathbb{R} I$ so that the assumption $\mathcal{L}_{\mathfrak{R}}(\mathrm{H}) \subset\left(\left\{U_{g} \mid g \in\right.\right.$ $\left.\mathcal{P}\} \cup \mathfrak{Z}_{\mathfrak{R}}\right)^{\prime \prime}$ simplifies to $\mathcal{L}_{\mathfrak{R}}(\mathrm{H}) \subset\left\{U_{g} \mid g \in \mathcal{P}\right\}^{\prime \prime}$. Thanks to Lemma 4.13, the antiselfadjoint generators of $\mathbb{R} \ni t \mapsto U_{\exp \left(\mathbf{p}_{\mu}\right)}$ commutes with $J, K$ and their restriction to $\mathrm{H}_{J K}$ give the generators of the one-parameter subgroups $t \mapsto\left(U_{\exp \left(t \mathbf{p}_{\mu}\right)}\right)_{J K}$, while Proposition 4.14 ensures that $D_{G}^{\left(U_{J K}\right)}=D_{G}^{(U)} \cap \mathrm{H}_{J K}$. This proves that $M_{U_{J K}}^{2}=\left(M_{U}^{2}\right)_{J K}$. Hence, since the positivity of operators is preserved when moving from $\mathrm{H}$ to $\mathrm{H}_{J K}$, we get $M_{U_{J K}}^{2} \geq 0$. Theorem 4.3 of [23] for real Hilbert spaces applies, ensuring the existence of a real-linear unitary antiselfadjoint operator $J_{0} \in \mathfrak{B}\left(\mathrm{H}_{J K}\right)$ such that $J_{0}\left(U_{g}\right)_{J K}=\left(U_{g}\right)_{J K} J_{0}$ for every $g \in \mathcal{P}$. Thanks to Proposition [3.9, $J_{0}$ uniquely extends to a quaternioniclinear unitary antiselfadjoint operator $\tilde{J}_{0}$ on $\mathrm{H}$ which commutes with $J$ and $K$ and the 
relation $J_{0}\left(U_{g}\right)_{J K}=\left(U_{g}\right)_{J K} J_{0}$ similarly extends to $\tilde{J}_{0} U_{g}=U_{g} \tilde{J}_{0}$ for all $g \in \mathcal{P}$. Hence $\tilde{J}_{0} \in\left\{U_{g} \mid g \in \mathcal{P}\right\}^{\prime} \subset \mathcal{L}_{\mathfrak{R}}(\mathrm{H})^{\prime}$. To conclude, since $\mathcal{L}_{\mathfrak{R}}(\mathrm{H})$ is ismorphic to $\mathcal{L}\left(\mathrm{H}_{J K}\right)$ via the restriction map from $\mathrm{H}$ to $\mathrm{H}_{J K}$ and the commutativity of operators is preserved when restricting operators on $\mathrm{H}$ to $\mathrm{H}_{J K}$ and when extending operators on $\mathrm{H}_{J K}$ to operators on $\mathrm{H}$, we have that $J_{0} \in \mathcal{L}\left(\mathrm{H}_{J K}\right)^{\prime}$. Lemma 8.8 eventually guarantees that $\mathcal{L}\left(\mathrm{H}_{J K}\right)^{\prime}=\mathbb{R} I$, which is clearly impossible because $J_{0}$ is not self-adjoint and not vanishing.

(b) The thesis is true if $U$ is irreducible. Indeed, under this hypothesis Theorem 7.10 implies that there is an up-to-sign unique complex structure $J_{0}$ commuting with $U$ and this is obtained from the polar decomposition of the generator of temporal translations. As $\left\{U_{g} \mid g \in \mathcal{P}\right\} \subset \mathfrak{R}$ in view of (b) Proposition 8.2 and (b) Remark 8.5, and since $\mathfrak{R}^{\prime}=\{a I+b J \mid a, b \in \mathbb{R}\}$ for (a), it must be $J= \pm J_{0}$. We may henceforth assume $J=J_{0}$ redefining $J$ up a sign if necessary. Theorem 7.10 also proves that the action on operators of the restriction map from $\mathrm{H}$ to $\mathrm{H}_{J_{0}}$ makes $\mathfrak{R}_{U}$ isomorphic to $\mathfrak{B}\left(\mathrm{H}_{J_{0}}\right)$. We know form (a) that the same action on operators of the restriction map from $\mathrm{H}$ to $\mathrm{H}_{J}$ makes $\mathfrak{R}$ isomorphic to $\mathfrak{B}\left(\mathrm{H}_{J}\right)$. Since $\mathrm{H}_{J}=\mathrm{H}_{J_{0}}$, so that $\mathfrak{B}\left(\mathrm{H}_{J}\right)=\mathfrak{B}\left(\mathrm{H}_{J_{0}}\right)$, we conclude that $\mathfrak{R}=\mathfrak{R}_{U}$.

To conclude the proof of (b) is enough establishing that $\mathcal{P} \ni g \mapsto U_{g}$ is irreducible. The proof of this fact is identical to the proof of Proposition 5.17 in [23] just replacing realcomplex for quaternionic-complex, interpreting the real Hilbert space $\mathrm{H}$ and the relevant subspaces $\mathrm{H}_{P}, \mathrm{H}_{P}^{\perp}$ appearing therein as our quaternionic Hilbert space and corresponding quaternionic subspaces. Finally, Theorem 4.3 in [23] appearing in the proof of Proposition 5.17 in [23] has to be understood here as Theorem 7.10 .

\section{Conclusions}

This works proves that elementary relativistic quantum systems (generalizing Wigner's original ideas) described in a quaternionic Hilbert space, can be equivalently described in complex Hilbert spaces provided a natural spectral condition is satisfied for the observable corresponding to the squared mass of the system. The final picture removes any redundancy of the theory as every selfadjoint operator in the final complex Hilbert space represents an observable, the complex structure is Poincaré invariant, and the standard relation between continuous symmetries and conserved quantities is restored.

Though this result seems physically important, it is worth stressing that it only concerns a very peculiar notion of physical system corresponding to an elementary relativistic particle. From the mathematical side these systems, in complex formulation, are represented by irreducible von Neumann algebra of observables of type $I$. This restriction excludes physically important systems where other types of von Neumann algebras take place (Quantum Field Theory, finite temperature extended systems) or simply the presence of a gauge group (quarks).

It is not obvious whether or not, referring to a larger class of physical systems necessarily different from elementary relativistic particles, a real or quaternionic formulation of quantum theory may have some advantage. First of all one should construct a theory of 
classification of quaternionic von Neumann algebra analogous to the classical one for complex (and real) algebras focusing on the interplay of the lattice of orthogonal projectors of a von Neumann algebra and the von Neumann algebra itself. Some very elementary steps towards this classification are the double commutant theorem (Theorem 2.3) and Proposition 2.12 we have established in this work.

Another interesting issue deserving a closer investigation is the fact that Poincaré symmetry makes sense only in the absence of gravitation, i.e., in the framework of Special Relativity. This restriction leaves open the possibility that quaternionic formulations cannot be excluded when dealing with quantum field theory in (classical) curved spacetime. All these problems will be investigated elsewhere.

\section{Appendix}

\section{A Proof of some propositions}

Proof of Theorem 2.13. If viewing $t \mapsto U_{t}$ as a one-parameter subgroup of unitary operators in $\mathrm{H}_{\mathbb{R}}$, Stone Theorem for real Hilbert spaces (e.g., see Thm 2.9 in [23]) guarantees the existence of a unique antiself-adjoint operator $A$ in $\mathrm{H}_{\mathbb{R}}$ satisfying both the requirements of (10) with respect to the structure of $\mathrm{H}_{\mathbb{R}}$. If we manage to prove that $A$ commutes with $\mathcal{J}$ and $\mathcal{K}$ we get an antiselfadjoint operator on $\mathrm{H}$ satisfying (10) on $\mathrm{H}$ (notice that the derivative in (10) does not depend on the algebra of scalars of the Hilbert space, as the norms of $\mathrm{H}_{\mathbb{R}}$ and $\mathrm{H}$ coincide). For $x \in D(A)$,

$$
\lim _{t \rightarrow 0} \frac{U_{t} \mathcal{J} x-\mathcal{J} x}{t}=\lim _{t \rightarrow 0} \mathcal{J} \frac{U_{t} x-x}{t}=\mathcal{J} \lim _{t \rightarrow 0} \frac{U_{t} x-x}{t}=\mathcal{J} A x
$$

hence $\mathcal{J} x \in D(A)$ and $A \mathcal{J} x=\mathcal{J} A x$. This means $\mathcal{J} A \subset A \mathcal{J}$ and so $\mathcal{J} A=A \mathcal{J}, \mathcal{J}$ being unitary and antiselfadjoint. The same argument applies to $\mathcal{K}$. $A$ is then a quaternionic linear antiselfadjont operator satisfying (10). Now we can focus on the one-parameter unitary subgroup $\mathbb{R} \ni t \mapsto e^{t A}$ in $\mathrm{H}$. So, let $x, y \in D(A)$, then

$$
\begin{aligned}
& \left.\frac{d}{d t}\right|_{t=0}\left\langle x \mid U_{-t} e^{t A} y\right\rangle=\left.\frac{d}{d t}\right|_{t=0}\left\langle U_{t} x \mid e^{t A} y\right\rangle=\left\langle\left.\frac{d}{d t}\right|_{t=0} U_{t} x \mid y\right\rangle+\left\langle x\left|\frac{d}{d t}\right|_{t=0} e^{t A} y\right\rangle= \\
& =\langle A x \mid y\rangle+\langle x \mid A y\rangle=0
\end{aligned}
$$

This proves that $t \mapsto\left\langle x \mid U_{-t} e^{t A} y\right\rangle$ is constant and thus $\left\langle x \mid U_{-t} e^{t A} y\right\rangle=\langle x \mid y\rangle$. Since $D(A)$ is dense and $U_{-t} e^{t A} \in \mathfrak{B}(\mathrm{H})$, we have $U_{-t} e^{t A}=I$, i.e. $U_{t}=e^{t A}$. Now, suppose that there exists another antiselfadjoint operator $B$ on $\mathrm{H}$ such that $U_{t}=e^{t B}$. Using the discussion above for the one-parameter subgroup of the kind $e^{t C}$, we immediately see that $A=B$. We have also proved, en passant, that $A$ coincides with the generator of $U$ with respect to $\mathrm{H}_{\mathbb{R}}$. With the same argument used for $\mathrm{H}_{\mathbb{R}}$, noticing that $\mathrm{H}_{\mathbb{C}_{j}}=\left(\mathrm{H}_{\mathbb{R}}\right)_{\mathcal{J}}$ and $A$ commutes with $\mathcal{J}$, it is easy to prove that $A$ is also the antiselfadjoint of $U$ intepreted as one-parameter unitary group in $\mathrm{H}_{\mathbb{C}_{j}}$. 
Proof of Theorem 2.14. (a) Let us view $A$ as a $\mathbb{R}$-linear operator in $\mathrm{H}_{\mathbb{R}}$ with $D(A)$ intepreted as a real subspace of $\mathrm{H}_{\mathbb{R}}$. With this interpretation, $A$ is still densely defined closed $\mathbb{R}$-linear operator ((e )Prop 2.1) and the operator $A^{*} A$ with this natural domain is densely defined, positive and self-adjoint on $\mathrm{H}_{\mathbb{R}}$ ((a) Thm 2.18 in [23]). Again, Prop 2.1 guarantees that $A^{*} A$ (with the same domain as in the real case) is a densely defined $\mathbb{H}$-linear positive and selfadjoint operator in $\mathrm{H}$. The proof of (a) is completed.

(b) Still interpreting $A$ as a $\mathbb{R}$-linear operator in $\mathrm{H}_{\mathbb{R}}$, the polar decomposition theorem on real Hilbert spaces (e.g., Thm 2.18 in [23]), yields $A=U P$ for a unique pair of $\mathbb{R}$-linear operators $U, P$ that satisfy properties (i)-(iv) and (1)-(3) in $\mathrm{H}_{\mathbb{R}}$. If we manage to prove that $U, P$ are $\mathbb{H}$-linear these properties will be proved valid also in $\mathbf{H}$. Indeed self-adjointness and positivity are preserved when moving from $\mathrm{H}_{\mathbb{R}}$ to $\mathrm{H}$ giving (ii); the norms of the two involved Hilbert spaces are equal to each other and so point (iii) immediately follows; point (i) and (iv) are just function properties, not depending on the scalar field. Finally, properties (1)-(3) immediately derive from the analogous properties valid in $\mathrm{H}_{\mathbb{R}}$ (In particular, Lemma 2.11 implies that $\sqrt{A^{*} A}$ is the same operator in $\mathrm{H}, \mathrm{H}_{\mathbb{R}}$ and $\mathrm{H}_{\mathbb{C}_{j}}$ ). So, we need to prove that $U$ and $P$ commute with $\mathcal{J}, \mathcal{K}$. Take $\mathcal{J}$ and work in $\mathrm{H}_{\mathbb{R}}$. Since $A$ is $\mathbb{H}$-linear, it commutes with $\mathcal{J}$, hence $A=\mathcal{J}^{*} A \mathcal{J}=\mathcal{J}^{*}(U P) \mathcal{J}=\left(\mathcal{J}^{*} U \mathcal{J}\right)\left(\mathcal{J}^{*} P \mathcal{J}\right)$. Since $\mathcal{J}$ is unitary and anti selfadjoint, we see that $\mathcal{J}^{*} U \mathcal{J}$ and $\mathcal{J}^{*} P \mathcal{J}$ satisfy (i)-(iv) and so, by uniqueness of the polar decomposition in $\mathrm{H}_{\mathbb{R}}$, we have $\mathcal{J}^{*} U \mathcal{J}=U$ and $\mathcal{J}^{*} P \mathcal{J}=P$. The same argument applies to $\mathcal{K}$ proving that $U$ and $P$ are $\mathbb{H}$-linear as wanted. To complete the proof of (b), it is enough to prove that the polar decomposition of $A$ in $\mathrm{H}$ is unique. Suppose $A=V Q$ on $\mathrm{H}$ for some $V, Q$ satisfying (i)-(iv). Using an argument similar to that exploited above, we see that $A=V Q$ is a polar decomposition of $A$ in $\mathrm{H}_{\mathbb{R}}$ and so, by uniqueness, $V=U$ and $Q=P$.

(c) The fact that the polar decompositions of $A$ in $\mathrm{H}$ and $\mathrm{H}_{\mathbb{R}}$ coincide has been just established. A strictly analogous argument proves the same result for the polar decompositions of $A$ in $\mathrm{H}_{\mathbb{R}}$ and $\mathrm{H}_{\mathbb{C}_{j}}$.

Proof of Proposition 3.5. (a) If $J A \subset A J$, Lemma 2.11 implies $P_{E}^{(A)} J=J P_{E}^{(A)}$ for every Borelian $E \subset \mathbb{R}$ and Prop. 3.4 easily implies that $\mathcal{B}(\mathbb{R}) \ni E \mapsto\left(P_{E}^{(A)}\right)_{J} \in \mathfrak{B}\left(\mathrm{H}_{J}\right)$ is a complex PVM. Let $x \in \mathrm{H}$ be generic. Since the Hermitian scalar product of $\mathrm{H}_{J}$ is the restriction of $\langle\cdot \mid \cdot\rangle$ to $\mathrm{H}_{J}$ and the self-adjoint operator $A_{J}$ is the analogous restriction of the self-adjoint operator $A$, Lemma 2.10 guarantees that $\mathcal{B}(\mathbb{R}) \ni E \mapsto\left(P_{E}^{(A)}\right)_{J} \in \mathfrak{B}\left(\mathrm{H}_{J}\right)$ is the PVM associated with $A_{J}$ in $\mathrm{H}_{J}$. The proof of the last statement is now trivial.

(b) If $x \in \mathrm{H}$, using notations as in Lemma 2.10, $\mu_{J x}^{P^{(A)}}(E)=\left\langle J x \mid P_{E}^{(A)} J x\right\rangle=\left\langle x \mid J^{*} P_{E}^{(A)} J x\right\rangle=$ $\left\langle x \mid P_{E}^{(A)} x\right\rangle=\mu_{x}^{P^{(A)}}(E)$. If, in particular, $x \in D(f(A))$, we have $\int_{\mathbb{R}}|f(\lambda)|^{2} d \mu_{J x}^{P^{(A)}}(\lambda)=$ $\int_{\mathbb{R}}|f(\lambda)|^{2} d \mu_{x}^{P^{(A)}}(\lambda)<\infty$, which means $J x \in D(f(A))$ and

$$
\left\langle x \mid J^{*} f(A) J x\right\rangle=\langle J x \mid f(A) J x\rangle=\int_{\mathbb{R}} f d \mu_{J x}^{P^{(A)}}=\int_{\mathbb{R}} f d \mu_{x}^{P^{(A)}}=\langle x \mid f(A) x\rangle .
$$

As a consequence, $\left\langle x \mid\left(J^{*} f(A) J-f(A)\right) x\right\rangle=0$ if $x \in D(f(A)) \subset D(f(A) J)$. Since both $f(A)$ and $J^{*} f(A) J$ are selfadjoint operators, $J^{*} f(A) J-f(A)$ is symmetric and 
so the vanishing of the scalar product ensures that $J^{*} f(A) J=f(A)$ on $D(f(A))$, i.e. $J f(A) \subset f(A) J$. Since $J$ is unitary and antiselfadjoint, then $J f(A)=f(A) J$ as wanted. Let us conclude the proof of (b). As a byproduct of the first part of (b), Prop 3.4 implies that the restriction $f(A)_{J}$ a well-defined self adjoint operator of $\mathrm{H}_{J}$. Let us focus on the operator $f\left(A_{J}\right)$ defined on $\mathrm{H}_{J}$ through the complex spectral Theorem. $x \in D\left(f\left(A_{J}\right)\right)$ iff $\int_{\mathbb{R}}|f(\lambda)|^{2} d \mu_{x}^{P^{\left(A_{J}\right)}}<\infty$. Since for (a) $\mu_{x}^{P^{\left(A_{J}\right)}}=\mu_{x}^{P_{J}^{(A)}}$ when $x \in \mathrm{H}_{J}$, we have that $x \in D\left(f(A)_{J}\right) \subset D(f(A))$ and furthermore

$$
\left\langle x \mid f\left(A_{J}\right) x\right\rangle=\int_{\mathbb{R}} f d \mu_{x}^{P^{\left(A_{J}\right)}}=\int_{\mathbb{R}} f d \mu_{x}^{P_{J}^{(A)}}=\langle x \mid f(A) x\rangle=\left\langle x \mid f(A)_{J} x\right\rangle
$$

therefore $\left\langle x \mid\left(f\left(A_{J}\right)-f(A)_{J}\right) x\right\rangle=0$ for $x \in D\left(f\left(A_{J}\right)\right) \subset D\left(f(A)_{J}\right)_{\text {. As }} f\left(A_{J}\right)-f(A)_{J}$ is symmetric on $D\left(f\left(A_{J}\right)\right)$, it is easy to prove that, $f\left(A_{J}\right)-f(A)_{J}=0$ on $D\left(f\left(A_{J}\right)\right)$, i.e. $f\left(A_{J}\right) \subset f(A)_{J}$. Taking the adjoint we also have $f\left(A_{J}\right)^{*} \supset\left(f(A)_{J}\right)^{*}$. Since both operators are selfadjoint, it must be $f\left(A_{J}\right)=f(A)_{J}$.

(c) From the uniqueness of the polar decomposition for closed operators (see [22] for the complex case) it is easy to see that a selfadjoint operator $X$ is positive iff $X=|X|$. Now, thanks to (b) we have $|A|_{J}=\left(\sqrt{A^{*} A}\right)_{J}=\sqrt{\left(A^{*} A\right)_{J}}=\sqrt{A_{J}^{*} A_{J}}=\left|A_{J}\right|$ which gives $A \geq 0$ iff $A=|A|$ iff $A_{J}=|A|_{J}$ iff $A_{J}=\left|A_{J}\right|$ iff $A_{J} \geq 0$, concluding the proof of (c).

(d) As we know (Theorem 4.8 [11](b)), if $A=A^{*}$ its spherical spectrum is completely included in $\mathbb{R}$ and there is not residual spectrum. Furthermore, as in the complex and real Hilbert space cases, $\sigma_{S}(A)$ is the support of $P^{(A)}$, namely, the complement of the largest open set $O \subset \mathbb{R}$ such that $P_{O}^{(A)}=0$ (Theorem 6.6 (b) [13]). Due to the last statement in (a), this is equivalent to saying that $\sigma_{S}(A)$ is the complement of the largest open set $O \subset \mathbb{R}$ such that $P_{O}^{\left(A_{J}\right)}=0$ so that $\sigma_{S}(A)=\sigma\left(A_{J}\right)$. We also know that, exactly as in the real and complex Hilbert space cases, (Theorem 6.6 (d) [13]) $\lambda \in \sigma_{p S}(A)$ iff $P_{\{\lambda\}}^{(A)} \neq 0$. Due to the last statement in (a), this is equivalent to $P_{\{\lambda\}}^{\left(A_{J}\right)} \neq 0$, which is equivalent to $\lambda \in \sigma_{p}\left(A_{J}\right)$. Since, for self-adjoint operators in quaternionic Hilbert spaces we have $\sigma_{c S}(A)=\sigma_{S}(A) \backslash \sigma_{c S}(A)$, exaclty as in real and complex cases, where $\sigma_{c}\left(A_{J}\right)=\sigma\left(A_{J}\right) \backslash \sigma_{p}\left(A_{J}\right)$, we also have $\sigma_{c S}(A)=\sigma_{c}\left(A_{J}\right)$ This concludes the proof.

Proof of Proposition 3.9. (a). It is sufficient to observe that $J X \subset X J$ and $K X \subset X K$ imply that the vectors of the real subspace $D(X) \cap \mathrm{H}_{J K}$ are mapped to $\mathrm{H}_{J K}$ by $X$ and thus $X_{J K}: D(X) \cap \mathrm{H}_{J K} \rightarrow \mathrm{H}_{J K}$ is a well defined $\mathbb{R}$-linear operator in $\mathrm{H}_{J K}$.

(b). Take $A$ as in the hypothesis, referring to the decomposition of Prop 3.8, consider the quaternionic linear subspace of $\mathrm{H}$ defined as $D(\widetilde{A}):=D(A) \oplus D(A) i \oplus D(A) j \oplus D(A) k$. From (14), $L_{q}(D(\widetilde{A})) \subset D(\widetilde{A})$ and $D(A)=D(\widetilde{A}) \cap \mathrm{H}_{J K}$. Now, if $x=x_{1}+x_{2} i+x_{3} j+x_{4} k \in$ $D(\widetilde{A})$, define

$$
\widetilde{A} x:=A x_{1}+\left(A x_{2}\right) i+\left(A x_{3}\right) j+\left(A x_{4}\right) k
$$

which is a $\mathbb{R}$-linear operator on $\mathrm{H}$ extending $A$ and also a (right) quaternionic linear operator as it can be proved by direct inspection. Finally one easily proves that $J \widetilde{A} \subset$ 
$\widetilde{A} J$ and $K \widetilde{A} \subset \widetilde{A} K$ using the given definition of $\widetilde{A}$. Let us pass to the uniqueness property. Suppose there exists a $\mathbb{H}$-linear operator $B$ in $\mathrm{H}$ which extends $A$ and such that $L_{q}(D(B)) \subset D(B)$ and $D(A)=D(B) \cap \mathrm{H}_{J K}$. From this equality we see that $D(\tilde{A}) \subset D(B)$ as $D(B)$ is a quaternionic linear subspace. Now, take any $x \in D(B)$ and decompose it as $x=x_{1}+x_{2} i+x_{3} j+x_{4} k$ with $x_{r} \in \mathrm{H}_{J K}$ for $r=1,2,3,4$ according to Prop 3.8. So,

$$
\begin{aligned}
D(B) \ni\left(L_{j} x\right) j & =\left(-x_{3}+x_{4} i+x_{1} j-x_{2} k\right) j=-x_{1}+x_{2} i-x_{3} j+x_{4} k= \\
& =\left(-x_{1}-x_{3} j\right)+\left(x_{4}+x_{2} j\right) k
\end{aligned}
$$

Hence, we get

$$
\frac{1}{2}\left(x-\left(L_{j} x\right) j\right)=x_{1}+x_{3} j \in D(B) \text { and }-\frac{1}{2}\left(x+\left(L_{j} x\right) j\right) k=x_{4}+x_{2} j \in D(B)
$$

Similarly,

$$
-\left(L_{k}\left(x_{1}+x_{3} j\right)\right) k=x_{1}-x_{3} j \in D(B) \text { and }-\left(L_{k}\left(x_{4}+x_{2} j\right)\right) k=x_{4}-x_{2} j \in D(B)
$$

Combining (37) and (38) together we can easily see that $x_{r} \in D(A)$ for every $r=1,2,3,4$. As a consequence, $D(B) \subset D(A) \oplus D(A) i \oplus D(A) j \oplus D(A) k=D(\tilde{A})$. Summing up we have $D(B)=D(\tilde{A})$ and $B=\widetilde{A}$ from the $\mathbb{H}$-linearity of $B$.

(c) Let us pass to prove the properties (i)-(viii). (i) holds since $J \widetilde{A} \subset \widetilde{A} J$ and $K \widetilde{A} \subset \widetilde{A} K$ are valid, (14) implies $L_{q} \widetilde{A} \subset \widetilde{A} L_{q}$. Exploiting the properties of $L$ it is straightforward to see that it actually holds $L_{q} \widetilde{A}=\widetilde{A} L_{q}$. (ii) is evidently valid from $D(\tilde{A}):=D(A) \oplus$ $D(A) i \oplus D(A) j \oplus D(A) k$ and (36). (iv) Let $B$ be as in the hypotheses, so $x=x_{1}+$ $x_{2} i+x_{3} j+x_{4} k \in D(\widetilde{A B})$ iff $x_{r} \in D(A B)$ for $r=1,2,3,4$, which is equivalent to saying $x_{r} \in D(B)$ and $B x_{r} \in D(A)$ for $r=1,2,3,4$, which is equivalent to requiring $x \in D(\widetilde{B})$ and $\widetilde{B} x \in D(\widetilde{A})$, which is finally equivalent to saying $x \in D(\widetilde{A} \widetilde{B})$. Therefore $D(\widetilde{A B})=D(\widetilde{A} \widetilde{B})$. Since both $\widetilde{A B}$ and $\widetilde{A} \widetilde{B}$ extend $A B$ to $\mathrm{H}$, commutes with $J$ and $K$ and $D(A B)=D(\widetilde{A B}) \cap \mathrm{H}_{J K}=D(\widetilde{A} \widetilde{B}) \cap \mathrm{H}_{J K}$, uniqueness of such an extension gives $\widetilde{A B}=\widetilde{A} \widetilde{B}$. The proofs of (iii) and (v) are analogous. Let us pass to (vi). Suppose that $D(A)=\mathrm{H}_{J K}$ and let $x \in \mathrm{H}$ be decomposed as $x=x_{1}+x_{2} i+x_{3} j+x_{4} k$ with $x_{r} \in \mathrm{H}_{J K}$ for $r=1,2,3,4$. Exploiting (36) and Prop 3.8 we have $D(\widetilde{A})=\mathrm{H}$ and $\|\widetilde{A}\| \leq\|A\| \leq+\infty$ because

$$
\|\widetilde{A} x\|^{2}=\sum_{n=1}^{4}\left\|A x_{n}\right\|^{2} \leq\|A\|^{2} \sum_{n=1}^{4}\left\|x_{n}\right\|^{2}=\|A\|^{2}\|x\|^{2}
$$

On the other hand, since $\widetilde{A} u=A u$ for $0 \neq u \in \mathrm{H}_{J K} \subset \mathrm{H}$, it must be $\|A\| \leq\|\tilde{A}\| \leq+\infty$ and thus $\|\tilde{A}\|=\|A\| \leq+\infty$. In particular $A \in \mathfrak{B}\left(\mathrm{H}_{J K}\right)$ immediately implies $\widetilde{A} \in \mathfrak{B}(\mathrm{H})$. The converse is trivially true as, if $\widetilde{A} \in \mathfrak{B}(\mathrm{H})$ then $D(A)=\mathfrak{B}(\mathrm{H}) \cap \mathfrak{B}\left(\mathrm{H}_{J K}\right)=\mathfrak{B}\left(\mathrm{H}_{J K}\right)$ and furthermore using the argument above, $A \in \mathfrak{B}\left(\mathrm{H}_{J K}\right)$. The proof of (vii) immediately follows from $D(\tilde{A}):=D(A) \oplus D(A) i \oplus D(A) j \oplus D(A) k$ and (b) in Prop 3.8, Let us pass 
to (viii). Notice that $\widetilde{A^{*}} \subset(\tilde{A})^{*}$. indeed, assume that $x \in D\left(\widetilde{A^{*}}\right)$ and $y \in D(\widetilde{A})$, then it holds with obvious notation

$$
\begin{aligned}
\langle x \mid \widetilde{A} y\rangle & =\sum_{\alpha=1}^{4} \sum_{\beta=1}^{4}\left\langle x_{\alpha} \imath_{\alpha} \mid\left(A y_{\beta}\right) \imath_{\beta}\right\rangle=\sum_{\alpha=1}^{4} \sum_{\beta=1}^{4} \overline{\imath_{\alpha}}\left\langle x_{\alpha} \mid A y_{\beta}\right\rangle \imath_{\beta}= \\
& =\sum_{\alpha=1}^{4} \sum_{\beta=1}^{4} \overline{\imath_{\alpha}}\left\langle A^{*} x_{\alpha} \mid y_{\beta}\right\rangle \iota_{\beta}=\left\langle\widetilde{A^{*}} x \mid y\right\rangle
\end{aligned}
$$

As a consequence $\widetilde{A^{*}} \subset(\tilde{A})^{*}$. Let us prove the converse inclusion $\widetilde{A^{*}} \supset(\tilde{A})^{*}$ to conclude. It is enough establishing that $D\left((\widetilde{A})^{*}\right) \subset D\left(\widetilde{A^{*}}\right)$. To this end, suppose $x \in D\left((\widetilde{A})^{*}\right)$. By definition this means that there exists $z \in \mathrm{H}$ such that $\langle x \mid \widetilde{A} y\rangle=\langle z \mid y\rangle$ for every $y \in D(\widetilde{A})$. Take in particular $y=y_{1}+0 i+0 j+0 k \in D(A) \subset D(\widetilde{A})$, then

$$
\sum_{\alpha=1}^{4} \overline{\imath_{\alpha}}\left\langle x_{\alpha} \mid A y_{1}\right\rangle=\langle x \mid \tilde{A} y\rangle=\langle z \mid y\rangle=\sum_{\alpha=1}^{4} \overline{\imath_{\alpha}}\left\langle z_{\alpha} \mid y_{1}\right\rangle
$$

where $\left\langle x_{\alpha} \mid A y_{1}\right\rangle,\left\langle z_{\alpha} \mid y_{1}\right\rangle \in \mathbb{R}$ since the restriction of $\langle\cdot \mid \cdot\rangle$ to $\mathrm{H}_{J K}$ is real-valued. This implies that $\left\langle x_{\alpha} \mid A y_{1}\right\rangle=\left\langle z_{\alpha} \mid y_{1}\right\rangle$ for every $\alpha$. Since $y_{1} \in D(A)$ is generic this means that $x_{\alpha} \in D\left(A^{*}\right)$ for every $\alpha=1,2,3$, 4, i.e., $x \in D\left(\widetilde{A^{*}}\right)$ as wanted concluding the proof of (viii). We prove (ix) concluding the proof. Suppose that $\widetilde{A}$ is closable but $A$ is not, then there exits $\left\{a_{n}\right\}_{n \in \mathbb{N}} \subset D(A)$ such that $a_{n} \rightarrow 0$ as $n \rightarrow+\infty$ and $A a_{n} \rightarrow y \neq 0$. Since $A \subset \widetilde{A}$, we have $\widetilde{A} a_{n} \rightarrow y \neq 0$, which is impossible. Now, on the contrary, suppose that $A$ is closable and $\widetilde{A}$ is not. It must therefore be $D(\widetilde{A}) \ni x_{n} \rightarrow 0$ and $\widetilde{A} x_{n} \rightarrow z \neq 0$. Proposition 3.8 easily entails $\left(x_{n}\right)_{r} \rightarrow 0$ and $A\left(x_{n}\right)_{r_{0}} \rightarrow z_{r_{0}} \neq 0$ for at least one $r_{0}=1,2,3,4$, which is impossible since $A$ is assumed to be closable. The fact that $\overline{\widetilde{A}}=\widetilde{\bar{A}}$ is now an easy consequence of the definition of $\widetilde{A}$, (b) in Proposition 3.8 and the fact that, for general operators in real Banach spaces, $x \in D(\bar{B})$ if and only if there exists a sequence $D(B) \ni x_{n} \rightarrow x$ such that $B x_{n} \rightarrow y_{x}$ for some $y_{x}$, where $\bar{B} x:=y_{x}$.

The proof of $(\mathrm{d})$ is elementary and follows form the definitions.

\section{Proof of Proposition 4.12 ,}

(a) $J\left(D_{G}^{(U)}\right) \subset D_{G}^{(U)}$ immediately arises from boundedness of $J, J U_{g}=U_{g} J$ and the definition of $D_{G}^{(U)}$. As a consequence $J\left(J\left(D_{G}^{(U)}\right)\right) \subset J\left(D_{G}^{(U)}\right)$ so that $D_{G}^{(U)} \subset J\left(D_{G}^{(U)}\right)$ because $J J=-I$ and $D_{G}^{(U)}$ is a subspace. Summing up, $J\left(D_{G}^{(U)}\right)=D_{G}^{(U)}$.

(b) Since $U_{g} J=J U_{g}$, every $U_{g}$ admits $\mathrm{H}_{J}$ as invariant space giving rise to a unitary operator for Prop 3.4 and (b) is manifestly true.

(c) If $x \in \mathrm{H}_{J}$, define $x(f)$ as the only vector of $\mathrm{H}_{J}$ such that $\langle y \mid x(f)\rangle=\int_{G} f(g)\left\langle y \mid U_{g} x\right\rangle d g$ for all $y \in \mathrm{H}_{J}$. Let $x[f]$ be the only vector of $\mathrm{H}$ such that $\langle z \mid x[f]\rangle=\int_{G} f(g)\left\langle z \mid U_{g} x\right\rangle d g$ for all $z \in \mathrm{H}$. We can decompose $z=z_{1}+z_{2} k$ with $z_{1}, z_{2} \in \mathrm{H}_{J}$ due to (b) Prop 3.3, then

$$
\langle z \mid x(f)\rangle=\left\langle z_{1} \mid x(f)\right\rangle-k\left\langle z_{2} \mid x(f)\right\rangle k=\int_{G} f(g)\left\langle z_{1} \mid U_{g} x\right\rangle d g-k\left(\int_{G} f(g)\left\langle z_{2} \mid U_{g} x\right\rangle d g\right)=
$$




$$
=\int_{G} f(g)\left(\left\langle z_{1} \mid U_{g} x\right\rangle-k\left\langle z_{2} \mid U_{g} x\right\rangle k\right) d g=\int_{G} f(g)\left\langle z \mid U_{g} x\right\rangle d g=\langle z \mid x[f]\rangle .
$$

Since this holds for any $z \in \mathrm{H}$, it must be $x(f)=x[f] . D_{G}^{\left(U_{J}\right)}$ is made of $\mathbb{C}_{j}$ complex linear combinations of vectors $x(f)(=x[f])$. As $D_{G}^{U}$ is closed with respect generic quaternionic linear combinations, $D_{G}^{\left(U_{J}\right)} \subset D_{G}^{(U)}$. In particular $D_{G}^{\left(U_{J}\right)} \subset D_{G}^{(U)} \cap \mathrm{H}_{J}$. Let us prove the converse inclusion. Take $\sum_{\alpha=1}^{n} x_{\alpha}\left[f_{\alpha}\right] c_{\alpha} \in D_{G}^{(U)} \cap \mathrm{H}_{J}$ for some $c_{\alpha} \in \mathbb{H}, x_{\alpha} \in \mathrm{H}$ and realvalued functions $f_{\alpha} \in C_{0}^{\infty}(G)$. Thanks to Remark $4.2 x[f] q=(x q)[f]$ and thus we can henceforth suppose that $c_{k}=1$. In view of (b) Prop 3.3. $x_{\alpha}=u_{\alpha}+v_{\alpha} k$ for some $u_{\alpha}, v_{\alpha} \in$ $\mathrm{H}_{J}$, then it is easy to see that $x_{\alpha}\left[f_{\alpha}\right]=u_{\alpha}\left[f_{\alpha}\right]+v_{\alpha}\left[f_{\alpha}\right] k$, where $u_{\alpha}\left[f_{\alpha}\right], v_{\alpha}\left[f_{\alpha}\right] \in D_{G}^{\left(U_{J}\right)} \subset \mathrm{H}_{J}$ (see discussion above) and so

$$
\sum_{\alpha} x_{\alpha}\left[f_{\alpha}\right]=\sum_{\alpha} u_{\alpha}\left[f_{\alpha}\right]+\left(\sum_{\alpha} v_{\alpha}\left[f_{\alpha}\right]\right) k .
$$

Since $\sum_{\alpha} x_{\alpha}\left[f_{\alpha}\right] \in \mathrm{H}_{J}$ it must be $\sum_{\alpha} v_{\alpha}\left[f_{\alpha}\right]=0$ and $\sum_{\alpha} x_{\alpha}\left[f_{\alpha}\right]=\sum_{\alpha} u_{\alpha}\left[f_{\alpha}\right] \in D_{G}^{\left(U_{J}\right)}$. We have found that $D_{G}^{(U)} \cap \mathrm{H}_{J} \subset D_{G}^{\left(U_{J}\right)}$ concluding the proof of (c). (d) First assume $\mathbf{M}=\mathbf{A} \in \mathfrak{g}$. Consider $u(\mathbf{A})=\left.\tilde{A}\right|_{D_{G}^{(U)}}$ where $\widetilde{A}: D(\tilde{A}) \rightarrow \mathbf{H}$ is the anti-selfadjoint generator of $t \mapsto U_{\exp (t \mathbf{A})}$ on $\mathbf{H}$. Thanks to lemma 4.11 we have two important facts. First, it holds $\widetilde{A} J=J \widetilde{A}$ which, together with $J\left(D_{G}^{(U)}\right)=D_{G}^{(U)}$, yields $u(\mathbf{A}) J=J u(\mathbf{A})$ and so we can consider $u(\mathbf{A})_{J}$ which is clearly given by $u(\mathbf{A})_{J}=\left.\widetilde{A}\right|_{D_{G}^{(U)} \cap \mathrm{H}_{J}}=\left.\widetilde{A}\right|_{D_{G}^{(U J}}$. Second, it ensures that $\widetilde{A}_{J}$ is the generator of $t \mapsto\left(U_{\exp (t \mathbf{A})}\right)_{J}$ on $\mathrm{H}_{J}$, and so, by definition, the map $u_{J}$ is necessarily given by $u_{J}(\mathbf{A})=\left.\widetilde{A}_{J}\right|_{D_{G}^{\left(U_{J}\right)}}=\left.\widetilde{A}\right|_{D_{G}^{\left(U_{J}\right)}}$. Putting the two conclusions together we get the thesis for $\mathbf{M}=\mathbf{A} \in \mathfrak{g}$. The extension to a generic element of $E_{\mathfrak{g}}$ is trivial making use of (22).

\section{Proof of Proposition 7.2 .}

Let us prove that (a) implies (b). Assuming (a) is true, there is a neighborhood of $\{(I, 0)\}$ where $f$ is injective, therefore the map $\mathbb{R}^{4} \ni t \mapsto f((I, t)) \in G$ cannot be the constant function always attaining the neutral element $e$ of $G$, so (b) is valid.

Let us now prove that (b) implies (a). $\operatorname{Ker}(f)$ is a normal subgroup of $S L(2, \mathbb{C}) \ltimes \mathbb{R}^{4}$ and is also closed because $f$ is continuous and, if $e \in G$ is the unit elements, $\{e\}$ is closed because $G$ is Hausdorff. Since $\operatorname{Ker}(f)$ is a closed subgroup of a Lie group, it must be a Lie subgroup due to Cartan theorem. As we prove below, the normal Lie subgroups of $S L(2, \mathbb{C}) \ltimes \mathbb{R}^{4}$ are $(1) S L(2, \mathbb{C}) \ltimes \mathbb{R}^{4}$ itself, $(2)\left\{( \pm I, t) \mid t \in \mathbb{R}^{4}\right\}$ and its subgroups $(3) \mathbb{R}^{4} \equiv\left\{(I, t) \mid t \in \mathbb{R}^{4}\right\}$, (4) $\{( \pm I, 0)\}$, (5) $\{(I, 0)\}$. Since, by hypothesis $\mathbb{R}^{4} \ni t \mapsto f((I, t)) \in G$ is injective, $\operatorname{Ker}(f)$ cannot be as in the cases $(1),(2),(3)$. Only (4) and (5) are possible. In both cases $f$ is injective at least in a neighbourhood of $(I, 0)$ so (a) is valid.

Regarding the last statement in the thesis of Proposition 7.2, observe that holding (b), from the above analysis we conclude that $f$ is not injective if and only if $\operatorname{Ker}(f)=$ 
$\{( \pm I, 0)\}$

To conclude we have to prove that the normal Lie subgroups of $S L(2, \mathbb{C}) \ltimes \mathbb{R}^{4}$ are those mentioned. More precisely (2), (3) and (4), since (1) and (5) are trivial. First one has to identify the connected normal Lie subgroups. They correspond to ideals of the Lie algebra invariant under the adjoint action. In the Lie algebra, the adjoint action on $\mathbb{R}^{4}$ is irreducible and on the quotient we have the adjoint action of the real simple Lie algebra $s l(2, \mathbb{C})$ which is also irreducible. Hence there is only one non-trivial ideal corresponding to the translation group. This shows that closed normal subgroups are either discrete or their identity component consists of the translation group. Let us start with the discrete case. Discrete normal subgroups of connected Lie groups are central. And it is easy to figure out the centre, which is $\{ \pm I\}$ in $S L(2, \mathbb{C})$; so this is the only discrete normal subgroup. If $N$ is a Lie normal subgroup whose identity component consists of translations, then its quotient by $\mathbb{R}^{4}$ is a discrete subgroup of $S L(2, \mathbb{C})$, hence either trivial or the centre. This argument produces precisely 3 non-trivial closed normal subgroups, i.e., (2),(3) and (4) and these are all.

\section{Acknowledgements}

. The authors are grateful to Professor K.-H. Neeb for useful discussions and technical suggestions.

\section{References}

[1] S. L. Adler: Quaternionic Quantum Mechanics and Quantum Fields. International Series of Monographs on Physics, Vol. 88 The Clarendon Press Oxford University Press, New York, (1995)

[2] D. Aerts, B. van Steirteghem: Quantum Axiomatics and a theorem of M.P. Solèr. Int. J. Theor. Phys. 39, 497-502, (2000).

[3] V. Bargmann: On Unitary Ray Representations of Continuous groups. Ann. Math. 59, 1-46 (1954)

[4] E.G., Beltrametti, G. Cassinelli: The logic of quantum mechanics. Encyclopedia of Mathematics and its Applications, vol. 15, Addison-Wesley, Reading, Mass., (1981)

[5] J. Diximier, J. and P. Malliavin, P.: Factorisations de fonctions et de vecteurs indéfiniment différentiables, Bull. Sciences Mathematiques 102 305-330 (1978)

[6] A. Dvurečenskij: Gleason's Theorem and Its Applications, Kluwer Academic Publishers (1993)

[7] K. Engesser, D.M. Gabbay, D. Lehmann (editors): Handbook of Quantum Logic and Quantum Structures. Elsevier, Amsterdam (2009) 
[8] G. Emch, Mécanique quantique quaternionienne et relativité restreinte, Helvetica Physica Acta, 36 (1963)

[9] D. Finkelstein, J. M. Jauch, S. Schiminovich, and D. Speiser: Foundations of Quaternion Quantum Mechanics. J. Math. Phys. 3, 207 (1962)

[10] A.M. Gleason, Measures on the closed subspaces of a Hilbert space. J. Math. Mech. 6(6), 885-893 (1957)

[11] R. Ghiloni, V. Moretti and A. Perotti: Continuous slice functional calculus in quaternionic Hilbert spaces Rev. Math. Phys. 25, 1350006 (2013)

[12] R. Ghiloni, V. Moretti and A. Perotti: Spectral properties of compact normal quaternionic operators, in Hypercomplex Analysis: New perspectives and applications, (Eds S. Bernstein, U. Kaehler, I. Sabadini, F. Sommen), Trends in Mathematics, Birkhauser, Basel (2014)

[13] R. Ghiloni, V. Moretti and A. Perotti: Spectral representations of normal operators via Intertwining Quaternionic Projection Valued Measures

Rev.Math.Phys. 29, 1750034 (2017)

[14] S.S. Holland: Orthomodularity in infinite dimensions; a theorem of M. Solèr. Bulletin of the American Mathematical Society, 32, 205-234, (1995)

[15] R. Kadison, J.R. Ringrose: Fundamentals of the Theory of Operator Algebras, (Vol. I, II, III, IV) Graduate Studies in Mathematics, AMS (1997)

[16] B. Li: Real Operator Algebras. World Scientific (2003)

[17] J.M. Jauch. Projective Representations of the Poincaré Group in a Quaternionic Hilbert Space in Group Theory and Applications, E.M Loeb editor, Academic Press (1968)

[18] A. W. Knapp: Representation Theory of Semisimple Groups. 3rd Printing, Princeton University Press (2001)

[19] G. Mackey: The Mathematical Foundations of Quantum Mechanics. Benjamin, New York (1963)

[20] F. Maeda, S. Maeda: Theory of symmetric lattices. Springer (1970)

[21] R. Meise, D. Vogt: Introduction to Functional Analysis, The Clarendon Press Oxford University Press (1997)

[22] V. Moretti: Spectral Theory and Quantum Mechanics, Mathematical Structure of Quantum Theories, Symmetries and Introduction to the Algebraic Formulation. Springer, 2nd Edition (2018) 
[23] V. Moretti and M. Oppio: Quantum theory in real Hilbert space: How the complex Hilbert space structure emerges from Poincaré symmetry Rev. Math. Phys. 29 1750021 (2017)

[24] V. Moretti and M. Oppio: The correct formulation of Gleason's theorem in quaternionic Hilbert spaces. Ann. Henri Poincaré 19 (2018), 3321-3355

[25] M.A. Najmark, A.I. Stern: Theory of Group Representations. Springer (1982)

[26] E. Nelson: Analytic Vectors. Ann. Math. 70, 572-614 (1959)

[27] K.-H. Neeb, G. Ólafsson: Antiunitary Representations and Modular Theory. arXiv:1704.01336v1 [math.RT] (2017)

[28] M. Oppio: On the Necessity of Complex Numbers in Quantum Mechanics. PhD Thesis in Mathematics, (Advisor V. Moretti, Referees, K.-H. Neeb and N. Pinamonti) Trento University (2018) http://eprints-phd.biblio.unitn.it/2936/

[29] C. Piron: Axiomatique Quantique. Helv. Phys. Acta 37 439-468 (1964)

[30] L. Pontrjagin: Topological Groups. Princeton University Press, Princeton University Press (1946)

[31] C.R. Putnam and A. Wintner, The connectedness of the orthogonal group in Hilbert space. Proc.Nat.Acad.Sci. U.S.A. 37, (1951), 110-122

[32] M. Rédei, Quantum Logic in Algebraic Approach, Kluver, (1998)

[33] W. Rudin, Functional Analysis 2nd edition, Mc Graw Hill, (1991)

[34] K. Schmüdgen, Unbounded Operator Algebras and Representation Theory, Birkhäuser Basel, (1990)

[35] K. Schmüdgen, Unbounded Self-adjoint Operators on Hilbert Space, Springer, 2012

[36] B. Simon: Quantum dynamics: From automorphism to Hamiltonian. Studies in Mathematical Physics, Essays in Honor of Valentine Bargmann (ed. E.H. Lieb, B. Simon and A.S. Wightman), Princeton University Press, Princeton, 327-349 (1976)

[37] M.P. Solèr: Characterization of Hilbert spaces by orthomodular spaces. Communications in Algebra, 23, 219-243 (1995)

[38] K. Urbanik, F.B. Wright, Absolute-valued algebras. Proc. Amer. Math. Soc. 11 (1960), 861-866

[39] V.S. Varadarajan, Lie Groups, Lie Algebras and their Representations, Springer (1984) 
[40] V.S. Varadarajan, Geometry of Quantum Theory. 2nd Edition, Springer (2007)

[41] F.W. Warner, Foundations of differentiable manifolds and Lie groups. Springer, Berlin (1983) 